\title{
STEADY STATES OF ROTATING STARS AND GALAXIES
}

\author{
WALTER A. STRAUSS AND YILUN WU
}

\begin{abstract}
A rotating continuum of particles attracted to each other by gravity may be modeled by the Euler-Poisson system. The existence of solutions is a very classical problem. Here it is proven that a curve of solutions exists, parametrized by the rotation speed, with a fixed mass independent of the speed. The rotation is allowed to vary with the distance to the axis. A special case is when the equation of state is $p=\rho^{\gamma}, 6 / 5<$ $\gamma<2, \gamma \neq 4 / 3$, in contrast to previous variational methods which have required $4 / 3<\gamma$.

The continuum of particles may alternatively be modeled microscopically by the Vlasov-Poisson system. The kinetic density is a prescribed function. We prove an analogous theorem asserting the existence of a curve of solutions with constant mass. In this model the whole range $(6 / 5,2)$ is allowed, including $\gamma=4 / 3$.
\end{abstract}

\section{Contents}

1. Introduction

1.1. Informal statement of results

1.2. Technique and outline

2. The Euler model

2.1. Assumptions

2.2. Main theorem

2.3. Construction

3. Euler model: basic properties

3.1. Properties of the pressure and enthalpy

3.2. Properties of the radial density $\rho_{0}$

3.3. Properties of the dilating function $g_{\zeta}$

4. Euler model: analysis of the linearized operator

4.1. Linearization

4.2. Radial part of the Euler kernel

4.3. Non-radial part of the Euler kernel

4.4. Compactness

4.5. Realization of the mass condition

4.6. Example: oblateness for constant rotation

5. Euler Model: Fréchet differentiability

6. Vlasov model

6.1. Main theorem

6.2. Construction

6.3. Radial solutions

7. Vlasov model: analysis of the linearized operator

7.1. Linearization

7.2. Analysis of the Vlasov kernel

8. Vlasov model: Fréchet differentiability

References 


\section{INTRODUCTION}

We consider a continuum of particles attracted to each other by gravity but subject to no other forces. Initially they are static and spherical but then they begin to rotate after some perturbation and thereby flatten at the poles and expand at the equator. This is a simple model of a rotating star or planet. It can also model a rotating galaxy with its billions of stars. In this paper we consider slow rotations and look for steady states of the resulting configuration. We find a connected set of such states with constant mass.

This is a very classical problem that goes back to MacLaurin, Jacobi, Poincaré, Liapunov et al., who assumed the density of the rotating fluid to be homogeneous or almost homogeneous, which is of course physically unrealistic if we want to consider a rotating gaseous star or a rotating galaxy. See Jardetzky [12 for a nice account of the classical history on this problem. More realistic was the later work of Lichtenstein [14] and Heilig [10, who approached the problem by means of an implicit function theorem (IFT) in function space. They made realistic assumptions on the density but the mass of their solutions changes as the body changes its speed of rotation.

A different approach was begun by Auchmuty and Beals [3] using a variational method (VAR) with a mass constraint. The main difficulty in this approach is to prove that the minimizing solution has compact support. Their approach was generalized by many authors, including Auchmuty 2, Caffarelli and Friedman [4, Friedman and Turkington 8], Li [13, Chanillo and $\mathrm{Li}$ [5, Luo and Smoller [15], Wu [18, and $\mathrm{Wu}$ 19. Compared with IFT, the VAR method has the advantage that the mass is constant. On the other hand, compared with VAR, the IFT method has the advantage that the construction provides a continuous curve of solutions depending on the angular velocity $\omega$ and with obviously compact support of oblate shape.

In this paper we improve the IFT approach by constructing solutions that keep the mass constant, so that there is no loss or gain of particles when the body changes its rotation speed. Also, we allow the angular velocity to be non-uniform, thus including the physically interesting cases of differential rotation into our model.

Our first model, which we call EP (Euler-Poisson), is generalized from Lichtenstein and Heilig. In EP we assume that the particles move inside the body according to the steady compressible Euler equations, subject to internal forces of Newtonian gravity given by the Poisson equation, with a variable speed $\omega$ of rotation around an axis, and an equation of state for the pressure $p=p(\rho)$ where $\rho$ is the density.

Our second model, which we call VP (Vlasov-Poisson), is generalized from Rein [17. In $\mathrm{VP}$ the particles are given by a microscopic density $f(x, v)$ satisfying the Vlasov equation, where the macroscopic density is given by $\rho(x)=\int_{\mathbb{R}^{3}} f(x, v) d v$ as in kinetic theory. The rotation is provided by a rather arbitrary function of the microscopic angular momentum $x_{1} v_{2}-x_{2} v_{1}$. Although the two models are clearly different, they have some similarities.

In this paper we treat both models by constructing a continuous curve of solutions using the IFT approach. The parameter along this curve is the intensity of the rotation speed. As distinguished from all the previous literature using the IFT approach, the mass is constant along the curve, and the angular velocity can be non-uniform.

1.1. Informal statement of results. For the EP model we begin with the steady compressible Euler-Poisson equations for the density $\rho \geq 0$, subject to the internal forces of gravity due to the particles themselves. The speed $\omega(r)$ of rotation around the $x_{3}$-axis is allowed to depend on $r=r(x)=\sqrt{x_{1}^{2}+x_{2}^{2}}$. The inertial forces are entirely due to the rotation. In the region $\left\{\rho_{\kappa}>0\right\}$ the governing equation turns out to be

$$
\rho_{\kappa} * \frac{1}{|x|}+\kappa \int_{0}^{r} \omega^{2}(s) s d s-h\left(\rho_{\kappa}\right)=\text { constant }
$$


Here $\omega(r)$ is a given function, $\kappa$ is a constant measuring the intensity of rotation, and $h$ is defined by $h^{\prime}(\rho)=\frac{p^{\prime}(\rho)}{\rho}$ with $h(0)=0$. The pressure is $p$ and the specific enthalpy is $h$. The constant of gravity is assumed to be 1. Our theorem for the EP model, informally stated in a special case is as follows.

Let the equation of state be the power law $p(\rho)=C \rho^{\gamma}$ where $\frac{6}{5}<\gamma<2$ and $\gamma \neq \frac{4}{3}$. For any mass $M$ of a non-rotating star, there exists $\bar{\kappa}>0$ and a continuous curve $\kappa \mapsto \rho_{\kappa}$ from $(-\bar{\kappa}, \bar{\kappa})$ into $C_{c}^{1}\left(\mathbb{R}^{3}\right)$ such that each $\rho_{\kappa}$ is an axisymmetric solution of (1.1) with total mass $M=\int_{\mathbb{R}^{3}} \rho_{\kappa} d x$.

For the VP model we begin with the steady Vlasov-Poisson system for the microscopic density $f(x, v)$ and the gravitational potential $U(x)$. Extending Rein [17, we look for solutions $f$ that have the form

$$
f_{\kappa}(x, v)=C_{\kappa} \phi(E, L), \quad E=\frac{1}{2}|v|^{2}+U_{\kappa}(x), \quad L=\kappa\left(x_{1} v_{2}-v_{1} x_{2}\right),
$$

where $\phi$ is a prescribed function. The constant $C_{\kappa}$ is chosen so that the total mass $\iint_{\mathbb{R}^{6}} f_{\kappa}(x, v) d v d x$ is a given constant $M$ independent of $\kappa$. Because $x_{1} v_{2}-x_{2} v_{1}$ is the $x_{3}$ component of the angular momentum, $\kappa$ provides the intensity of rotation. The governing equation then takes the form

$$
-\Delta_{x} U_{\kappa}=4 \pi \int_{\mathbb{R}^{3}} f_{\kappa}(x, v) d v .
$$

Since (1.2) determines $f_{\kappa}$ in terms of $U_{\kappa}$, (1.3) is a single equation for $U_{\kappa}$. Our theorem for the VP model, informally stated in a special case, is as follows.

Assume that $\phi(E, L)=(-E)_{+}^{-\mu} \psi(L)$ with $-\frac{7}{2}<\mu<\frac{1}{2}$, and $\psi$ a suitably regular nonnegative function. For any mass $M$ of a non-rotating star, there exists $\bar{\kappa}>0$ and a continuous curve $\kappa \mapsto U_{\kappa}$ from $(-\bar{\kappa}, \bar{\kappa})$ into $C^{3}\left(\mathbb{R}^{3}\right)$ such that each $U_{\kappa}$ provides an axisymmetric solution of (1.3), with total mass $M$.

When this paper was almost complete, we learned of very recent work [1] by Jang and Makino, who also studied the EP model using an IFT approach in the case of the power law $p=C \rho^{\gamma}$ and constant rotation speed. The perturbation they take is very different from the one of this paper. Rather than deforming a given non-rotating star solution as we do, they directly perturb the specific enthalpy in a function space, which appears to be a more general type of perturbation. However, as in Lichtenstein and Heilig's work, their perturbation also does not keep the total mass constant. Their analysis is restricted to the range $\frac{6}{5}<\gamma<\frac{3}{2}$.

1.2. Technique and outline. Following Lichtenstein and his successors, we construct rotating solutions by deforming the corresponding spherically symmetric stationary solution $\rho_{0}$. The deformation $g_{\zeta}$, characterized by a function $\zeta(x)$ that is axisymmetric around the $x_{3}$-axis, even in $x_{3}$, is defined by

$$
g_{\zeta}(x)=\left(1+\frac{\zeta(x)}{|x|^{2}}\right) x .
$$

The factor $1 /|x|^{2}$ is a convenience that differs from the previous authors. For EP we then define $\rho_{\zeta}(y)=\mathcal{M}(\zeta) \rho\left(g_{\zeta}^{-1}(y)\right)$, where $\mathcal{M}(\zeta)$ is chosen to assure the total mass to be independent of $\zeta$. For VP an analogous but slightly more complicated definition is used, again designed to keep the total mass independent of $\zeta$.

Both models are formulated implicitly in the form $\mathcal{F}(\zeta, \kappa)=0 \in X$ for $\zeta$ in a function space $X$ of axisymmetric functions. Here $\mathcal{F}(0,0)=0$ corresponds to the spherically symmetric non-rotating solution and $\kappa$ measures the intensity of rotation, which is assumed to be small. Therefore what must be proven is that $\mathcal{F}$ is differentiable and that $\mathcal{L}=\frac{\partial \mathcal{F}}{\partial \zeta}(0,0)$ is an isomorphism.

Sections 2 and 3 are devoted to a precise formulation of the EP model and the statement of the main EP theorem (Theorem 2.1). In Section 5 we prove the Fréchet differentiability of $\mathcal{F}$. This turns out to be a surprisingly technical task. Our space $X$ has the norm $\|\zeta\|_{X}=\sup _{|x| \leq 1}|\nabla \zeta(x)| /|x|$, which is simpler than the norm used in the previous literature. 
The simplification is aided by our extra factor $1 /|x|^{2}$ in the definition of $g_{\zeta}$. We compute the formal derivative, then show that it maps $X$ to $X$, and finally prove that it is Fréchet differentiable.

In Section 4 we prove for the EP model that the linearized operator $\mathcal{L}=\frac{\partial \mathcal{F}}{\partial \zeta}(0,0)$ is essentially of the form $I+K$, where $K$ is compact. Thus the main task is to prove that the nullspace of $\mathcal{L}$ is trivial. This task is considerably more difficult than the previous studies (Lichtenstein and Heilig) because the mass constraint adds a whole new nonlocal term to $L$ and because the rotation speed $\omega$ depends on $r$, the distance to the rotation axis. There are several novel aspects to the proof. Using a delicate scaling argument, we prove in Theorem 4.1 that the nullspace is indeed trivial, assuming that the mass of a radial solution strictly changes as the density at the origin changes. In the example $p(\rho)=C \rho^{\gamma}$, this assumption corresponds to the condition that $\gamma \neq \frac{4}{3}$. The power $\gamma=\frac{4}{3}$ is the "white dwarf" model, for which the mass is invariant under scaling and for which the nullspace of $\mathcal{L}$ is not trivial. At the end of Section 4, the constant angular velocity case is examined in more detail. Properties of $\mathcal{L}$ imply that the supports of the perturbed solutions are wider at the equator than at the poles, confirming the usual physical intuition of the shape of slowly rotating stars.

The rest of the paper is devoted to the VP model. In Section [ we present the precise formulation of the model, define an operator $\mathcal{F}$ different from the EP model, and state the main theorem (Theorem 6.1). In Section 8 we prove the Fréchet differentiability of $\mathcal{F}$, which is analogous to the previous proof for EP. Section 7 is devoted to the linearized operator $\mathcal{L}=\frac{\partial \mathcal{F}}{\partial \zeta}(0,0)$ for the VP model. The triviality of its nullspace is quite delicate and is significantly different both from that for the EP model and from that in Rein 17 . For the VP model there are no exceptional cases sensitive to mass invariance. The special choice of $\phi(E, L)$ mentioned above with $-\frac{7}{2}<\mu<\frac{1}{2}$ corresponds to $\frac{6}{5}<\gamma<2$, where $\gamma=1+\left(\frac{3}{2}-\mu\right)^{-1}$.

\section{The Euler MOdel}

In this model, the gas is described by the compressible Euler-Poisson equations. The equations in full generality are given as

$$
\left\{\begin{array}{l}
\rho_{t}+\nabla \cdot(\rho v)=0, \\
(\rho v)_{t}+\nabla \cdot(\rho v \otimes v)+\nabla p=\rho \nabla U, \\
U(x, t)=\int_{\mathbb{R}^{3}} \frac{\rho\left(x^{\prime}, t\right)}{\left|x-x^{\prime}\right|} d x^{\prime} .
\end{array}\right.
$$

Here, the first two equations hold where $\rho>0$, and the last equation defines $U$ on the entire $\mathbb{R}^{3}$. To close the system, one prescribes an isentropic equation of state

$$
p=p(\rho) .
$$

To model a rotating star, we look for steady axisymmetric rotating solutions to (2.1), i.e. we assume $\rho=\rho(x)=\rho(A x)$ for any rotation $A$ about the $x_{3}$-axis, $v=\kappa \omega(r)\left(-x_{2}, x_{1}, 0\right)$, where $r=\sqrt{x_{1}^{1}+x_{2}^{2}}$, where the angular velocity distribution $\omega(r)$ is prescribed. With such specifications, the first equation in (2.1) concerning mass conservation is identically satisfied. The second equation in (2.1) concerning momentum conservation can be simplified to

$$
-\rho \kappa \omega^{2}(r) e_{r}+\nabla p=\rho \nabla U, \quad e_{r}=\left(x_{1}, x_{2}, 0\right)
$$

The first term in (2.3) can be written as $-\rho \nabla\left(\int_{0}^{r} \omega^{2}(s) s d s\right)$. If we introduce the specific enthalpy $h$ as

$$
h(\rho)=\int_{0}^{\rho} \frac{p^{\prime}(\alpha)}{\alpha} d \alpha
$$

then (2.3) becomes

$$
\nabla\left(U+\kappa \int_{0}^{r} \omega^{2}(s) s d s-h(\rho)\right)=0
$$


where again

$$
U(x)=\int_{\mathbb{R}^{3}} \frac{\rho\left(x^{\prime}\right)}{\left|x-x^{\prime}\right|} d x^{\prime} .
$$

Thus the Euler model is reduced to (2.5), (2.6). We wish to solve for $\rho$ with prescribed $\omega(r)$ and $p(\rho)$.

2.1. Assumptions. We now state our assumptions on $\omega(r)$ and $p(\rho)$. For $\omega(r)$ we simply assume

$$
\omega^{2}(r) \in C_{l o c}^{1, \beta}[0, \infty) \text { for some } \beta=\beta(\omega) \in(0,1) .
$$

For $p(s)$ we make the following three assumptions.

$$
p(s) \in C^{3}(0, \infty), p^{\prime}>0 .
$$

There exists $\gamma \in(1,2)$ such that,

$$
\lim _{s \rightarrow 0^{+}} s^{3-\gamma} p^{\prime \prime \prime}(s)=-c_{0}<0 .
$$

There exists $\gamma^{*} \in\left(\frac{6}{5}, 2\right)$ such that

$$
\lim _{s \rightarrow \infty} s^{1-\gamma^{*}} p^{\prime}(s)=c_{1}>0 .
$$

Example. All of the above assumptions are satisfied if we let $\omega(r)$ be a constant and take the equation of state to be a power law $p(s)=s^{\gamma}$ for some $\gamma \in\left(\frac{6}{5}, 2\right)$. Indeed, in this case $\gamma^{*}=\gamma=c_{1}, c_{0}=\gamma(\gamma-1)(2-\gamma), h(s)=\frac{\gamma}{\gamma-1} s^{\gamma-1}$.

2.2. Main theorem. The existence of radial (spherically symmetric) solutions is wellknown, as will be discussed in Section 3, and is summarized in the following proposition.

Proposition 2.1. Let $p$ satisfy the assumptions above. For every $R>0$, there exists a solution $\rho_{0}(x)$ of (2.5), (2.6) with the following properties.

- $\rho_{0}$ is radial (with $\omega \equiv 0$ ),

- $\rho_{0}>0$ in $B_{R}=\{|x|<R\}, \rho_{0}=0$ in $\mathbb{R}^{3} \backslash B_{R}$,

- $\rho_{0} \in C^{2}\left(B_{R}\right) \cap C^{1, \alpha}\left(\mathbb{R}^{3}\right)$, where $\alpha=\min \left(\frac{2-\gamma}{\gamma-1}, 1\right)$.

To state a key condition in the main theorem, we need to express the total mass of radial solutions near a given $\rho_{0}$. In Section 4.2 we will show that a unique radial solution $\rho$ to (2.5), (2.6) with $\omega(r)=0$ exists provided the density $\rho(0)$ at the center is sufficiently close to $\rho_{0}(0)$, where $\rho_{0}$ is a solution given in Proposition 2.1. Let $M(\rho(0))=\int_{\mathbb{R}^{3}} \rho d x$ be the total mass of such a solution with center density $\rho(0)$. We also denote the mass of $\rho_{0}$ simply by $M$. Our main theorem regarding non-radial solutions of the EP model is as follows.

Theorem 2.1. Let $\omega$ and $p$ satisfy the assumptions above. Assume

$$
M^{\prime}\left(\rho_{0}(0)\right) \neq 0 \text {. }
$$

Then there exists $\bar{\kappa}>0$ such that, for every $|\kappa|<\bar{\kappa}$, there exists a solution $\rho_{\kappa}$ of (2.5), (2.6) that is

- axisymmetric and even in $x_{3}$;

- $\rho_{\kappa} \geq 0$ and is compactly supported (with support near $B_{R}$ );

- $\rho_{\kappa} \in C^{1, \alpha}\left(\mathbb{R}^{3}\right)$ where $\alpha$ is the same as in Proposition 2.1;

- the mapping $\kappa \mapsto \rho_{\kappa}$ is continuous from $(-\bar{\kappa}, \bar{\kappa})$ to $C_{c}^{1}\left(\mathbb{R}^{3}\right)$;

- $\int_{\mathbb{R}^{3}} \rho_{\kappa} d x=M$, where the constant $M$ is the total mass of $\rho_{0}$.

Remark 1. The prime (') in (2.11) means differentiation. This condition can be interpreted as saying that nearby solutions have genuinely different masses. In fact, we will show that (2.11) is a necessary and sufficient condition for the kernel of a key linearized operator to be trivial. 
Remark 2. $\rho_{\kappa}$ has higher regularity in the interior of its support, but in general is only $C^{1, \alpha}$ up to the boundary of its support.

It is of interest, due to Theorem 2.1, to find conditions on $p$, in addition to the ones given in (2.8), (2.9) and (2.10), for which (2.11) is satisfied. In the following theorem, we give two types of such conditions. One of them concerns power laws $p(s)=s^{\gamma}$, and the other is a general type of condition.

Theorem 2.2. 2.11) is satisfied if either

(a) $p(s)=s^{\gamma}, \gamma \in\left(\frac{6}{5}, 2\right), \gamma \neq \frac{4}{3}$, or

(b) $p(s)$ satisfies (2.8), (2.9) and (2.10), as well as

$$
p^{\prime}(s)<h(s) \leq 2 p^{\prime}(s) \text { for } s>0 .
$$

The proof of Theorem 2.2 is is given in Section 4.5.

Remark 3. By the definition of $h$ given in (2.4), condition (2.12) is positive linear in $p$. It follows that, if $p_{1}(s)$ and $p_{2}(s)$ both satisfy (2.12), then any positive linear combination of them also. As a consequence, (2.11) is satisfied if, for example, $p(s)=s^{\gamma_{1}}+s^{\gamma_{2}}$ with $\gamma_{1}, \gamma_{2} \in\left[\frac{3}{2}, 2\right)$.

Remark 4. The classical results using variational methods started by 3 only include solutions for power laws with $\gamma>\frac{4}{3}$. Theorem 2.1] and 2.2 construct rotating star solutions for power laws with $\gamma \in\left(\frac{6}{5}, \frac{4}{3}\right)$ as well.

2.3. Construction. The solutions $\rho_{\kappa}$ will be perturbations of the radial solution $\rho_{0}$ given in Proposition 2.1, as we shall now describe. The radial solution satisfies

$$
\Delta\left(h\left(\rho_{0}\right)\right)+4 \pi \rho_{0}=0, \quad u_{0}=h\left(\rho_{0}\right)
$$

Since the analysis is identical for any value of the radius $R$, we assume without loss of generality that $R=1$. As stated above, the support of the perturbed solutions will have a single connected component. Thus (2.5) can equivalently be written as

$$
U(x)-U(0)+\kappa \int_{0}^{r(x)} \omega^{2}(s) s d s-h(\rho)(x)+h(\rho)(0)=0 .
$$

Here $\kappa$ is a constant quantifying the smallness of the rotation. Following Lichtenstein and Heilig, we look for solutions $\rho_{\kappa}$ of the form

$$
\rho_{\kappa}(x)=\mathcal{M}(\zeta) \rho_{0}\left(g_{\zeta}^{-1}(x)\right), \quad \zeta=\zeta_{\kappa},
$$

for some axisymmetric function $\zeta: \overline{B_{1}} \rightarrow \mathbb{R}$. The dilating function is

$$
g_{\zeta}(x)=x\left(1+\frac{\zeta(x)}{|x|^{2}}\right)
$$

and the mass factor is

$$
\mathcal{M}(\zeta)=\frac{M}{\int_{B_{1}} \rho_{0}(x) \operatorname{det} D g_{\zeta}(x) d x}=\frac{\int_{B_{1}} \rho_{0}(x) d x}{\int_{B_{1}} \rho_{0}(x) \operatorname{det} D g_{\zeta}(x) d x} .
$$

This means that we are perturbing $\rho_{0}$ by composing with an axisymmetric diffeomorphism $g_{\zeta}^{-1}$, and rescaling the whole function by $\mathcal{M}(\zeta)$ to get a new density distribution which has the same total mass as the unperturbed solution $\rho_{0}$. Such a solution is different from that of Lichtenstein and Heilig in that

(i) the diffeomorphism (2.16) has an $|x|^{2}$ factor on the denominator in the last term;

(ii) the rescaling factor $\mathcal{M}(\zeta)$ is here to keep the total mass unchanged; and

(iii) the function $\omega(r)$ is not necessarily constant, thus allowing differential rotation. 
The difference (i) is technical and will allow us to present a more elegant argument of the whole construction, while (ii) and (iii) are our improvements of the basic physical construction.

Using (2.15), (2.14) becomes

$$
\begin{aligned}
& \mathcal{M}(\zeta) \int_{g_{\zeta}\left(B_{1}\right)} \rho_{0}\left(g_{\zeta}^{-1}\left(y^{\prime}\right)\right)\left(\frac{1}{\left|z-y^{\prime}\right|}-\frac{1}{\left|y^{\prime}\right|}\right) d y^{\prime}+\kappa \int_{0}^{r(z)} \omega^{2}(s) s d s \\
& -h\left(\mathcal{M}(\zeta) \rho_{0}\left(g_{\zeta}^{-1}(z)\right)\right)+h\left(\mathcal{M}(\zeta) \rho_{0}\left(g_{\zeta}^{-1}(0)\right)\right)=0 .
\end{aligned}
$$

(2.18) holds for $z \in g_{\zeta}\left(B_{1}\right)$. Composing the left hand side with $g_{\zeta}$, that is $z=g_{\zeta}(x)$, the equation takes the form

$$
\begin{aligned}
& \mathcal{M}(\zeta) \int_{g_{\zeta}\left(B_{1}\right)} \rho_{0}\left(g_{\zeta}^{-1}\left(y^{\prime}\right)\right)\left(\frac{1}{\left|g_{\zeta}(x)-y^{\prime}\right|}-\frac{1}{\left|y^{\prime}\right|}\right) d y^{\prime}+\kappa \int_{0}^{r\left(g_{\zeta}(x)\right)} \omega^{2}(s) s d s \\
& -h\left(\mathcal{M}(\zeta) \rho_{0}(x)\right)+h\left(\mathcal{M}(\zeta) \rho_{0}(0)\right)=0
\end{aligned}
$$

for $x \in B_{1}$.

Now we rewrite (2.19) in terms of a nonlinear operator $\mathcal{F}$. Let the function $\zeta$ in (2.16) belong to the space

$$
X=C^{1}\left(\overline{B_{1}}\right) \cap\left\{\zeta \mid \zeta(x) \text { is axisymmetric and even in } x_{3}, \zeta(0)=0, \sup _{x \in \dot{B}_{1}} \frac{|\nabla \zeta(x)|}{|x|}<\infty\right\}
$$

endowed with the norm

$$
\|\zeta\|_{X}=\sup _{x \in \dot{B}_{1}} \frac{|\nabla \zeta(x)|}{|x|}
$$

If we define the operator $\mathcal{F}$ as

$$
\begin{aligned}
& \mathcal{F}(\zeta, \kappa)=\mathcal{M}(\zeta) \int_{g_{\zeta}\left(B_{1}\right)} \rho_{0}\left(g_{\zeta}^{-1}\left(y^{\prime}\right)\right)\left(\frac{1}{\left|g_{\zeta}(x)-y^{\prime}\right|}-\frac{1}{\left|y^{\prime}\right|}\right) d y^{\prime}+\kappa \int_{0}^{r\left(g_{\zeta}(x)\right)} \omega^{2}(s) s d s \\
& \quad-h\left(\mathcal{M}(\zeta) \rho_{0}(x)\right)+h\left(\mathcal{M}(\zeta) \rho_{0}(0)\right),
\end{aligned}
$$

then (2.19) is equivalent to

$$
\mathcal{F}(\zeta, \kappa)=0
$$

We will prove that $\mathcal{F}: B_{\epsilon}(X) \times \mathbb{R} \rightarrow X$, where $B_{\epsilon}(X)=\left\{\zeta \in X \mid\|\zeta\|_{X}<\epsilon\right\}$, and $\epsilon$ is suitably small, as will be specified in the following discussion.

\section{EUler MODEL: BASIC PROPERTIES}

In this section, we describe the standard construction of $\rho_{0}$ in Proposition 2.1, and prove a few basic properties of the mapping $g_{\zeta}$ defined in (2.16). As has been explained above, we work with the solutions supported on the unit ball $B_{1} \subset \mathbb{R}^{3}$.

3.1. Properties of the pressure and enthalpy. (2.9) implies

$$
\lim _{s \rightarrow 0^{+}} s^{2-\gamma} p^{\prime \prime}(s)=\frac{c_{0}}{2-\gamma}, \quad \lim _{s \rightarrow 0^{+}} s^{1-\gamma} p^{\prime}(s)=\frac{c_{0}}{(\gamma-1)(2-\gamma)} .
$$

It is equally straightforward to show that

$$
\begin{gathered}
\lim _{s \rightarrow 0^{+}} s^{1-\gamma} h(s)=\frac{c_{0}}{(\gamma-1)^{2}(2-\gamma)}, \quad \lim _{s \rightarrow 0^{+}} s^{2-\gamma} h^{\prime}(s)=\frac{c_{0}}{(\gamma-1)(2-\gamma)}, \\
\lim _{s \rightarrow 0^{+}} s^{3-\gamma} h^{\prime \prime}(s)=-\frac{c_{0}}{\gamma-1}, \quad \lim _{s \rightarrow 0^{+}} s^{4-\gamma} h^{\prime \prime \prime}(s)=c_{0} \frac{\gamma+1}{\gamma-1} . \\
\lim _{s \rightarrow \infty} s^{1-\gamma^{*}} h(s)=\frac{c_{1}}{\gamma^{*}-1} .
\end{gathered}
$$


Since $1<\gamma<2$, (3.1) implies $p^{\prime}(s) / s$ is integrable at 0 , hence $h$ is continuous on $[0, \infty)$. Since $p$ is strictly increasing, $h$ is also. Equation (3.4) implies that the image of $h$ is $[0, \infty)$. Hence $h$ is invertible with $h^{-1}:[0, \infty) \rightarrow[0, \infty)$. Furthermore, we easily see that

$$
\lim _{s \rightarrow \infty} \frac{h^{-1}(s)}{s}=\infty, \quad \lim _{s \rightarrow \infty} \frac{h^{-1}(s)}{s^{5}}=0
$$

by (3.4) and the fact that $\gamma^{*} \in\left(\frac{6}{5}, 2\right)$. The reader is reminded that all of these conditions are satisfied for $p(s)=s^{\gamma}, \frac{6}{5}<\gamma<2$.

Lemma 3.1. The inverse enthalpy $h^{-1}$ is locally $C^{1, \alpha}$ on $[0, \infty)$, where $\alpha=\min \left(\frac{2-\gamma}{\gamma-1}, 1\right)$. Also $h^{-1}(0)=\left(h^{-1}\right)^{\prime}(0)=0$.

Proof. $h^{-1}(0)=0$ is obvious. By $(3.2), h^{-1}(s)=O\left(s^{\frac{1}{\gamma-1}}\right)$ as $s \rightarrow 0^{+}$. Hence

$$
\left(h^{-1}\right)^{\prime}(s)=\frac{1}{h^{\prime}\left(h^{-1}(s)\right)}=O\left(s^{\frac{2-\gamma}{\gamma-1}}\right)
$$

as $s \rightarrow 0^{+}$. Since $\frac{2-\gamma}{\gamma-1}>0$ as $1<\gamma<2,\left(h^{-1}\right)^{\prime}(0)=0$ and $\left(h^{-1}\right)^{\prime}$ is continuous at zero. Also $h^{-1}$ is $C^{3}$ on $(0, \infty)$. Therefore we only need to show that $h^{-1}$ is locally $C^{1, \alpha}$ near zero. To that end, we compute for $0 \leq s_{1}<s_{2}$ sufficiently small,

$$
\left|\left(h^{-1}\right)^{\prime}\left(s_{2}\right)-\left(h^{-1}\right)^{\prime}\left(s_{1}\right)\right| \leq\left(s_{2}-s_{1}\right) \int_{0}^{1}\left|\left(h^{-1}\right)^{\prime \prime}\left(s_{1}+t\left(s_{2}-s_{1}\right)\right)\right| d t .
$$

By direct calculation we have

$$
\left(h^{-1}\right)^{\prime \prime}(s)=-\frac{h^{\prime \prime}\left(h^{-1}(s)\right)}{\left[h^{\prime}\left(h^{-1}(s)\right)\right]^{3}} .
$$

As before, we may conclude that

$$
\left(h^{-1}\right)^{\prime \prime}(s)=O\left(s^{\frac{-2 \gamma+3}{\gamma-1}}\right)=O\left(s^{\frac{2-\gamma}{\gamma-1}-1}\right)
$$

as $s \rightarrow 0^{+}$. Therefore 3.7 is bounded by

$$
C\left(s_{2}-s_{1}\right) \int_{0}^{1}\left(s_{1}+t\left(s_{2}-s_{1}\right)\right)^{\frac{2-\gamma}{\gamma-1}-1} d t .
$$

If $\frac{2-\gamma}{\gamma-1} \geq 1$, 3.10) is bounded by $C\left(s_{2}-s_{1}\right)$. If $\frac{2-\gamma}{\gamma-1}<1$, 3.10 is bounded by

$$
\begin{gathered}
C\left(s_{2}-s_{1}\right)^{\frac{2-\gamma}{\gamma-1}} \int_{0}^{1}\left(\frac{s_{1}}{s_{2}-s_{1}}+t\right)^{\frac{2-\gamma}{\gamma-1}-1} d t \\
\leq C\left(s_{2}-s_{1}\right)^{\frac{2-\gamma}{\gamma-1}} \int_{0}^{1} t^{\frac{2-\gamma}{\gamma-1}-1} d t \leq C\left(s_{2}-s_{1}\right)^{\frac{2-\gamma}{\gamma-1}}
\end{gathered}
$$

because $\frac{2-\gamma}{\gamma-1}>0$.

3.2. Properties of the radial density $\rho_{0}$. If we define

$$
u_{0}=h\left(\rho_{0}\right) \text {, }
$$

(2.13) can be rewritten as

$$
\Delta u_{0}=-4 \pi h^{-1}\left(u_{0}\right) .
$$

Lemma 3.2. There is a positive solution $u_{0} \in C^{2}\left(\overline{B_{1}}\right)$ with zero boundary condition to (3.13), which is radial, and which satisfies $\frac{\partial u_{0}}{\partial r}(r)<0$ for all $0<r \leq 1$. 
Proof. Of course, (3.13) is just a second-order ODE for radial functions. So the existence of a positive solution is classical. But to be specific, by 1 and 6 , (3.13) has a positive solution with zero boundary condition on a ball if $h^{-1}$ is locally Lipschitz, $h^{-1}(0)=0$, and satisfies (3.5). By (3.2) the enthalpy also satisfies

$$
\lim _{s \rightarrow 0^{+}} \frac{h^{-1}(s)}{s}=0
$$

By [9] a positive solution must be radial and satisfy $\frac{\partial u_{0}}{\partial r}(r)<0$ for all $0<r \leq 1$.

We have now constructed the $\rho_{0}$ in Proposition 2.1. The next lemma establishes the claimed regularity of $\rho_{0}$.

Lemma 3.3. $\rho_{0} \in C^{1, \alpha}\left(\mathbb{R}^{3}\right)$ if it is extended to be zero outside $B_{1}$, where $\alpha$ is given as in Lemma 3.1.

Proof. Since $\rho_{0}=h^{-1}\left(u_{0}\right), u_{0} \in C\left(\overline{B_{1}}\right)$, and $h^{-1}$ is continuous on $[0, \infty)$, we have $\rho_{0} \in$ $C\left(\overline{B_{1}}\right)$. Since $h^{-1}(0)=0, \rho_{0}$ vanishes on the boundary of $B_{1}$. Hence $\rho_{0}$ is continuous on $\mathbb{R}^{3}$. Furthermore, we have

$$
\partial_{i} \rho_{0}(x)=\left(h^{-1}\right)^{\prime}\left(u_{0}(x)\right) \partial_{i} u_{0}(x)
$$

Using the fact that $\partial_{i} u_{0} \in C^{1}\left(\overline{B_{1}}\right)$ and $\left(h^{-1}\right)^{\prime}$ is locally $C^{\alpha}$ on $[0, \infty)$, we easily get $\partial_{i} \rho_{0} \in$ $C^{\alpha}\left(\overline{B_{1}}\right)$. Since $\left(h^{-1}\right)^{\prime}(0)=0, \partial_{i} \rho_{0}$ vanishes on the boundary of $B_{1}$. Hence $\rho_{0} \in C^{1, \alpha}\left(\mathbb{R}^{3}\right)$.

3.3. Properties of the dilating function $g_{\zeta}$. The basic intuition is that $g_{\zeta}$, defined in (2.16), should be close to the identity map when $\|\zeta\|_{X}$ is sufficiently small. More precisely,

Lemma 3.4. There is a $C>0$ such that if $\zeta \in B_{\epsilon}(X)$ and $\epsilon$ is small enough, then $g_{\zeta}$ : $\overline{B_{1}} \rightarrow g_{\zeta}\left(\overline{B_{1}}\right)$ is a homeomorphism, as well as a $C^{1}$ diffeomorphism on $\overline{B_{1}} \backslash\{0\}$. Denoting the Jacobian matrix by $D g_{\zeta}$, the following estimates hold.

$$
\begin{gathered}
\left|D g_{\zeta}(x)-I\right|<C\|\zeta\|_{X} \quad \text { for all } x \in \overline{B_{1}} \backslash\{0\} . \\
\left|\left(D g_{\zeta}\right)^{-1}(y)-I\right|<C\|\zeta\|_{X} \quad \text { for all } y \in g_{\zeta}\left(\overline{B_{1}}\right) \backslash\{0\} . \\
\left|\operatorname{det} D g_{\zeta}(x)-1\right|<C\|\zeta\|_{X} \quad \text { for all } x \in \overline{B_{1}} \backslash\{0\} . \\
\left|g_{\zeta}(x)-x\right| \leq\|\zeta\|_{X}|x| \quad \text { for all } x \in \overline{B_{1}} . \\
\left|g_{\zeta}^{-1}(y)-y\right| \leq C\|\zeta\|_{X}|y| \quad \text { for all } y \in g_{\zeta}\left(\overline{B_{1}}\right) . \\
\left|\left(g_{\zeta}(x)-g_{\zeta}\left(x^{\prime}\right)\right)-\left(x-x^{\prime}\right)\right| \leq C\|\zeta\|_{X}\left|x-x^{\prime}\right| \quad \text { for all } x, x^{\prime} \in \overline{B_{1}} .
\end{gathered}
$$

Proof. The continuity of $g_{\zeta}$ is obvious. To study the inverse, we employ a more careful estimate of $g_{\zeta}(x)-g_{\zeta}\left(x^{\prime}\right)$. Before we present the estimate, an elementary but useful geometric fact will be established. For any point $x \in \mathbb{R}^{3}$, let $O b(x)$ be the symmetry orbit of $x$, i.e., the intersection of

(1) the cylinder about the $x_{3}$-axis through $x$ and

(2) the ball centered at 0 with radius $|x|$. 
We claim that if $x, x^{\prime} \in \mathbb{R}^{3}$ be such that $|x| \geq\left|x^{\prime}\right|$, then there is an $x^{\prime \prime} \in O b\left(x^{\prime}\right)$ such that

$$
\left|x-x^{\prime \prime}\right| \leq\left|x-x^{\prime}\right|
$$

and for any $\bar{x}$ on the line segment connecting $x$ with $x^{\prime \prime}$,

$$
\left|x^{\prime}\right|=\left|x^{\prime \prime}\right| \leq \sqrt{2}|\bar{x}| \text {. }
$$

To prove the claim, (3.23) is obvious if $x^{\prime}=0$, so we assume $x^{\prime} \neq 0$. Without loss of generality, assume $x$ is in the $x_{2} x_{3}$-plane. Then $O b\left(x^{\prime}\right)$ intersects the $x_{2} x_{3}$-plane at several points (two points if $x^{\prime}$ is on the $x_{3}$-axis, and four points if $x^{\prime}$ is not on the $x_{3}$ axis). Choose $x^{\prime \prime}$ among these points such that $x^{\prime \prime}$ and $x$ belong to the same quadrant (including boundary) of the $x_{2} x_{3}$-plane. That (3.22) holds is easy to see. Note that $x^{\prime \prime}$ is on the circle in the $x_{2} x_{3}{ }^{-}$ plane with radius $\left|x^{\prime \prime}\right|$ and $x$ is outside this circle but in the same quadrant with $x^{\prime \prime}$. It is easy to see that for any such pairs of $x$ and $x^{\prime \prime}$ and any $\bar{x}$ between the two, the smallest $|\bar{x}|$ is given by $\frac{1}{\sqrt{2}}\left|x^{\prime \prime}\right|$. This proves the claim.

Noticing the elementary inequality

$$
\frac{|\zeta(x)|}{|x|^{2}} \leq C\|\zeta\|_{X}
$$

from (2.21), we now estimate

$$
g_{\zeta}(x)-g_{\zeta}\left(x^{\prime}\right)=\left(x-x^{\prime}\right)\left(1+\frac{\zeta(x)}{|x|^{2}}\right)+x^{\prime}\left(\frac{\zeta(x)}{|x|^{2}}-\frac{\zeta\left(x^{\prime}\right)}{\left|x^{\prime}\right|^{2}}\right)
$$

as

$$
\left|g_{\zeta}(x)-g_{\zeta}\left(x^{\prime}\right)-\left(x-x^{\prime}\right)\right| \leq C\left(1+\|\zeta\|_{X}\right)\left|x-x^{\prime}\right|+\left|x^{\prime}\right|\left|\frac{\zeta(x)}{|x|^{2}}-\frac{\zeta\left(x^{\prime}\right)}{\left|x^{\prime}\right|^{2}}\right|
$$

To estimate $\left|x^{\prime}\right|\left|\frac{\zeta(x)}{|x|^{2}}-\frac{\zeta\left(x^{\prime}\right)}{\left|x^{\prime}\right|^{2}}\right|$, we assume without loss of generality that $|x| \geq\left|x^{\prime}\right|$, and use the claim to find an $x^{\prime \prime} \in O b\left(x^{\prime}\right)$ such that (3.22) and (3.23) hold. Now

$$
\begin{aligned}
& \left|x^{\prime}\right|\left|\frac{\zeta(x)}{|x|^{2}}-\frac{\zeta\left(x^{\prime}\right)}{\left|x^{\prime}\right|^{2}}\right|=\left|x^{\prime}\right|\left|\frac{\zeta(x)}{|x|^{2}}-\frac{\zeta\left(x^{\prime \prime}\right)}{\left|x^{\prime \prime}\right|^{2}}\right| \\
\leq & \left|x^{\prime}\right|\left|\frac{\nabla \zeta(\bar{x})}{\left|\bar{x}^{2}\right|}-2 \zeta(\bar{x}) \frac{\bar{x}}{|\bar{x}|^{4}}\right|\left|x-x^{\prime \prime}\right| \leq C\left|x^{\prime}\right| \frac{\|\zeta\|_{X}}{|\bar{x}|}\left|x-x^{\prime}\right| \leq C\|\zeta\|_{X}\left|x-x^{\prime}\right| .
\end{aligned}
$$

By (3.26) we now have (3.21). Estimate (3.21) in particular shows that $\left|g_{\zeta}(x)-g_{\zeta}\left(x^{\prime}\right)\right| \geq$ $\frac{1}{2}\left|x-x^{\prime}\right|$ if $\|\zeta\|_{X}<\epsilon$ is small enough. Thus $g_{\zeta}$ is invertible and $g_{\zeta}^{-1}$ is continuous, which implies that $g_{\zeta}$ is a homeomorphism. To see that it is a diffeomorphism away from the origin, we compute

$$
D g_{\zeta}(x)=\left(1+\frac{\zeta(x)}{|x|^{2}}\right) I+x \otimes\left(\frac{\nabla \zeta(x)}{|x|^{2}}-2 \zeta(x) \frac{x}{|x|^{4}}\right) .
$$

(3.16) is now obvious, which implies that $D g_{\zeta}(x)$ is invertible when $x$ is away from 0 and $\|\zeta\|_{X}<\epsilon$ is sufficiently small. Now for $y=g_{\zeta}(x)$,

$$
D g_{\zeta}^{-1}(y)=\left(D g_{\zeta}(x)\right)^{-1}=\left(I+\left(D g_{\zeta}(x)-I\right)\right)^{-1}=\sum_{k \geq 0}\left(-D g_{\zeta}(x)+I\right)^{k} .
$$

Hence (3.17) follows. (3.18) follows from (3.16) by elementary algebra. (3.19) and (3.20) can be obtained from (3.21) easily by setting $x^{\prime}=0$.

It is crucial in our method to extend $\zeta$ to the entire $\mathbb{R}^{3}$ in a way that the symmetry and good properties of $g_{\zeta}$ are preserved. Such an extension is summarized in the following lemma.

Lemma 3.5. Let $\tilde{X}$ be the same space as $X$ except that the $B_{1}$ 's in the definition of $X$ are replaced by $\mathbb{R}^{3}$. There is a bounded linear map $T$ from $X$ to $\tilde{X}$ such that for all $\zeta \in X$ :

(1) $T \zeta$ is supported in $B_{2}$. 
(2) $T \zeta(x)=\zeta(x)$ for $x \in \overline{B_{1}}$.

Proof. First of all, an extension $\zeta_{1}$ of $\zeta$ satisfying everything in the statement of this lemma except the symmetry requirement exists with suitable bounds on the derivatives. This can be accomplished by a partition of unity and the so-called higher-order reflection (see [7], for instance). Once $\zeta_{1}$ is constructed, a symmetrized version of it can be obtained by

$$
\zeta_{2}(x)=\frac{1}{4 \pi} \int_{0}^{2 \pi}\left[\zeta_{1}\left(T_{\theta} x\right)+\zeta_{1}\left(T_{\theta} T x\right)\right] d \theta,
$$

where

$$
T_{\theta}\left(x_{1}, x_{2}, x_{3}\right)=\left(x_{1} \cos \theta+x_{2} \sin \theta,-x_{1} \sin \theta+x_{2} \cos \theta, x_{3}\right),
$$

and

$$
T\left(x_{1}, x_{2}, x_{3}\right)=\left(x_{1}, x_{2},-x_{3}\right) .
$$

We easily verify that $\zeta_{2}$ is a $C^{1}$ extension of $\zeta$ satisfying all the conditions required by the lemma, for which the map $\zeta \mapsto \zeta_{2}$ is linear.

By a slight abuse of notation, we will still write $\zeta$ in place of the extended function $T \zeta$. Thus in the following presentation, $\zeta$ is considered to be defined on the entire $\mathbb{R}^{3} . g_{\zeta}$ will be a homeomorphism on $\mathbb{R}^{3}$ and a $C^{1}$ diffeomorphism on $\mathbb{R}^{3} \backslash\{0\}$, where all the estimates in Lemma 3.4 are satisfied for all $x \in \mathbb{R}^{3}$.

Notice that $g_{\zeta}(x)-x$ is linear in $\zeta$. Therefore all of the estimates in Lemma 3.4 can be applied to differences of $g_{\zeta_{1}}$ and $g_{\zeta_{2}}$, provided that $\zeta_{1}, \zeta_{2} \in B_{\epsilon}(X)$. In particular,

$$
\begin{gathered}
\left|D g_{\zeta_{1}}(x)-D g_{\zeta_{2}}(x)\right|<C\left\|\zeta_{1}-\zeta_{2}\right\|_{X} \quad \text { for all } x \in \mathbb{R}^{3} \backslash\{0\} . \\
\left|D g_{\zeta_{1}}^{-1}(y)-D g_{\zeta_{2}}^{-1}(y)\right|<C\left\|\zeta_{1}-\zeta_{2}\right\|_{X} \quad \text { for all } y \in \mathbb{R}^{3} \backslash\{0\} .
\end{gathered}
$$

$$
\begin{gathered}
\left|\operatorname{det} D g_{\zeta_{1}}(x)-\operatorname{det} D g_{\zeta_{2}}(x)\right|<C\left\|\zeta_{1}-\zeta_{2}\right\|_{X} \quad \text { for all } x \in \mathbb{R}^{3} \backslash\{0\} \\
\left|g_{\zeta_{1}}(x)-g_{\zeta_{2}}(x)\right| \leq\left\|\zeta_{1}-\zeta_{2}\right\|_{X}|x| \quad \text { for all } x \in \mathbb{R}^{3} . \\
\left|g_{\zeta_{1}}^{-1}(y)-g_{\zeta_{2}}^{-1}(y)\right| \leq C\left\|\zeta_{1}-\zeta_{2}\right\|_{X}|y| \quad \text { for all } y \in \mathbb{R}^{3} .
\end{gathered}
$$

To estimate the $X$ norm of the Fréchet derivative, the following lemma will be useful.

Lemma 3.6. Let $f$ be a continuous function on $B_{2}$ which is axisymmetric about the $x_{3}$-axis and is even in $x_{3}$. Furthermore assume

$$
|f(y)-f(0)| \leq C_{f}|y|
$$

for $y \in B_{2}$. Let

$$
V_{f}(y)=\int_{B_{2}} f\left(y^{\prime}\right) \frac{-\left(y-y^{\prime}\right)}{\left|y-y^{\prime}\right|^{3}} d y^{\prime} .
$$

Then for some constant $C>0$,

$$
\left|V_{f}\left(g_{\zeta}(x)\right)\right| \leq C\left(C_{f}+|f(0)|\right)\left(1+\|\zeta\|_{X}\right)|x|,
$$

and for any $0<\beta<1$, there exists a constant $C_{\beta}$ such that

$$
\left|V_{f}\left(g_{\zeta_{1}}(x)\right)-V_{f}\left(g_{\zeta_{2}}(x)\right)\right| \leq C_{\beta}\left(C_{f}+|f(0)|\right)\left\|\zeta_{1}-\zeta_{2}\right\|_{X}^{\beta}|x|
$$

for $x \in \overline{B_{1}}$ if $\zeta, \zeta_{1}, \zeta_{2} \in B_{\epsilon}(X)$ and $\epsilon$ is small enough. 
Proof. The proof of this lemma is similar to that of Lemma 3.4 in [17. We have

$$
V_{f}\left(g_{\zeta}(x)\right)=f(0) \int_{B_{2}} \frac{-\left(g_{\zeta}(x)-y^{\prime}\right)}{\left|g_{\zeta}(x)-y^{\prime}\right|^{3}} d y^{\prime}+\int_{B_{2}}\left(f\left(y^{\prime}\right)-f(0)\right) \frac{-\left(g_{\zeta}(x)-y^{\prime}\right)}{\left|g_{\zeta}(x)-y^{\prime}\right|^{3}} d y^{\prime}
$$

For the first term we estimate

$$
\begin{aligned}
\left|\int_{B_{2}} \frac{-\left(g_{\zeta}(x)-y^{\prime}\right)}{\left|g_{\zeta}(x)-y^{\prime}\right|^{3}} d y^{\prime}\right| & =\left|\int_{\partial B_{2}} \frac{1}{\left|g_{\zeta}(x)-y^{\prime}\right|} n\left(y^{\prime}\right) d \sigma\left(y^{\prime}\right)\right| \\
=\left|\int_{\partial B_{2}}\left(\frac{1}{\left|g_{\zeta}(x)-y^{\prime}\right|}-\frac{1}{\left|y^{\prime}\right|}\right) n\left(y^{\prime}\right) d \sigma\left(y^{\prime}\right)\right| & \leq C \int_{\partial B_{2}} \frac{1}{\left|\theta g_{\zeta}(x)-y^{\prime}\right|^{2}}\left|g_{\zeta}(x)\right| d \sigma\left(y^{\prime}\right) \\
\leq C \frac{1}{\left(2-\left(1+C\|\zeta\|_{x}\right)\right)^{2}}\left(1+\|\zeta\|_{X}\right)|x| & \leq C\left(1+\|\zeta\|_{X}\right)|x|,
\end{aligned}
$$

where $\theta \in(0,1)$ and the penultimate step follows by noticing that $\left|y^{\prime}\right|=2$ and $\left|g_{\zeta}(x)\right| \leq$ $1+C\|\zeta\|_{X}$ for $|x| \leq 1$. On the other hand, by the symmetry of $f$ and $\zeta$, we have

$$
\int_{B_{2}}\left(f\left(y^{\prime}\right)-f(0)\right) \frac{y^{\prime}}{\left|y^{\prime}\right|^{3}} d y^{\prime}=0 .
$$

Hence

$$
\begin{aligned}
& \left|\int_{B_{2}}\left(f\left(y^{\prime}\right)-f(0)\right) \frac{-\left(g_{\zeta}(x)-y^{\prime}\right)}{\left|g_{\zeta}(x)-y^{\prime}\right|^{3}} d y^{\prime}\right|=\left|\int_{B_{2}}\left(f\left(y^{\prime}\right)-f(0)\right)\left(\frac{-\left(g_{\zeta}(x)-y^{\prime}\right)}{\left|g_{\zeta}(x)-y^{\prime}\right|^{3}}-\frac{y^{\prime}}{\left|y^{\prime}\right|^{3}}\right) d y^{\prime}\right| \\
\leq & C_{f}\left(\left|\int_{\left|y^{\prime}\right| \leq 2|x|}\right| y^{\prime}\left|\left(\frac{-\left(g_{\zeta}(x)-y^{\prime}\right)}{\left|g_{\zeta}(x)-y^{\prime}\right|^{3}}-\frac{y^{\prime}}{\left|y^{\prime}\right|^{3}}\right) d y^{\prime}\right|+\left|\int_{2|x| \leq\left|y^{\prime}\right| \leq 2}\right| y^{\prime}\left|\left(\frac{-\left(g_{\zeta}(x)-y^{\prime}\right)}{\left|g_{\zeta}(x)-y^{\prime}\right|^{3}}-\frac{y^{\prime}}{\left|y^{\prime}\right|^{3}}\right) d y^{\prime}\right|\right) \\
\leq & C_{f}\left(I_{1}+I_{2}\right) .
\end{aligned}
$$

Since $\left|g_{\zeta}(x)\right| \leq\left(1+\|\zeta\|_{X}\right)|x|$ by (3.19), the ball $\left\{\left|y^{\prime}\right| \leq 2|x|\right\}$ is contained in the ball $\left\{\mid y^{\prime}-\right.$ $\left.g_{\zeta}(x)|\leq 4| x \mid\right\}$. Thus

$$
I_{1} \leq 2 \int_{\left|y^{\prime}\right| \leq 4|x|} \frac{1}{\left|y^{\prime}\right|^{2}} d y^{\prime} \leq C|x| .
$$

We observe that $\left|y^{\prime}\right|-\left|g_{\zeta}(x)\right|$ is comparable to $\left|y^{\prime}\right|$ when $\left|y^{\prime}\right| \geq 2\left|x^{\prime}\right|$ and $\|\zeta\|_{X}$ is small. By the mean value theorem,

$$
I_{2} \leq \int_{2|x| \leq\left|y^{\prime}\right| \leq 2} \frac{\left|y^{\prime}\right|\left(1+\|\zeta\|_{X}\right)|x|}{\left|\theta g_{\zeta}(x)-y^{\prime}\right|^{3}} d y^{\prime} \leq C\left(1+\|\zeta\|_{X}\right)|x| \int_{\left|y^{\prime}\right| \leq 2} \frac{1}{\left|y^{\prime}\right|^{2}} d y^{\prime}
$$

Thus (3.41) follows. Similarly

$$
\begin{aligned}
& V_{f}\left(g_{\zeta_{1}}(x)\right)-V_{f}\left(g_{\zeta_{2}}(x)\right) \\
= & f(0) \int_{B_{2}}\left(\frac{-\left(g_{\zeta_{1}}(x)-y^{\prime}\right)}{\left|g_{\zeta_{1}}(x)-y^{\prime}\right|^{3}}-\frac{-\left(g_{\zeta_{2}}(x)-y^{\prime}\right)}{\left|g_{\zeta_{2}}(x)-y^{\prime}\right|^{3}}\right) d y^{\prime} \\
& +\int_{B_{2}}\left(f\left(y^{\prime}\right)-f(0)\right)\left(\frac{-\left(g_{\zeta_{1}}(x)-y^{\prime}\right)}{\left|g_{\zeta_{1}}(x)-y^{\prime}\right|^{3}}-\frac{-\left(g_{\zeta_{2}}(x)-y^{\prime}\right)}{\left|g_{\zeta_{2}}(x)-y^{\prime}\right|^{3}}\right) d y^{\prime},
\end{aligned}
$$

We can estimate the first term as before:

$$
\begin{aligned}
& \left|\int_{B_{2}}\left(\frac{-\left(g_{\zeta_{1}}(x)-y^{\prime}\right)}{\left|g_{\zeta_{1}}(x)-y^{\prime}\right|^{3}}-\frac{-\left(g_{\zeta_{2}}(x)-y^{\prime}\right)}{\left|g_{\zeta_{2}}(x)-y^{\prime}\right|^{3}}\right) d y^{\prime}\right| \leq \int_{\partial B_{2}}\left|\frac{1}{\left|g_{\zeta_{1}}(x)-y^{\prime}\right|}-\frac{1}{\left|g_{\zeta_{2}}(x)-y^{\prime}\right|}\right| d \sigma\left(y^{\prime}\right) \\
\leq & C \int_{\partial B_{2}} \frac{1}{\left|\theta g_{\zeta_{1}}(x)+(1-\theta) g_{\zeta_{2}}(x)-y^{\prime}\right|^{2}}\left|g_{\zeta_{1}}(x)-g_{\zeta_{2}}(x)\right| d \sigma\left(y^{\prime}\right) \leq C\left\|\zeta_{1}-\zeta_{2}\right\|_{X}|x|,
\end{aligned}
$$

where we have used (3.36) to get the last step. The second term of (3.47) can be estimated as follows. Let $\bar{x}=\frac{1}{2}\left(g_{\zeta_{1}}(x)+g_{\zeta_{2}}(x)\right)$, and $\delta=\left\|\zeta_{1}-\zeta_{2}\right\|_{X}$. By (3.36),$\delta|x|$ is no less than 
$2\left|g_{\zeta_{1}}(x)-\bar{x}\right|$ or $2\left|g_{\zeta_{2}}(x)-\bar{x}\right|$.

$$
\begin{aligned}
& \left|\int_{B_{2}}\left(f\left(y^{\prime}\right)-f(0)\right)\left(\frac{-\left(g_{\zeta_{1}}(x)-y^{\prime}\right)}{\left|g_{\zeta_{1}}(x)-y^{\prime}\right|^{3}}-\frac{-\left(g_{\zeta_{2}}(x)-y^{\prime}\right)}{\left|g_{\zeta_{2}}(x)-y^{\prime}\right|^{3}}\right) d y^{\prime}\right| \\
\leq & C_{f}\left|\int_{B_{2}}\right| y^{\prime}\left|\left(\frac{-\left(g_{\zeta_{1}}(x)-y^{\prime}\right)}{\left|g_{\zeta_{1}}(x)-y^{\prime}\right|^{3}}-\frac{-\left(g_{\zeta_{2}}(x)-y^{\prime}\right)}{\left|g_{\zeta_{2}}(x)-y^{\prime}\right|^{3}}\right) d y^{\prime}\right| .
\end{aligned}
$$

Assuming $\left\|\zeta_{1}\right\|_{X}$ and $\left\|\zeta_{2}\right\|_{X}$ are small, we now split the integral into three pieces $I_{1}+I_{2}+I_{3}$, on the regions $\left\{\left|y^{\prime}-\bar{x}\right| \leq \delta|x|\right\},\left\{\delta|x| \leq\left|y^{\prime}-\bar{x}\right| \leq 2|x|\right\}$, and $\left\{\left|y^{\prime}-\bar{x}\right| \geq 2|x|\right\}$, all within the ball $\left\{\left|y^{\prime}\right| \leq 2\right\}$. Since the ball $\left\{\left|y^{\prime}-\bar{x}\right| \leq \delta|x|\right\}$ is contained in the balls $\left\{\left|y^{\prime}-g_{\zeta_{1}}(x)\right| \leq 4 \delta|x|\right\}$ and $\left\{\left|y^{\prime}-g_{\zeta_{2}}(x)\right| \leq 4 \delta|x|\right\}$, we have

$$
I_{1} \leq C \int_{\left|y^{\prime}\right| \leq 4 \delta} \frac{1}{\left|y^{\prime}\right|^{2}} d y^{\prime} \leq C \delta|x|=C\left\|\zeta_{1}-\zeta_{2}\right\|_{X}|x| .
$$

On the other hand, when $\left|y^{\prime}-\bar{x}\right| \geq \delta|x|,\left|y^{\prime}-\bar{x}\right|$ is comparable to $\left|y^{\prime}-\theta g_{\zeta_{1}}(x)-(1-\theta) g_{\zeta_{2}}(x)\right|$ for every $\theta \in(0,1)$. By the mean value theorem,

$$
\begin{aligned}
I_{2} & \leq C \int_{\delta|x| \leq\left|y^{\prime}-\bar{x}\right| \leq 2|x|} \frac{\left|g_{\zeta_{1}}(x)-g_{\zeta_{2}}(x)\right|}{\left|\theta g_{\zeta_{1}}(x)+(1-\theta) g_{\zeta_{2}}(x)-y^{\prime}\right|^{3}} d y^{\prime} \\
& \leq C \int_{\delta|x| \leq\left|y^{\prime}-\bar{x}\right| \leq 2|x|} \frac{\left|g_{\zeta_{1}}(x)-g_{\zeta_{2}}(x)\right|}{\left|y^{\prime}-\bar{x}\right|^{3}} d y^{\prime} \\
& \leq C\left(\log \frac{2}{\delta}\right) \delta|x| \\
& \leq C_{\beta}\left\|\zeta_{1}-\zeta_{2}\right\|_{X}^{\beta}|x|
\end{aligned}
$$

for every $\beta \in(0,1)$. Finally, when $\left|y^{\prime}-\bar{x}\right| \geq 2|x|,\left|y^{\prime}\right|$ is comparable to $\mid y^{\prime}-\theta g_{\zeta_{1}}(x)-(1-$ $\theta) g_{\zeta_{2}}(x) \mid$ for every $\theta \in(0,1)$. Again by the mean value theorem,

$$
\begin{aligned}
I_{3} & \leq C \int_{\left|y^{\prime}-\bar{x}\right| \geq 2|x|,\left|y^{\prime}\right| \leq 2} \frac{\left|y^{\prime}\right|\left|g_{\zeta_{1}}(x)-g_{\zeta_{2}}(x)\right|}{\left|\theta g_{\zeta_{1}}(x)+(1-\theta) g_{\zeta_{2}}(x)-y^{\prime}\right|^{3}} d y^{\prime} \\
& \leq C \int_{\left|y^{\prime}\right| \leq 2} \frac{\left|g_{\zeta_{1}}(x)-g_{\zeta_{2}}(x)\right|}{\left|y^{\prime}\right|^{2}} d y^{\prime} \\
& \leq C\left\|\zeta_{1}-\zeta_{2}\right\| X|x|
\end{aligned}
$$

Thus (3.42) follows.

\section{EULER MODEL: ANALYSIS OF THE LINEARIZED OPERATOR}

In this section we prove the main theorems (Theorem 2.1 and Theorem 2.2.).

4.1. Linearization. We postpone calculating the Fréchet derivative of $\mathcal{F}(\zeta, \kappa)$ until the next section. $\mathcal{F}$ has three terms shown in (5.1) given in (5.2)-(5.4). The formula for $\frac{\partial \mathcal{F}}{\partial \zeta}$ is given in (5.17)-(5.21). We freely use that result here.

It is the operator $\mathcal{L}=\frac{\partial \mathcal{F}}{\partial \zeta}(0,0)$ that requires detailed analysis. We write $\mathcal{F}$ as $\mathcal{M F}_{1}+$ $\kappa \mathcal{F}_{2}+\mathcal{F}_{3}$ as in (5.1), where $\mathcal{M}, \mathcal{F}_{1}, \mathcal{F}_{2}, \mathcal{F}_{3}$ are given in (5.2)-(5.4). First, by Lemma 5.2 and (5.18) we have

$$
\mathcal{M}^{\prime}(0) \xi=-\frac{1}{M} \int_{B_{1}} \rho_{0}(x) \nabla \cdot\left(\xi(x) \frac{x}{|x|^{2}}\right) d x=\frac{1}{M} \int_{B_{1}} \frac{\rho_{0}^{\prime}(x)}{|x|} \xi(x) d x .
$$

Here we integrated by parts once, and $\rho_{0}^{\prime}$ denotes the radial derivative of $\rho_{0}$. By (5.2),

$$
\mathcal{F}_{1}(0)(x)=\int_{B_{1}} \rho_{0}(y)\left(\frac{1}{|x-y|}-\frac{1}{|y|}\right) d y=h\left(\rho_{0}\right)(x)-h\left(\rho_{0}\right)(0) .
$$


The last equality is a consequence of the fact that $\mathcal{F}(0,0)=0$, which is implied by

$$
\int_{B_{1}} \rho_{0}(y) \frac{1}{|x-y|} d y-h\left(\rho_{0}\right)(x)=\text { const },
$$

which is a restatement of (2.13). By (5.19),

$$
\left[\mathcal{F}_{1}^{\prime}(0) \xi\right](x)=\int_{B_{1}}-\frac{\rho_{0}^{\prime}(y)}{|y|} \xi(y)\left(\frac{1}{|x-y|}-\frac{1}{|y|}\right) d y+\int_{B_{1}} \rho_{0}(y) \frac{-(x-y)}{|x-y|^{3}} d y \cdot \xi(x) \frac{x}{|x|^{2}}
$$

The last term in (4.4) can be simplified as

$$
\int_{B_{1}} \rho_{0}(y) \frac{-(x-y)}{|x-y|^{3}} d y \cdot \frac{x}{|x|^{2}}=\frac{x}{|x|^{2}} \cdot \nabla_{x} \int_{B_{1}} \rho_{0}(y) \frac{1}{|x-y|} d y=\frac{1}{|x|}\left(h\left(\rho_{0}\right)^{\prime}(x)\right)=\frac{1}{|x|} u_{0}^{\prime}(x) .
$$

Here prime (') means radial derivative. (4.4) now becomes

$$
\left[\mathcal{F}_{1}^{\prime}(0) \xi\right](x)=\int_{B_{1}}-\frac{\rho_{0}^{\prime}(y)}{|y|} \xi(y)\left(\frac{1}{|x-y|}-\frac{1}{|y|}\right) d y+\frac{u_{0}^{\prime}(x)}{|x|} \xi(x) .
$$

By (5.21),

$$
\left[\mathcal{F}_{3}^{\prime}(0) \xi\right](x)=\left(h^{\prime}\left(\rho_{0}\right) \rho_{0}(0)-h^{\prime}\left(\rho_{0}\right) \rho_{0}(x)\right) \mathcal{M}^{\prime}(0) \xi .
$$

Combining the previous equations as in Lemma [5.2, we have the following formula for the linearized operator around zero.

$$
\begin{aligned}
& {[\mathcal{L} \xi](x)=\left[\frac{\partial F}{\partial \zeta}(0,0) \xi\right](x)} \\
& =\frac{u_{0}^{\prime}(x)}{|x|} \xi(x)-\int_{B_{1}} \frac{\rho_{0}^{\prime}(y)}{|y|} \xi(y)\left(\frac{1}{|x-y|}-\frac{1}{|y|}\right) d y+\frac{k\left(\rho_{0}\right)(x)-k\left(\rho_{0}\right)(0)}{M} \int_{B_{1}} \frac{\rho_{0}^{\prime}(y)}{|y|} \xi(y) d y,
\end{aligned}
$$

where $k(s)=h(s)-s h^{\prime}(s)$.

4.2. Radial part of the Euler kernel. In the next two subsections we prove the following theorem in terms of the mass function defined in (4.24).

Theorem 4.1. The linearized operator $\mathcal{L}$ is injective if and only if $M^{\prime}\left(u_{0}(0)\right) \neq 0$.

We begin by proving that there is no nonzero radial function in the nullspace $N(\mathcal{L})$ if and only if $M^{\prime}\left(u_{0}(0)\right) \neq 0$. This is the most delicate part of the analysis. It is significantly more subtle than the corresponding analysis in [10].

From (4.8) we have, for any $\xi \in N(\mathcal{L})$,

$$
\begin{gathered}
0=\frac{u_{0}^{\prime}(x)}{|x|} \xi(x)-\int_{B_{1}} \frac{\rho_{0}^{\prime}(y)}{|y|} \xi(y)\left(\frac{1}{|x-y|}-\frac{1}{|y|}\right) d y \\
+\frac{1}{M}\left[k\left(\rho_{0}\right)(x)-k\left(\rho_{0}\right)(0)\right] \int_{B_{1}} \frac{\rho_{0}^{\prime}(y)}{|y|} \xi(y) d y .
\end{gathered}
$$

We now assume that $\xi \in X$ is radial. Let $\alpha=\frac{u_{0}^{\prime}}{|x|} \xi$. Then $\alpha$ is also radial. Applying $\Delta$ to both sides of (4.9), we have

$$
\Delta \alpha+4 \pi \frac{\rho_{0}^{\prime}}{u_{0}^{\prime}} \alpha+\frac{1}{M} \Delta\left(k\left(\rho_{0}\right)\right) \int_{B_{1}} \frac{\rho_{0}^{\prime}(y)}{u_{0}^{\prime}(y)} \alpha(y) d y=0 .
$$

Integrating this equation on $B_{1}$ and using prime $\left(^{\prime}\right)$ to denote the radial derivative, we get

$$
\alpha^{\prime}(1)=-\left(1+\frac{1}{M}\left(k\left(\rho_{0}\right)\right)^{\prime}(1)\right) \int_{B_{1}} \frac{\rho_{0}^{\prime}(y)}{u_{0}^{\prime}(y)} \alpha(y) d y .
$$


On the other hand, since $k\left(\rho_{0}\right)=h\left(\rho_{0}\right)-\rho_{0} h^{\prime}\left(\rho_{0}\right)=u_{0}-\frac{u_{0}^{\prime}}{\rho_{0}^{\prime}} \rho_{0}$ and $\Delta u_{0}=-4 \pi \rho_{0}$, we have

$$
\Delta\left(u_{0}-k\left(\rho_{0}\right)\right)+4 \pi \frac{\rho_{0}^{\prime}}{u_{0}^{\prime}}\left(u_{0}-k\left(\rho_{0}\right)\right)+\Delta\left(k\left(\rho_{0}\right)\right)=0 .
$$

Integrating (3.13) over $B_{1}$, we have $u_{0}^{\prime}(1)=-\int_{B_{1}} \rho_{0} d x=-M$, so that

$$
\left(u_{0}-k\left(\rho_{0}\right)\right)^{\prime}(1)=u_{0}^{\prime}(1)-\left(k\left(\rho_{0}\right)\right)^{\prime}(1)=-M-\left(k\left(\rho_{0}\right)\right)^{\prime}(1) .
$$

Therefore if we let $\beta=\left(u_{0}-k\left(\rho_{0}\right)\right) \frac{1}{M} \int_{B_{1}} \frac{\rho_{0}^{\prime}(y)}{u_{0}^{\prime}(y)} \alpha(y) d y$, then (4.12) becomes

$$
\Delta \beta+4 \pi \frac{\rho_{0}^{\prime}}{u_{0}^{\prime}} \beta+\frac{1}{M} \Delta\left(k\left(\rho_{0}\right)\right) \int_{B_{1}} \frac{\rho_{0}^{\prime}(y)}{u_{0}^{\prime}(y)} \alpha(y) d y=0 .
$$

This is the same equation as satisfied by $\alpha$. Furthermore from (4.11) and (4.13), $\beta^{\prime}(1)=$ $\alpha^{\prime}(1)$. Therefore the difference $w=\alpha-\beta$ satisfies

$$
\Delta w+4 \pi \frac{\rho_{0}^{\prime}}{u_{0}^{\prime}} w=0, \quad w^{\prime}(1)=0 .
$$

Lemma 4.1. The following statements are equivalent:

(1) There is no nonzero radial solution to 4.15).

(2) There is no nonzero radial function in $N(\mathcal{L})$.

Proof. Suppose $w=0$ is the only radial solution to (4.15), then $\alpha=\beta$. But $\alpha(0)=0$, so that, by definition of $\beta$, we would have

$$
0=\beta(0) \frac{M}{\int_{B_{1}} \rho_{0}^{\prime} \alpha / u_{0}^{\prime} d y}=\left(u_{0}-k\left(\rho_{0}\right)\right)(0)=\lim _{r \rightarrow 0} \frac{u_{0}^{\prime}(r)}{\rho_{0}^{\prime}(r)} \rho_{0}(0)=\rho_{0}(0) h^{\prime}\left(\rho_{0}(0)\right)>0 .
$$

The only possible conclusion would be that $\int_{B_{1}} \rho_{0}^{\prime} \alpha / u_{0}^{\prime} d y=0$. Looking at the definition of $\beta$, we see that $\beta$ vanishes. Hence $\alpha=0$, meaning that the only radial function in $N(\mathcal{L})$ is zero.

On the other hand, if $w$ is a nonzero solution to (4.15), we define

$$
\alpha=w+C\left(u_{0}-k\left(\rho_{0}\right)\right)=w+C \frac{u_{0}^{\prime}}{\rho_{0}^{\prime}} \rho_{0} .
$$

It follows that $C=\frac{1}{M} \int_{B_{1}} \frac{\rho_{0}^{\prime}}{u_{0}^{\prime}} \alpha d y$. In fact, integrate (4.15) on $B_{1}$ to get

$$
\int_{B_{1}} \frac{\rho_{0}^{\prime}(y)}{u_{0}^{\prime}(y)} w(y) d y=0 .
$$

Now integrate (4.16) against $\frac{\rho_{0}^{\prime}}{u_{0}^{\prime}}$ on $B_{1}$ to get

$$
\int_{B_{1}} \frac{\rho_{0}^{\prime}}{u_{0}^{\prime}} \alpha d y=C \int_{B_{1}} \rho_{0}(y) d y=C M .
$$

By (4.12) and (4.15), such an $\alpha$ satisfies (4.10). We now choose $C$ such that $\alpha(0)=0$ :

$$
0=w(0)+C \frac{u_{0}^{\prime}}{\rho_{0}^{\prime}}(0) \rho_{0}(0)
$$

and define $\xi=\frac{|x|}{u_{0}^{\prime}} \alpha$. It follows that the right hand side of (4.9) is a radial harmonic function on $B_{1}$ which vanishes at the origin, hence is identically zero. It is easy to verify that $\xi=\frac{|x|}{u_{0}^{\prime}} \alpha \in X$, hence is in $N(\mathcal{L})$.

Our goal is therefore to study uniqueness of radial solutions to (4.15). Since $w$ is radial, and $\rho_{0}=h^{-1}\left(u_{0}\right)$, we may rewrite 4.15) as

$$
w^{\prime \prime}+\frac{2}{r} w+4 \pi\left(h^{-1}\right)^{\prime}\left(u_{0}\right) w=0, \quad w^{\prime}(0)=w^{\prime}(1)=0 .
$$


Let us consider the closely related initial value problem $(v=v(r ; a))$

$$
v^{\prime \prime}+\frac{2}{r} v+4 \pi h^{-1}(v)=0, \quad v(0 ; a)=a, \quad v^{\prime}(0 ; a)=0 .
$$

Of course, $v$ is differentiable with respect to $a$. Since $u_{0}$ solves (4.21) with $a=u_{0}(0)$, we have $v\left(\cdot ; u_{0}(0)\right)=u_{0}$. Also

$$
v\left(1, u_{0}(0)\right)=u_{0}(1)=0, \quad v^{\prime}\left(1, u_{0}(0)\right)=u_{0}^{\prime}(1) \neq 0 .
$$

By the implicit function theorem, there exists a function $R=R(a)$ for which $R\left(u_{0}(0)\right)=1$ that solves $v(R, a)=0$ for $(R, a)$ in a neighborhood of $\left(1, u_{0}(0)\right)$. For such values of $(R, a)$, $v(\cdot ; a)$ is a positive solution to the equation

$$
\Delta v+4 \pi h^{-1}(v)=0
$$

on $B_{R}$ with zero boundary value at $|x|=R$. We now define the physical mass of the solution $v(\cdot ; a)$ as

$$
M(a)=\int_{B_{R(a)}} h^{-1}(v(|x|, a)) d x=\int_{0}^{R(a)} 4 \pi h^{-1}(v(r ; a)) r^{2} d r .
$$

Thus $M\left(u_{0}(0)\right)=M$. It suffices to prove the following lemma.

Lemma 4.2. (4.15) has a unique radial solution that is trivial if and only if $M^{\prime}\left(u_{0}(0)\right) \neq 0$.

The condition of the lemma means that near $\rho_{0}$, the mass of a radial solution strictly changes as the density at the origin changes.

Proof. A direct computation yields

$$
\begin{aligned}
M^{\prime}(a) & =4 \pi h^{-1}(v(R(a) ; a)) R^{2}(a) R^{\prime}(a)+\int_{0}^{R(a)} 4 \pi\left(h^{-1}\right)^{\prime}(v(s ; a)) v_{a}(s ; a) s^{2} d s \\
& =\int_{0}^{R(a)} 4 \pi\left(h^{-1}\right)^{\prime}(v(s ; a)) v_{a}(s ; a) s^{2} d s
\end{aligned}
$$

because $v(R(a) ; a)=0$ and $h^{-1}(0)=0$. In particular, putting $a=u_{0}(0)$, we have $R(a)=1$ so that

$$
M^{\prime}\left(u_{0}(0)\right)=\int_{0}^{1} 4 \pi\left(h^{-1}\right)^{\prime}\left(u_{0}(s)\right) v_{a}\left(s ; u_{0}(0)\right) s^{2} d s .
$$

On the other hand, for $a=u_{0}(0)$ the derivative $v_{a}\left(\cdot ; u_{0}(0)\right)$ solves

$$
v_{a}^{\prime \prime}+\frac{2}{r} v_{a}+4 \pi\left(h^{-1}\right)^{\prime}\left(u_{0}\right) v_{a}=0, \quad v_{a}\left(0, u_{0}(0)\right)=1, \quad v_{a}^{\prime}\left(0, u_{0}(0)\right)=0 .
$$

This is the same as the equation (4.20) satisfied by $w$. Thus $w$ must be a constant multiple of $v_{a}\left(\cdot ; u_{0}(0)\right)$. Integrating the equation $\Delta v_{a}+4 \pi\left(h^{-1}\right)^{\prime}\left(u_{0}\right) v_{a}=0$ on $B_{1}$, we get

$$
v_{a}^{\prime}\left(1 ; u_{0}(0)\right)=-\int_{0}^{1} 4 \pi\left(h^{-1}\right)^{\prime}\left(u_{0}(s)\right) v_{a}\left(s ; u_{0}(0)\right) s^{2} d s=-M^{\prime}\left(u_{0}(0)\right) .
$$

If $M^{\prime}\left(u_{0}(0)\right) \neq 0$, together with the condition $w^{\prime}(1)=0$, this implies that $w$ must be the zero multiple of $v_{a}\left(\cdot ; u_{0}(0)\right)$. So $w=0$. If $M^{\prime}\left(u_{0}(0)\right)=0$, then $w=v_{a}\left(\cdot ; u_{0}(0)\right)$ is a nontrivial radial solution to (4.15).

Remark 5. The mass function in this section is parametrized by the center value of u, while the mass function in the statement of Theorem 2.1 is parametrized by the center value of $\rho$. It is easy to see that $M^{\prime}$ is nonzero in one parametrization if and only if it is nonzero in the other, since $h^{\prime}(s)>0$ for $s>0$. 
4.3. Non-radial part of the Euler kernel. In this subsection, we treat the non-radial part of $N(\mathcal{L})$, thus concluding Theorem 4.1 .

Lemma 4.3. All elements of the nullspace of $\mathcal{L}$ are radial.

Proof. Let $\mathcal{L} \xi=0, \xi \in X$. Let $Y_{l m}(\theta)$ be the standard spherical harmonics on $\mathbb{S}^{2}$ where $l=0,1, \ldots ; m=-l, \ldots, l$. For any function $\eta(x)$, we denote its $l m$-component by $\eta_{l m}(r)=$ $\left\langle\eta, Y_{l m}\right\rangle=\int_{\mathbb{S}^{2}} \eta(r \theta) Y_{l m}(\theta) d S_{\theta}$, where we write $r=|x|$ and $\theta=\frac{x}{r}$.

It suffices to prove that $\xi_{l m}=0$ for all $l \geq 1$. The equation (4.8) satisfied by $\xi$ is

$$
0=\frac{u_{0}^{\prime}(x)}{|x|} \xi(x)-\varphi(x)+\frac{k\left(\rho_{0}\right)(x)-k\left(\rho_{0}\right)(0)}{M} \int_{B_{1}} \frac{\rho_{0}^{\prime}(y)}{|y|} \xi(y) d y
$$

where

$$
\varphi(x)=\int_{B_{1}} \frac{\rho_{0}^{\prime}(y)}{|y|} \xi(y)\left(\frac{1}{|x-y|}-\frac{1}{|y|}\right) d y .
$$

Since $\rho_{0} \in C^{1, \alpha}\left(\mathbb{R}^{3}\right)$ by Lemma 3.3 , and $\rho_{0}^{\prime}=0$ on $\partial B_{1}$, we have $\varphi \in C^{2, \alpha}\left(\mathbb{R}^{3}\right)$ and

$$
\Delta \varphi(x)=-4 \pi \frac{\rho_{0}^{\prime}(x)}{|x|} \xi(x),
$$

where the right side is taken to be zero outside $B_{1}$. Note that $\varphi_{l m}$ is bounded, radial and in $C^{2, \alpha}$ on $\mathbb{R}^{3} \backslash\{0\}$. We integrate (4.31) against $Y_{l m}$ on $\mathbb{S}^{2}$. The left side becomes

$$
\begin{gathered}
\left\langle\Delta \varphi, Y_{l m}\right\rangle=\left\langle\partial_{r}^{2} \varphi+\frac{2}{r} \partial_{r} \varphi+\frac{1}{r^{2}} \Delta_{\mathbb{S}^{2}} \varphi, Y_{l m}\right\rangle \\
=\left(\partial_{r}^{2}+\frac{2}{r} \partial_{r}\right) \varphi_{l m}+\frac{1}{r^{2}}\left\langle\varphi, \Delta_{\mathbb{S}^{2}} Y_{l m}\right\rangle=\Delta \varphi_{l m}-\frac{l(l+1)}{r^{2}} \varphi_{l m} .
\end{gathered}
$$

For $r=|x|>1$ the right side vanishes. For $0<|x|<1$, the right side is $-4 \pi \frac{\rho_{0}^{\prime}}{|x|}\left\langle\xi, Y_{l m}\right\rangle$. On the other hand, if we integrate (4.29) against $Y_{l m}$ with $l \geq 1$, we get $\frac{u_{0}^{\prime}}{|x|} \xi_{l m}=\varphi_{l m}$. Hence $-4 \pi \frac{\rho_{0}^{\prime}}{|x|} \xi_{l m}=-4 \pi \frac{\rho_{0}^{\prime}}{u_{0}^{\prime}} \varphi_{l m}$. Thus $\varphi_{l m}$ satisfies the second order equation

$$
\Delta \varphi_{l m}-\frac{l(l+1)}{r^{2}} \varphi_{l m}= \begin{cases}-4 \pi \frac{\rho_{0}^{\prime}}{u_{0}^{\prime}} \varphi_{l m} & \text { if } 0<|x|<1 \\ 0 & \text { if }|x| \geq 1\end{cases}
$$

For $r>1$ it follows that $\varphi$ is a linear combination of $r^{l}$ and $r^{-(l+1)}$. Being bounded at infinity, it must be that

$$
\varphi_{l m}(r)=C_{l m} r^{-(l+1)}
$$

when $|x| \geq 1$ for some constant $C_{l m}$.

Now consider $r<1$. Recall that $u_{0}$ and $\rho_{0}$ are related by $u_{0}^{\prime \prime}+\frac{2}{r} u_{0}^{\prime}+4 \pi \rho_{0}=\Delta u_{0}+4 \pi \rho_{0}=0$. Taking the radial derivative, we get

$$
\Delta u_{0}^{\prime}-\frac{2}{r^{2}} u_{0}^{\prime}+4 \pi \rho_{0}^{\prime}=0 .
$$

In terms of another auxiliary function $\psi_{l m}(r)=\frac{\varphi_{l m}(r)}{u_{0}^{\prime}(r)}=\frac{\xi_{l m}(r)}{r}$, 4.32) can be written for $0<r=|x|<1$ as

$$
\Delta\left(u_{0}^{\prime} \psi_{l m}\right)-\frac{l(l+1)}{r^{2}} u_{0}^{\prime} \psi_{l m}=-4 \pi \rho_{0}^{\prime} \psi_{l m}
$$

Dividing (4.35) by $u_{0}^{\prime}$ and using (4.34), we see that $\psi_{l m}$ satisfies the equation

$$
\Delta \psi_{l m}+2 \frac{u_{0}^{\prime \prime}}{u_{0}^{\prime}} \psi_{l m}^{\prime}+\frac{2-l(l+1)}{r^{2}} \psi_{l m}=0
$$

for $0<|x|<1$. It suffices to prove that $\psi_{l m}=0$ for all $l \geq 1$. 
Since $\left|\frac{\xi_{l m}(r)}{r}\right| \leq C\|\xi\|_{X} r$, we know that $\psi_{l m} \in C\left(\bar{B}_{1}\right)$ and $\psi_{l m}(0)=0$. Note that the numerator in the last term of (4.36) is $2-l(l-1) \leq 0$. Thus (4.36) allows us to apply the strong maximum principle for $0<r<1$. There is no interior positive maximum of $\psi_{l m}$. Let $\Psi$ be the maximum of $\psi_{l m}$ over $\bar{B}_{1}$.

Suppose that $\Psi>0$. Then the maximum occurs at $r=1$. Furthermore, $\psi_{l m}^{\prime}(1)>0$. But from the definition of $\psi_{l m}$ and (4.33), we have

$$
0<\Psi=\psi_{l m}(1)=\frac{C_{l m}}{u_{0}^{\prime}(1)}, \quad 0<\psi_{l m}^{\prime}(1)=\frac{C_{l m}(1-l)}{u_{0}^{\prime}(1)} .
$$

We know that $u_{0}^{\prime}(1)<0$. From these two inequalities we conclude that $C_{l m}<0$ and $1-l>0$, which is a contradiction. Therefore $\Psi \leq 0$ and $\psi_{l m} \leq 0$.

There is also no interior negative minimum of $\psi_{l m}$ and by similar reasoning we deduce that $\psi_{l m} \geq 0$. Therefore $\psi_{l m}=0$. This completes the proof.

\subsection{Compactness.}

Lemma 4.4. $\mathcal{L}: X \rightarrow X$ has the form $\mathcal{L}=J+K$ where $J$ is an isomorphism, and $K$ is a compact operator.

Proof. In fact, let

$$
[J \xi](x)=\frac{u_{0}^{\prime}(x)}{|x|} \xi(x)
$$

$$
[K \xi](x)=-\int_{B_{1}} \frac{\rho_{0}^{\prime}(y)}{|y|} \xi(y)\left(\frac{1}{|x-y|}-\frac{1}{|y|}\right) d y+\frac{1}{M}\left[k\left(\rho_{0}\right)(x)-k\left(\rho_{0}\right)(0)\right] \int \frac{\rho_{0}^{\prime}(y)}{|y|} \xi(y) d y .
$$

To show $J$ is bounded, we write the spatial derivative of $J \xi$ as

$$
\partial_{i}[J \xi](x)=\left[|x| \partial_{i}\left(\frac{u_{0}^{\prime}(x)}{|x|}\right) \frac{\xi(x)}{|x|^{2}}+\frac{u_{0}^{\prime}(x)}{|x|} \frac{\partial_{i} \xi(x)}{|x|}\right]|x| .
$$

We need the terms in the square brackets to be bounded by $C\|\xi\|_{X}$. Since $\frac{\xi(x)}{|x|^{2}}$ and $\frac{\nabla \xi(x)}{|x|}$ are both bounded by $C\|\xi\|_{X}$, we only need $|x| \partial_{i}\left(\frac{u_{0}^{\prime}(x)}{|x|}\right)$ and $\frac{u_{0}^{\prime}(x)}{|x|}$ to be bounded in $B_{1}$. This is true because $u_{0}^{\prime}(0)=0$. On the other hand, $\left[J^{-1} \xi\right](x)=\frac{|x|}{u_{0}^{\prime}(x)} \xi(x)$. As above, in order for $J^{-1}$ to be bounded, we need $|x| \partial_{i}\left(\frac{|x|}{u_{0}^{\prime}(x)}\right)$ and $\frac{|x|}{u_{0}^{\prime}(x)}$ to be bounded in $B_{1}$. This is again easy to verify with the help of Lemma 3.2 . The key facts are that $u_{0}^{\prime}(0)=0, u_{0}^{\prime \prime}(0) \neq 0$, and $u_{0}^{\prime}(r)<0$ for all $0<r \leq 1$.

Now we write $K=A+B$, where $A$ and $B$ are the two terms in (4.38). The operator $B$ has rank one, hence is compact once we verify that it maps $X$ to $X$. To that end we just need $k\left(\rho_{0}\right)(x)-k\left(\rho_{0}\right)(0)$ to belong to $X$. We compute

$$
\partial_{i} k\left(\rho_{0}\right)=k^{\prime}\left(\rho_{0}\right) \partial_{i} \rho_{0}=-\rho_{0} h^{\prime \prime}\left(\rho_{0}\right) \partial_{i} \rho_{0}=-\rho_{0} \frac{h^{\prime \prime}(\rho)}{h^{\prime}\left(\rho_{0}\right)} \partial_{i} u_{0}
$$

By (3.3) and the fact that $\left|\partial_{i} u_{0}(x)\right| \leq C|x|$, we see that $\left|\partial_{i} k\left(\rho_{0}\right)\right|$ is bounded by $C|x|$ on $B_{1}$. We now show compactness of $A$. Recall that $\frac{\rho_{0}^{\prime}(y)}{|y|} \in C^{\alpha}\left(\overline{B_{1}}\right)$ and $\|\xi\|_{C^{1}\left(\overline{B_{1}}\right)} \leq C\|\xi\|_{X}$ so that

$$
\left\|\rho_{0}^{\prime}(y) \xi(y) /|y|\right\|_{C^{\alpha}\left(\overline{B_{1}}\right)} \leq C\|\xi\|_{X} .
$$

But the standard Schauder estimates assert that

$$
\|A \xi\|_{C^{2, \alpha}\left(\overline{B_{1}}\right)} \leq C\left\|\rho_{0}^{\prime}(y) \xi(y) /|y|\right\|_{C^{\alpha}\left(\overline{B_{1}}\right)}
$$

since $\rho_{0}^{\prime}$ vanishes on the boundary of $B_{1}$. Because $C^{2, \alpha}\left(\overline{B_{1}}\right)$ is compactly embedded in $C^{2}\left(\overline{B_{1}}\right)$ and because $A \xi(0)=0$ and $\|A \xi\|_{X} \leq C\|A \xi\|_{C^{2}\left(\overline{B_{1}}\right)}$, we conclude that $A: X \rightarrow X$ is compact. 
Invoking the Fredholm alternative for $J^{-1} \mathcal{L}$ and combining the last three lemmas, we deduce that $\mathcal{L}$ is an isomorphism. By the discussion in Section 2.3 , this completes the proof of Theorem 2.1. It only remains to prove the Fréchet differentiability of $\mathcal{F}$.

4.5. Realization of the mass condition. Theorem 4.1 shows that injectivity of $\mathcal{L}$ is equivalent to the mass condition $M^{\prime}\left(u_{0}(0)\right) \neq 0$. In this subsection, we prove for the two cases in Theorem 2.2 this condition is satisfied.

First, we consider the power laws.

Lemma 4.5. Suppose $p(s)=s^{\gamma}, \gamma \in\left(\frac{6}{5}, 2\right)$. Then $M^{\prime}\left(u_{0}(0)\right) \neq 0$ if and only if $\gamma \neq \frac{4}{3}$.

Proof. We begin by noticing that $p(s)=s^{\gamma}, \gamma \in\left(\frac{6}{5}, 2\right)$ satisfies (2.8), (2.9) and (2.10). As a consequence the radial density $\rho_{0}$, and the function $u_{0}$ exist as are constructed in Section 3.2. By the proof of Lemma 4.2. $M^{\prime}\left(u_{0}(0)\right)=-v_{a}^{\prime}\left(1 ; u_{0}(0)\right)$. To find out about $v_{a}^{\prime}\left(1 ; u_{0}(0)\right)$, we compute $h^{-1}(s)=\left(\frac{\gamma-1}{\gamma} s\right)^{\frac{1}{\gamma-1}}$. By (4.21), $v(r ; a)$ solves

$$
v^{\prime \prime}+\frac{2}{r} v^{\prime}+4 \pi\left(\frac{\gamma-1}{\gamma} v\right)^{\frac{1}{\gamma-1}}=0, \quad v(0)=a, v^{\prime}(0)=0 .
$$

By a simple scaling, we see that

$$
R^{\frac{2(\gamma-1)}{2-\gamma}} v(R r ; a)=v\left(r ; R^{\frac{2(\gamma-1)}{2-\gamma}} a\right)
$$

In fact, the two sides of (4.43) solve the same ODE with the same initial data. We differentiate (4.43) with respect to $R$ and set $R=1, a=u_{0}(0)$ to get

$$
\frac{2(\gamma-1)}{2-\gamma} v\left(r ; u_{0}(0)\right)+r v^{\prime}\left(r ; u_{0}(0)\right)=u_{0}(0) \frac{2(\gamma-1)}{2-\gamma} v_{a}\left(r ; u_{0}(0)\right)
$$

Now taking the $r$ derivative and setting $r=1$, we get

$$
\begin{aligned}
u_{0}(0) \frac{2(\gamma-1)}{2-\gamma} v_{a}^{\prime}\left(1 ; u_{0}(0)\right) & =\frac{2(\gamma-1)}{2-\gamma} v^{\prime}\left(1 ; u_{0}(0)\right)+v^{\prime}\left(1 ; u_{0}(0)\right)+v^{\prime \prime}\left(1 ; u_{0}(0)\right) \\
& =\frac{2(\gamma-1)}{2-\gamma} v^{\prime}\left(1 ; u_{0}(0)\right)+v^{\prime}\left(1 ; u_{0}(0)\right)-2 v^{\prime}\left(1 ; u_{0}(0)\right) \\
& =\frac{3 \gamma-4}{2-\gamma} v^{\prime}\left(1 ; u_{0}(0)\right),
\end{aligned}
$$

where we used (4.42) to write $v^{\prime \prime}$ in terms of $v^{\prime}$. Since $v\left(r ; u_{0}(0)\right)=u_{0}(r)$, and $u_{0}(1)=0$, it follows that $v^{\prime}\left(1 ; u_{0}(0)\right)=u_{0}^{\prime}(1) \neq 0$. Noticing that $u_{0}(0) \neq 0, \frac{2(\gamma-1)}{2-\gamma} \neq 0$ for $\gamma \in\left(\frac{6}{5}, 2\right)$, we get $v_{a}^{\prime}\left(1 ; u_{0}(0)\right) \neq 0$ if and only if $\gamma \neq \frac{4}{3}$ from (4.45).

The case with condition (2.12) is a lot more difficult to prove, because we no longer have the scaling symmetry provided by an exact power law. We first restate (2.12) in terms of $h^{-1}$.

Lemma 4.6. 2.12) implies

$$
h^{-1}(s)<s\left(h^{-1}\right)^{\prime}(s) \leq 2 h^{-1}(s) \text { for } s>0 .
$$

Proof. Denote $h^{-1}(s)$ by $t$. (4.46) can be rewritten as

$$
t h^{\prime}(t)<h(t) \leq 2 t h^{\prime}(t),
$$

which is (2.12) by the definition of $h$ in terms of $p$.

To simplify equation, we make a change of variable $v(r)=\frac{w(r)}{r}$. If $v$ satisfies (4.21), then $w$ satisfies

$$
w^{\prime \prime}(r)+4 \pi g(w, r)=0, \quad w(0)=0, w^{\prime}(0)=a,
$$


where $g(w, r)=r h^{-1}\left(\frac{w}{r}\right)$. Denote the solution to (4.48) by $w(r ; a)$, then $v(r ; a)=\frac{w(r ; a)}{r}$, and $v_{a}^{\prime}\left(1 ; u_{0}(0)\right)=w_{a}^{\prime}\left(1 ; u_{0}(0)\right)-w_{a}\left(1 ; u_{0}(0)\right)$. By the proof of Lemma 4.2 this is equal to $-M^{\prime}\left(u_{0}(0)\right)$. Hence we are left to show $w_{a}^{\prime}\left(1 ; u_{0}(0)\right)-w_{a}\left(1 ; u_{0}(0)\right) \neq 0$.

To that end, we need the following Lemma, which extends Theorem 2.4 in 16 .

Lemma 4.7. Suppose $g: \mathbb{R} \times(0, \infty) \rightarrow \mathbb{R}$ is a $C^{1}$ map such that $g(w, r)>0$ when $w>0$ and $g(0, r)=0$ when $r>0$. Let $w(r ; a)$ be the solution to the initial value problem

$$
w^{\prime \prime}(r)+g(w, r)=0, \quad w(0)=0, w^{\prime}(0)=a,
$$

and $w\left(1 ; a_{0}\right)=0, w\left(r ; a_{0}\right)>0$ for $0<r<1$. In other words, $w\left(r ; a_{0}\right)$ is a positive solution to the boundary value problem

$$
w^{\prime \prime}(r)+g(w, r)=0, \quad w(0)=0, w(1)=0 .
$$

If we further assume the following conditions on $\mathrm{g}$ :

$$
\begin{gathered}
\lim _{r \rightarrow 0^{+}} g(w(r ; a), r)=0 \text { for a close to } a_{0} . \\
g-w g_{w}<0 \text { for } w>0,0<r \leq 1 . \\
g_{r} \leq 0 \text { for } w>0,0<r \leq 1 . \\
r g_{r}+3 g-w g_{w} \geq 0 \text { for } w>0,0<r \leq 1 .
\end{gathered}
$$

Then $w_{a}^{\prime}\left(1, a_{0}\right)-w_{a}\left(1, a_{0}\right)<0$.

Proof. We define several auxiliary functions. Let

$$
x(r ; a)=r w^{\prime}(r ; a), y(r ; a)=w^{\prime}(r ; a), z(r ; a)=w_{a}(r ; a) .
$$

One has

$$
\begin{gathered}
x\left(0^{+} ; a\right)=0, x^{\prime}\left(0^{+} ; a\right)=w^{\prime}(0 ; a)-\lim _{r \rightarrow 0^{+}} r g(w, r)=a . \\
y\left(0^{+} ; a\right)=a, y^{\prime}\left(0^{+} ; a\right)=-\lim _{r \rightarrow 0^{+}} g(w, r)=0 . \\
z\left(0^{+} ; a\right)=0, z^{\prime}\left(0^{+} ; a\right)=1 .
\end{gathered}
$$

We have

$$
\begin{aligned}
x^{\prime \prime} & =\left(r w^{\prime}\right)^{\prime \prime} \\
& =r w^{\prime \prime \prime}+2 w^{\prime \prime} \\
& =r\left(-g_{r}-g_{w} w^{\prime}\right)-2 g \\
& =-r g_{r}-g_{w} x-2 g .
\end{aligned}
$$

Here we used (4.50). Hence $x$ satisfies the equation

$$
x^{\prime \prime}+g_{w} x+r g_{r}+2 g=0 .
$$

Similarly $y$ satisfies the equation

$$
y^{\prime \prime}+g_{w} y+g_{r}=0 .
$$

$z$ satisfies

$$
z^{\prime \prime}+g_{w} z=0 .
$$

From these we get the ODEs of various Wronskians between $w, x, y$ and $z$ :

$$
\begin{gathered}
W(x, z)^{\prime}=\left|\begin{array}{cc}
x & z \\
x^{\prime} & z^{\prime}
\end{array}\right|^{\prime}=z\left(r g_{r}+2 g\right) . \\
W(y, z)^{\prime}=\left|\begin{array}{cc}
y & z \\
y^{\prime} & z^{\prime}
\end{array}\right|^{\prime}=z g_{r} .
\end{gathered}
$$




$$
W(w, z)^{\prime}=\left|\begin{array}{cc}
w & z \\
w^{\prime} & z^{\prime}
\end{array}\right|^{\prime}=z\left(g-w g_{w}\right)
$$

In the following, we set $a$ equal to $a_{0}$ in all functions. Since $w>0$ and $w^{\prime \prime}=-g<0$ for $r \in(0,1)$, we know that $w$ is a positive convex function with a unique maximum and zero boundary value on $[0,1]$. By (4.58),$z(r)>0$ for $r$ close to 0 . We claim that there is an $r_{0} \in(0,1)$ such that $z\left(r_{0}\right)=0$. If not, then $z(r)>0$ for all $r \in(0,1)$. Integrating (4.65) on $(0,1)$ and using the boundary conditions of $w$ and $z$, we have

$$
-w^{\prime}(1) z(1)=\int_{0}^{1} z\left(g-w g_{w}\right) d r<0 .
$$

The inequality is a consequence of (4.52). However, we know that $w^{\prime}(1)<0$ and $z(1) \geq 0$, making the left hand side of (4.66) non-negative. Such a contradiction implies the existence of $r_{0}$ mentioned above. We assume $r_{0}$ is the smallest value in $(0,1)$ for which $z\left(r_{0}\right)=0$. (4.52) and (4.54) imply $r g_{r}+2 g>0$. If we now integrate (4.63) on $\left(0, r_{0}\right)$,

$$
x\left(r_{0}\right) z^{\prime}\left(r_{0}\right)=\int_{0}^{r_{0}} z\left(r g_{r}+2 g\right) d r>0
$$

The last inequality follows from the fact that $z(r)>0$ for $r \in\left(0, r_{0}\right)$. Since $z^{\prime}\left(r_{0}\right)<0$, we have $x\left(r_{0}\right)<0$, which also implies that $w^{\prime}\left(r_{0}\right)<0$.

Our next claim is that $z(r)<0$ for all $r_{0}<r \leq 1$. If not, let $r_{1} \in\left(r_{0}, 1\right]$ be the first zero of $z$ strictly bigger than $r_{0}$. Integrating (4.64) on $\left(r_{0}, r_{1}\right)$ and recalling the definition of $y$, we obtain

$$
w^{\prime}\left(r_{1}\right) z^{\prime}\left(r_{1}\right)-w^{\prime}\left(r_{0}\right) z^{\prime}\left(r_{0}\right)=y\left(r_{1}\right) z^{\prime}\left(r_{1}\right)-y\left(r_{0}\right) z^{\prime}\left(r_{0}\right)=\int_{r_{0}}^{r_{1}} z g_{r} d r \geq 0 .
$$

The last inequality follows from (4.53) and the fact that $z(r)<0$ for $r \in\left(r_{0}, r_{1}\right)$. However, the fact that $w^{\prime}\left(r_{0}\right)<0$ implies $w^{\prime}\left(r_{1}\right)<0$. We also have $z^{\prime}\left(r_{0}\right)<0$, and $z^{\prime}\left(r_{1}\right)>0$. These conditions together imply that the left hand side of (4.68) is negative. Such a contradiction implies $z(r)<0$ for all $r_{0}<r \leq 1$.

We integrate $W(x, z)^{\prime}+r_{0} W(w, z)^{\prime}$ between 0 and $r_{0}$, and integrate $W(y, z)^{\prime}+W(w, z)^{\prime}$ between $r_{0}$ and 1 to get

$$
\begin{gathered}
r_{0}\left[w^{\prime}\left(r_{0}\right)+w\left(r_{0}\right)\right] z^{\prime}\left(r_{0}\right)=\int_{0}^{r_{0}} z\left(r g_{r}+2 g+r_{0} g-r_{0} w g_{w}\right) d r . \\
w^{\prime}(1)\left[z^{\prime}(1)-z(1)\right]=z^{\prime}\left(r_{0}\right)\left(w^{\prime}\left(r_{0}\right)+w\left(r_{0}\right)\right)+\int_{r_{0}}^{1} z\left(g_{r}+g-w g_{w}\right) d r .
\end{gathered}
$$

Notice (4.52) and (4.53) imply $r g_{r}+2 g+r_{0} g-r_{0} w g_{w} \geq 0$ for all $r_{0} \in(0,1)$. This together with the fact that $z(r)>0$ for $r \in\left(0, r_{0}\right)$ imply that (4.69) is non-negative. Since $r_{0} \in(0,1)$, it follows that the first term on the right hand side of (4.70) is non-negative. But the second term on the right hand side of (4.70) is positive by (4.52), (4.53), and the fact that $z(r)<0$ for $r \in\left(r_{0}, 1\right)$. Therefore (4.70) is positive. Since $w^{\prime}(1)<0$ as is explained above, we get $z^{\prime}(1)-z(1)<0$, which is the conclusion of the lemma by the definition of $z$.

To apply Lemma 4.6, we verify the conditions on

$$
g(w, r)=4 \pi r h^{-1}\left(\frac{w}{r}\right) .
$$

Condition (4.52) reads:

$$
r h^{-1}\left(\frac{w}{r}\right)-w\left(h^{-1}\right)^{\prime}\left(\frac{w}{r}\right)<0 \text { for } w>0 \text { and } 0<r \leq 1,
$$

which is a consequence of

$$
s\left(h^{-1}\right)^{\prime}(s)>h^{-1}(s) \text { for } s>0 .
$$


This is included in (4.46) in Lemma 4.6. Condition (4.53) reads:

$$
h^{-1}\left(\frac{w}{r}\right)-\frac{w}{r}\left(h^{-1}\right)^{\prime}\left(\frac{w}{r}\right) \leq 0,
$$

which is implied by (4.73). Condition (4.54) reads

$$
h^{-1}\left(\frac{w}{r}\right)-\frac{w}{r}\left(h^{-1}\right)^{\prime}\left(\frac{w}{r}\right)+3 h^{-1}\left(\frac{w}{r}\right)-\frac{w}{r}\left(h^{-1}\right)^{\prime}\left(\frac{w}{r}\right) \geq 0
$$

or

$$
2 h^{-1}\left(\frac{w}{r}\right)-\frac{w}{r}\left(h^{-1}\right)^{\prime}\left(\frac{w}{r}\right) \geq 0 .
$$

This is a consequence of

$$
s\left(h^{-1}\right)^{\prime}(s) \leq 2 h^{-1}(s) \text { for } s>0,
$$

which is included in (4.46). By the discussion above Lemma 4.7 it implies the second case in Theorem 2.2 .

4.6. Example: oblateness for constant rotation. In this subsection, we exhibit the approximate support of $\rho_{\kappa}$ as constructed in Theorem 2.1 when $\kappa$ is small in case the rotation profile $\omega$ is constant. In particular, the approximation shows that the support of $\rho_{\kappa}$ is wider at the equator than at the poles. Thus the body has an oblate shape.

As is explained in Section 2.3, $\rho_{\kappa}$ is constructed by deforming the ball using $g_{\zeta_{\kappa}}$. Therefore the boundary of the support of $\rho_{\kappa}$ is precisely $g_{\zeta_{\kappa}}\left(\partial B_{1}\right)=\left\{x\left(1+\zeta_{\kappa}(x)\right): x \in \partial B_{1}\right\}$. We just need to find out the value of $\zeta_{\kappa}$ on the boundary. If the conditions of Theorem 2.1 are satisfied, we expand $\zeta_{\kappa}$ near $\kappa=0$ and use the implicit function theorem to evaluate the first derivative:

$$
\zeta_{\kappa}=-\kappa\left(\frac{\partial \mathcal{F}}{\partial \zeta}(0,0)\right)^{-1} \frac{\partial \mathcal{F}}{\partial \kappa}(0,0)+R(\kappa)
$$

where $\|R(\kappa)\|_{X}=o(\kappa)$ as $\kappa \rightarrow 0$. We now study the dominant term. By (2.22) and (2.13),

$$
\frac{\partial \mathcal{F}}{\partial \kappa}(0,0)=\int_{0}^{r(x)} \omega^{2}(s) s d s=\frac{1}{2} r^{2}(x)=\frac{1}{2} r^{2} \sin ^{2} \theta_{1}=r^{2}\left(\frac{2}{3} \sqrt{\pi} Y_{00}-\frac{2}{3} \sqrt{\frac{\pi}{5}} Y_{20}\right) .
$$

Here we assume $\omega(r)=1$ as an example of a constant rotation profile, and used spherical coordinates $\left(r, \theta_{1}, \theta_{2}\right)$ to write the last expression. The spherical harmonics $Y_{00}$ and $Y_{20}$ are

$$
Y_{00}\left(\theta_{1}, \theta_{2}\right)=\frac{1}{2} \sqrt{\frac{1}{\pi}}, \quad Y_{20}\left(\theta_{1}, \theta_{2}\right)=\frac{1}{4} \sqrt{\frac{5}{\pi}}\left(3 \cos ^{2} \theta_{1}-1\right) .
$$

Using the expression for $\frac{\partial \mathcal{F}}{\partial \zeta}(0,0)$ given in (4.8), we have

$$
-\left(\frac{\partial \mathcal{F}}{\partial \zeta}(0,0)\right)^{-1} \frac{\partial \mathcal{F}}{\partial \kappa}(0,0)=\xi
$$

where $\xi \in X$ is the unique solution to the equation

$$
\frac{u_{0}^{\prime}(x)}{|x|} \xi(x)-\int_{B_{1}} \frac{\rho_{0}^{\prime}(y)}{|y|} \xi(y)\left(\frac{1}{|x-y|}-\frac{1}{|y|}\right) d y+\frac{k\left(\rho_{0}\right)(x)-k\left(\rho_{0}\right)(0)}{M} \int_{B_{1}} \frac{\rho_{0}^{\prime}(y)}{|y|} \xi(y) d y
$$

$$
=r^{2}\left(-\frac{2}{3} \sqrt{\pi} Y_{00}+\frac{2}{3} \sqrt{\frac{\pi}{5}} Y_{20}\right) .
$$

The existence and uniqueness of $\xi$ follows from the fact that $\frac{\partial \mathcal{F}}{\partial \zeta}(0,0)$ is an isomorphism, as is established in Section 4.4. Since we are only interested in the deviation from spherical symmetry of the support of $\rho_{\kappa}$, we pay attention only to the non-radial components of $\xi$. We proceed in a fashion parallel to Section 4.3. Defining $\varphi$ as in (4.30) and taking the Laplacian of $\varphi$, we again arrive at (4.31). Projecting onto spherical harmonics $Y_{l m}$ for $l \geq 1$ as before, 
we get (4.32) so long as $(l, m) \neq(2,0)$. The same argument as in section 4.3 shows that $\varphi_{l m}=0$ for $(l, m) \neq(2,0)$. However, for $(l, m)=(2,0)$, we get

$$
\Delta \varphi_{20}-\frac{6}{r^{2}} \varphi_{20}= \begin{cases}-4 \pi \frac{\rho_{0}^{\prime}}{|x|} \xi_{20} & \text { if }|x| \leq 1, \\ 0 & \text { if }|x|>1 .\end{cases}
$$

Projecting (4.82) onto $Y_{20}$, we get

$$
\frac{u_{0}^{\prime}}{|x|} \xi_{20}-\varphi_{20}=\frac{2}{3} \sqrt{\frac{\pi}{5}} r^{2} .
$$

Hence

$$
\Delta \varphi_{20}-\frac{6}{r^{2}} \varphi_{20}= \begin{cases}-4 \pi \frac{\rho_{0}^{\prime}}{u_{0}^{\prime}}\left(\varphi_{20}+\frac{2}{3} \sqrt{\frac{\pi}{5}} r^{2}\right) & \text { if }|x| \leq 1, \\ 0 & \text { if }|x|>1 .\end{cases}
$$

As before, we deduce $\varphi_{20}=\frac{C}{r^{3}}$ for $r \geq 1$. For $r<1$, the function $\psi_{20}=\frac{\varphi_{20}}{u_{0}^{\prime}}$ satisfies

$$
\Delta \psi_{20}+2 \frac{u_{0}^{\prime \prime}}{u_{0}^{\prime}} \psi_{20}^{\prime}-\frac{4}{r^{2}} \psi_{20}=-4 \pi \frac{\rho_{0}^{\prime}}{\left(u_{0}^{\prime}\right)^{2}} \frac{2}{3} \sqrt{\frac{\pi}{5}} r^{2}>0
$$

for $0<r<1$. The same maximum principle argument as in Section 4.3 yields $\psi_{20}(r) \leq 0$ for $0<r \leq 1$. Since $u_{0}^{\prime}<0$ for $0<r \leq 1$ by Lemma 3.2. $\varphi_{20}(r) \geq 0$ for $0<r \leq 1$. By (4.84),

$$
\xi_{20}(1)=\frac{1}{u_{0}^{\prime}(1)}\left(\varphi_{20}(1)+\frac{2}{3} \sqrt{\frac{\pi}{5}}\right) \leq \frac{1}{u_{0}^{\prime}(1)} \frac{2}{3} \sqrt{\frac{\pi}{5}}<0 .
$$

The non-radial component of $\xi$ at $\partial B_{1}$ is given by

$$
\xi_{20}(1) Y_{20}\left(\theta_{1}, \theta_{2}\right)=\left|\xi_{20}(1)\right| \frac{1}{4} \sqrt{\frac{5}{\pi}}\left(1-3 \cos ^{2} \theta_{1}\right)
$$

Of course, the poles correspond to $\theta_{1}=0, \pi$ and the equator to $\theta_{1}=\frac{\pi}{2}$. This means $\xi$ is larger at the equator than at the poles. In view of (4.78) and (4.81), we have proved

Theorem 4.2. If the conditions of Theorem 2.1 are satisfied, and the angular velocity profile $\omega(r)$ is constant, then for $\kappa$ sufficiently small, the support of $\rho_{\kappa}$ is larger at the equator than at the poles.

\section{Euler Model: Fréchet differentiability}

In this section, we prove the Fréchet differentiability of $\mathcal{F}(\zeta, \kappa)$ under the assumptions of Theorem 2.1

Theorem 5.1. The operator $\mathcal{F}: B_{\epsilon}(X) \times \mathbb{R} \rightarrow X$ with $\epsilon>0$ sufficiently small is continuously Fréchet differentiable with $\frac{\partial \mathcal{F}}{\partial \zeta}$ given by (5.17) - (5.21).

We will prove this theorem step by step by means of the following lemmas. We write $\mathcal{F}$ as

$$
\mathcal{F}(\zeta, \kappa)=\mathcal{M}(\zeta) \mathcal{F}_{1}(\zeta)+\kappa \mathcal{F}_{2}(\zeta)+\mathcal{F}_{3}(\zeta)
$$

where

$$
\begin{aligned}
\mathcal{F}_{1}(\zeta)(x) & =\int_{g_{\zeta}\left(B_{1}\right)} \rho_{0}\left(g_{\zeta}^{-1}\left(y^{\prime}\right)\right)\left(\frac{1}{\left|g_{\zeta}(x)-y^{\prime}\right|}-\frac{1}{\left|y^{\prime}\right|}\right) d y^{\prime} \\
& =\int_{B_{2}} \rho_{0}\left(g_{\zeta}^{-1}\left(y^{\prime}\right)\right)\left(\frac{1}{\left|g_{\zeta}(x)-y^{\prime}\right|}-\frac{1}{\left|y^{\prime}\right|}\right) d y^{\prime} .
\end{aligned}
$$

The last equality follows from the fact that $\rho_{0}$ is supported on $\overline{B_{1}}$ and that $g_{\zeta}\left(\overline{B_{1}}\right) \subset B_{2}$.

$$
\mathcal{F}_{2}(\zeta)(x)=\int_{0}^{r\left(g_{\zeta}(x)\right)} \omega^{2}(s) s d s, \quad r\left(g_{\zeta}(x)\right)=\left(1+\frac{\zeta(x)}{|x|^{2}}\right) \sqrt{x_{1}^{2}+x_{2}^{2}},
$$


and

$$
\mathcal{F}_{3}(\zeta)(x)=-h\left(\mathcal{M}(\zeta) \rho_{0}(x)\right)+h\left(\mathcal{M}(\zeta) \rho_{0}(0)\right) .
$$

First of all, we want $\mathcal{F}$ to map $B_{\epsilon}(X) \times \mathbb{R}$ into $X$. It is easy to see that $\mathcal{F}(\zeta, \omega)$ has the symmetry requirements of $X$.

Lemma 5.1. There is a constant $C>0$ depending on $\rho_{0}$ and $\epsilon$ such that

$$
\|\mathcal{F}(\zeta, \kappa)\|_{X} \leq C(1+\kappa)
$$

if $\zeta \in B_{\epsilon}(X)$ for $\epsilon$ small enough.

Proof. To estimate the norm, we calculate the spatial derivatives of $\mathcal{F}(\zeta, \kappa)$.

$$
\partial_{i} \mathcal{F}(\zeta, \kappa)(x)=\mathcal{M}(\zeta) \partial_{i} \mathcal{F}_{1}(\zeta)(x)+\kappa \partial_{i} \mathcal{F}_{2}(\zeta)(x)+\partial_{i} \mathcal{F}_{3}(\zeta)(x)
$$

We consider the three terms separately. Note that

$$
|\mathcal{M}(\zeta)| \leq \frac{1}{1-C\|\zeta\|_{X}} \leq 2
$$

if $\|\zeta\|_{X}<\epsilon$ is small enough, by (2.17) and (3.18). By Lemma 3.6 (3.16) and (3.20),

$$
\partial_{i} \mathcal{F}_{1}(\zeta)(x)=\left(\int_{B_{2}} \rho_{0}\left(g_{\zeta}^{-1}\left(y^{\prime}\right)\right) \frac{-\left(g_{\zeta}(x)-y^{\prime}\right)}{\left|g_{\zeta}(x)-y^{\prime}\right|^{3}} d y^{\prime}\right) \cdot \partial_{i} g_{\zeta}(x)
$$

satisfies

$$
\begin{aligned}
\left|\partial_{i} \mathcal{F}_{1}(\zeta)(x)\right| & =\left|V_{\rho_{0}\left(g_{\zeta}^{-1}(\cdot)\right)}\left(g_{\zeta}(x)\right) \cdot \partial_{i} g_{\zeta}(x)\right| \\
& \leq C\left(\left\|\rho_{0}\right\|_{C^{1}\left(B_{1 / 2}\right)}+\left\|\rho_{0}\right\|_{C^{0}\left(B_{1}\right)}\right)\left(1+\|\zeta\|_{X}\right)|x|
\end{aligned}
$$

for all $x \in \overline{B_{1}}$. Next, notice from (3.24) that

$$
\begin{gathered}
\partial_{i}\left[r\left(g_{\zeta}(x)\right)\right]=\partial_{i}\left(\frac{\zeta(x)}{|x|^{2}}\right) \sqrt{x_{1}^{2}+x_{2}^{2}}+\left(1+\frac{\zeta(x)}{|x|^{2}}\right) \frac{x_{1} \delta_{i 1}+x_{2} \delta_{i 2}}{\sqrt{x_{1}^{2}+x_{2}^{2}}}, \\
\left|r\left(g_{\zeta}(x)\right)\right| \leq C|x|, \quad\left|\partial_{i}\left[r\left(g_{\zeta}(x)\right)\right]\right| \leq C .
\end{gathered}
$$

Hence

$$
\left|\partial_{i} \mathcal{F}_{2}(\zeta)(x)\right|=\omega^{2}\left[r\left(g_{\zeta}(x)\right)\right] r\left(g_{\zeta}(x)\right)\left|\partial_{i}\left[r\left(g_{\zeta}(x)\right)\right]\right| \leq C|x|
$$

since $\omega^{2}$ is locally bounded. To estimate $\mathcal{F}_{3}$, we recall that by $(3.15)$, we have

$$
\partial_{i} \mathcal{F}_{3}(\zeta)(x)=-h^{\prime}\left(\mathcal{M}(\zeta) \rho_{0}(x)\right) \mathcal{M}(\zeta) \partial_{i} \rho_{0}(x)=-\mathcal{M}(\zeta) \frac{h^{\prime}\left(\mathcal{M}(\zeta) \rho_{0}(x)\right)}{h^{\prime}\left(\rho_{0}(x)\right)} \partial_{i} u_{0}(x) .
$$

By (3.2), we have

$$
\lim _{s \rightarrow 0^{+}} \frac{h^{\prime}(\mathcal{M} s)}{h^{\prime}(s)}=\mathcal{M}^{\gamma-2} .
$$

Together with the regularity of $u_{0}$ given in Lemma 3.2, this implies that (5.13) is continuous on $\overline{B_{1}}$. Furthermore it is easy to see that $\left|\partial_{i} \mathcal{F}_{3}(\zeta)(x)\right| \leq C|x|$ because $u_{0} \in C^{2}\left(\overline{B_{1}}\right)$ and $\partial_{i} u_{0}(0)=0$. This completes the proof.

We now compute the formal derivative of $\mathcal{F}$ with respect to $\zeta$, which we denote by $\mathcal{F}^{\prime}(\zeta, \kappa)$. In order to facilitate future estimates of the formal derivative, we introduce the new notation

$$
F(x, s)=\mathcal{F}(\zeta+s \xi, \kappa)(x)
$$

where $\zeta \in B_{\epsilon}(X), \xi \in X$ are chosen, and $s$ is restricted to a sufficiently small neighborhood of 0 so that $\zeta+s \xi \in B_{\epsilon}(X)$. We define the formal derivative of $\mathcal{F}$ with respect to $\zeta$ as the pointwise limit

$$
\left[\mathcal{F}^{\prime}(\zeta, \kappa) \xi\right](x)=\left(\partial_{s} F\right)(x, 0)=\left.\partial_{s}\right|_{s=0} \mathcal{F}(\zeta+s \xi, \kappa)(x)
$$


for every fixed $x$.

Lemma 5.2. The formal derivative is

$$
\mathcal{F}^{\prime}(\zeta, \kappa) \xi=\left[\mathcal{M}^{\prime}(\zeta) \xi\right] \mathcal{F}_{1}(\zeta)+\mathcal{M}(\zeta) \mathcal{F}_{1}^{\prime}(\zeta) \xi+\kappa \mathcal{F}_{2}^{\prime}(\zeta) \xi+\mathcal{F}_{3}^{\prime}(\zeta) \xi
$$

where $\mathcal{M}^{\prime}(\zeta) \xi=$

$$
\frac{-M}{\left(\int_{B_{1}} \rho_{0}(x) \operatorname{det} D g_{\zeta}(x) d x\right)^{2}} \int_{B_{1}} \rho_{0}(x) \operatorname{det} D g_{\zeta}(x) \operatorname{tr}\left[\left(D g_{\zeta}\right)^{-1}(x) D\left(\xi(x) \frac{x}{|x|^{2}}\right)\right] d x,
$$

and $\left[\mathcal{F}_{1}^{\prime}(\zeta) \xi\right](x)=$

$$
\begin{gathered}
\int_{B_{2}}[- \\
+\rho_{0}\left(g_{\zeta}^{-1}\left(y^{\prime}\right)\right)\left(D g_{\zeta}\right)^{-1}\left(y^{\prime}\right) \xi\left(g_{\zeta}^{-1}\left(y^{\prime}\right)\right) \frac{g_{\zeta}^{-1}\left(y^{\prime}\right)}{\left|g_{\zeta}^{-1}\left(y^{\prime}\right)\right|^{2}}\left(\frac{1}{\left|g_{\zeta}(x)-y^{\prime}\right|}-\frac{1}{\left|y^{\prime}\right|}\right) \\
\left.+\rho_{0}\left(g_{\zeta}^{-1}\left(y^{\prime}\right)\right) \frac{-\left(g_{\zeta}(x)-y^{\prime}\right)}{\left|g_{\zeta}(x)-y^{\prime}\right|^{3}} \cdot \xi(x) \frac{x}{|x|^{2}}\right] d y^{\prime} \\
{\left[\mathcal{F}_{2}^{\prime}(\zeta) \xi\right](x)=\omega^{2}\left[r\left(g_{\zeta}(x)\right)\right] r\left(g_{\zeta}(x)\right) \frac{\xi(x)}{|x|^{2}} \sqrt{x_{1}^{2}+x_{2}^{2}} .} \\
{\left[\mathcal{F}_{3}^{\prime}(\zeta) \xi\right](x)=\left[-h^{\prime}\left(\mathcal{M}(\zeta) \rho_{0}(x)\right) \rho_{0}(x)+h^{\prime}\left(\mathcal{M}(\zeta) \rho_{0}(0)\right) \rho_{0}(0)\right] \mathcal{M}^{\prime}(\zeta) \xi}
\end{gathered}
$$

Proof. Note that $\left(D g_{\zeta}\right)^{-1}$ denote the inverse matrix of $D g_{\zeta}$. (5.17) is obvious. We compute

$$
\begin{aligned}
& \mathcal{M}^{\prime}(\zeta) \xi=\left.\partial_{s}\right|_{s=0} \mathcal{M}(\zeta+s \xi) \\
= & \left.\frac{-M}{\left(\int_{B_{1}} \rho_{0}(x) \operatorname{det} D g_{\zeta}(x) d x\right)^{2}} \partial_{s}\right|_{s=0} \int_{B_{1}} \rho_{0}(x) \operatorname{det} D g_{\zeta+s \xi}(x) d x \\
= & \frac{-M}{\left(\int_{B_{1}} \rho_{0}(x) \operatorname{det} D g_{\zeta}(x) d x\right)^{2}} \int_{B_{1}} \rho_{0}(x) \operatorname{det} D g_{\zeta}(x) \operatorname{tr}\left[\left.D g_{\zeta}^{-1} \partial_{s}\right|_{s=0} D g_{\zeta+s \xi}\right](x) d x \\
= & \frac{-M}{\left(\int_{B_{1}} \rho_{0}(x) \operatorname{det} D g_{\zeta}(x) d x\right)^{2}} \int_{B_{1}} \rho_{0}(x) \operatorname{det} D g_{\zeta}(x) \operatorname{tr}\left[D g_{\zeta}^{-1}(x) D\left(\xi(x) \frac{x}{|x|^{2}}\right)\right] d x
\end{aligned}
$$

Differentiation under the integral sign is justified by dominated convergence. We use

$$
\frac{d(\operatorname{det} F)}{d s}=(\operatorname{det} F) \operatorname{tr}\left(F^{-1} \frac{d F}{d s}\right), \quad \frac{d}{d s} D g_{\zeta+s \xi}=D\left(\xi(x) \frac{x}{|x|^{2}}\right)
$$

to obtain (5.18). Next

$$
\left[\mathcal{F}_{1}^{\prime}(\zeta) \xi\right](x)=\left.\partial_{s}\right|_{s=0} \int_{B_{2}} \rho_{0}\left(g_{\zeta+s \xi}^{-1}\left(y^{\prime}\right)\right)\left(\frac{1}{\left|g_{\zeta+s \xi}(x)-y^{\prime}\right|}-\frac{1}{\left|y^{\prime}\right|}\right) d y^{\prime} .
$$

To carry out the derivative we split the integral into two terms

$$
I_{1}(s)=\int_{B_{2}} \rho_{0}\left(g_{\zeta+s \xi}^{-1}\left(y^{\prime}\right)\right) \frac{1}{\left|g_{\zeta+s \xi}(x)-y^{\prime}\right|} d y^{\prime}, \quad I_{2}(s)=\int_{B_{2}} \rho_{0}\left(g_{\zeta+s \xi}^{-1}\left(y^{\prime}\right)\right) \frac{1}{\left|y^{\prime}\right|} d y^{\prime},
$$

where $s$ belongs to a small neighborhood of 0 . We use a cutoff function to avoid the singularity. Let $\chi$ be a non-negative, smooth, compactly supported function on the real line such that $\chi(s)=1$ for $|s|<1, \chi(s)=0$ for $|s|>2$, and $\left\|\chi^{\prime}\right\|_{\infty} \leq 2$. Let

$$
I_{1, \epsilon}(s)=\int_{B_{2}} \rho_{0}\left(g_{\zeta+s \xi}^{-1}\left(y^{\prime}\right)\right) \frac{1}{\left|g_{\zeta+s \xi}(x)-y^{\prime}\right|}\left[1-\chi\left(\frac{\left|g_{\zeta+s \xi}(x)-y^{\prime}\right|}{\epsilon}\right)\right] d y^{\prime}
$$


We see easily that $I_{1, \epsilon}(s)$ converges uniformly to $I_{1}(s)$ as $\epsilon \rightarrow 0$, and that $I_{1, \epsilon}(s)$ is $C^{1}$ with

$$
\begin{aligned}
I_{1, \epsilon}^{\prime}(s)= & \int_{B_{2}} \partial_{s}\left(\rho_{0}\left(g_{\zeta+s \xi}^{-1}\left(y^{\prime}\right)\right) \frac{1}{\left|g_{\zeta+s \xi}(x)-y^{\prime}\right|}\right)\left[1-\chi\left(\frac{\left|g_{\zeta+s \xi}(x)-y^{\prime}\right|}{\epsilon}\right)\right] d y^{\prime} \\
& -\int_{B_{2}} \rho_{0}\left(g_{\zeta+s \xi}^{-1}\left(y^{\prime}\right)\right) \frac{1}{\left|g_{\zeta+s \xi}(x)-y^{\prime}\right|} \partial_{s} \chi\left(\frac{\left|g_{\zeta+s \xi}(x)-y^{\prime}\right|}{\epsilon}\right) d y^{\prime} .
\end{aligned}
$$

Now

$$
\begin{aligned}
& \left|\partial_{s}\left(\rho_{0}\left(g_{\zeta+s \xi}^{-1}\left(y^{\prime}\right)\right) \frac{1}{\left|g_{\zeta+s \xi}(x)-y^{\prime}\right|}\right)\right| \\
= & \mid-\nabla \rho_{0}\left(g_{\zeta+s \xi}^{-1}\left(y^{\prime}\right)\right) D g_{\zeta+s \xi}^{-1}\left(y^{\prime}\right) \xi\left(g_{\zeta+s \xi}^{-1}\left(y^{\prime}\right)\right) \frac{g_{\zeta+s \xi}^{-1}\left(y^{\prime}\right)}{\left|g_{\zeta+s \xi}^{-1}\left(y^{\prime}\right)\right|^{2}} \frac{1}{\left|g_{\zeta+s \xi}(x)-y^{\prime}\right|} \\
& +\rho_{0}\left(g_{\zeta+s \xi}^{-1}\left(y^{\prime}\right)\right) \frac{-\left(g_{\zeta+s \xi}(x)-y^{\prime}\right)}{\left|g_{\zeta+s \xi}(x)-y^{\prime}\right|^{3}} \cdot \xi(x) \frac{x}{|x|^{2}} \mid \leq C \frac{1}{\left|g_{\zeta+s \xi}(x)-y^{\prime}\right|^{2}} .
\end{aligned}
$$

In the above calculation, we have used the formula

$$
\partial_{s} g_{\zeta+s \xi}^{-1}\left(y^{\prime}\right)=-D g_{\zeta+s \xi}^{-1}\left(y^{\prime}\right) \xi\left(g_{\zeta+s \xi}^{-1}\left(y^{\prime}\right)\right) \frac{g_{\zeta+s \xi}^{-1}\left(y^{\prime}\right)}{\left|g_{\zeta+s \xi}^{-1}\left(y^{\prime}\right)\right|^{2}},
$$

which follows if we differentiate $g_{\zeta+s \xi}\left(g_{\zeta+s \xi}^{-1}\left(y^{\prime}\right)\right)=y^{\prime}$ with respect to $s$. In addition,

$$
\left|\rho_{0}\left(g_{\zeta+s \xi}^{-1}\left(y^{\prime}\right)\right) \frac{1}{\left|g_{\zeta+s \xi}(x)-y^{\prime}\right|}\right| \leq C \frac{1}{\left|g_{\zeta+s \xi}(x)-y^{\prime}\right|}
$$

Also

$$
\begin{aligned}
\left|\partial_{s} \chi\left(\frac{\left|g_{\zeta+s \xi}(x)-y^{\prime}\right|}{\epsilon}\right)\right| & =\left|\chi^{\prime}\left(\frac{\left|g_{\zeta+s \xi}(x)-y^{\prime}\right|}{\epsilon}\right) \frac{g_{\zeta+s \xi}(x)-y^{\prime}}{\epsilon\left|g_{\zeta+s \xi}(x)-y^{\prime}\right|} \cdot \xi(x) \frac{x}{|x|^{2}}\right| \\
& \leq \frac{C}{\epsilon}\left|\chi^{\prime}\left(\frac{\left|g_{\zeta+s \xi}(x)-y^{\prime}\right|}{\epsilon}\right)\right|
\end{aligned}
$$

so that that $I_{1, \epsilon}^{\prime}(s)$ converges uniformly to

$$
\int_{B_{2}} \partial_{s}\left(\rho_{0}\left(g_{\zeta+s \xi}^{-1}\left(y^{\prime}\right)\right) \frac{1}{\left|g_{\zeta+s \xi}(x)-y^{\prime}\right|}\right) d y^{\prime}
$$

as $\epsilon \rightarrow 0$. Therefore $I_{1}(s)$ is $C^{1}$ with derivative given by (5.33). The calculation of $I_{2}^{\prime}(s)$ as well as of $\mathcal{F}_{2}^{\prime}$ and $\mathcal{F}_{3}^{\prime}$ is similar.

Before we prove that the formal derivative $\mathcal{F}^{\prime}(\zeta, \kappa)$ computed in Lemma 5.2 is really a Fréchet derivative, we will show that it is a bounded linear map on $X$, and that the dependence of $\mathcal{F}^{\prime}$ on $\zeta$ is continuous. To that end, we compute the spatial derivatives of $\mathcal{F}^{\prime}(\zeta, \kappa) \xi$.

\section{Lemma 5.3.}

$$
\begin{aligned}
& \partial_{i}\left[\mathcal{F}^{\prime}(\zeta, \kappa) \xi\right](x) \\
= & {\left[\mathcal{M}^{\prime}(\zeta) \xi\right] \partial_{i}\left[\mathcal{F}_{1}(\zeta)\right](x)+\mathcal{M}(\zeta) \partial_{i}\left[\mathcal{F}_{1}^{\prime}(\zeta) \xi\right](x)+\kappa \partial_{i}\left[\mathcal{F}_{2}^{\prime}(\zeta) \xi\right](x)+\partial_{i}\left[\mathcal{F}_{3}^{\prime}(\zeta) \xi\right](x), }
\end{aligned}
$$


where $\partial_{i}\left[\mathcal{F}_{1}(\zeta)\right](x)$ is given in (5.8),

$$
\begin{aligned}
& \partial_{i}\left[\mathcal{F}_{1}^{\prime}(\zeta) \xi\right](x) \\
& =\int_{B_{2}}-\left(\nabla \rho_{0}\left(g_{\zeta}^{-1}\left(y^{\prime}\right)\right) D g_{\zeta}^{-1}\left(y^{\prime}\right) \xi\left(g_{\zeta}^{-1}\left(y^{\prime}\right)\right) \frac{g_{\zeta}^{-1}\left(y^{\prime}\right)}{\left|g_{\zeta}^{-1}\left(y^{\prime}\right)\right|^{2}}\right) \frac{-\left(g_{\zeta}(x)-y^{\prime}\right)}{\left|g_{\zeta}(x)-y^{\prime}\right|^{3}} d y^{\prime} \cdot \partial_{i} g_{\zeta}(x) \\
& +\sum_{j} \int_{B_{2}}\left(\nabla \rho_{0}\left(g_{\zeta}^{-1}\left(y^{\prime}\right)\right) \partial_{j} g_{\zeta}^{-1}\left(y^{\prime}\right)\right)\left(\frac{-\left(g_{\zeta}(x)-y^{\prime}\right)}{\left|g_{\zeta}(x)-y^{\prime}\right|^{3}} \cdot \partial_{i} g_{\zeta}(x)\right) d y^{\prime} \xi(x) \frac{x_{j}}{|x|^{2}} \\
& +\int_{B_{2}} \rho_{0}\left(g_{\zeta}^{-1}\left(y^{\prime}\right)\right) \frac{-\left(g_{\zeta}(x)-y^{\prime}\right)}{\left|g_{\zeta}(x)-y^{\prime}\right|^{3}} d y^{\prime} \cdot \partial_{i}\left(\xi(x) \frac{x}{|x|^{2}}\right), \\
& \partial_{i}\left[\mathcal{F}_{2}^{\prime}(\zeta) \xi\right](x)=\left(\omega^{2}\right)^{\prime}\left[r\left(g_{\zeta}(x)\right)\right] \partial_{i}\left[r\left(g_{\zeta}(x)\right)\right] r\left(g_{\zeta}(x)\right) \frac{\xi(x)}{|x|^{2}} \sqrt{x_{1}^{2}+x_{2}^{2}} \\
& \quad+\omega^{2}\left(r\left(g_{\zeta}(x)\right)\right)\left[\partial_{i}\left[r\left(g_{\zeta}(x)\right)\right] \frac{\xi(x)}{|x|^{2}} \sqrt{x_{1}^{2}+x_{2}^{2}}+\left[r\left(g_{\zeta}(x)\right)\right] \partial_{i}\left(\frac{\xi(x)}{|x|^{2}} \sqrt{x_{1}^{2}+x_{2}^{2}}\right)\right]
\end{aligned}
$$

$$
\partial_{i}\left[\mathcal{F}_{3}^{\prime}(\zeta) \xi\right](x)=\left[-h^{\prime \prime}\left(\mathcal{M}(\zeta) \rho_{0}(x)\right) \mathcal{M}(\zeta) \rho_{0}(x) \partial_{i} \rho_{0}(x)-h^{\prime}\left(\mathcal{M}(\zeta) \rho_{0}(x)\right) \partial_{i} \rho_{0}(x)\right] \mathcal{M}^{\prime}(\zeta) \xi
$$

Proof. The $\partial_{i}$ derivative of the first term in (5.19) can be computed in a way similar to Lemma 5.2 by using the cutoff function $\chi$. This gives the first line of (5.35). We rewrite the second line of (5.19) as

$$
\begin{aligned}
& \int_{B_{2}} \rho_{0}\left(g_{\zeta}^{-1}\left(y^{\prime}\right)\right) \frac{-\left(g_{\zeta}(x)-y^{\prime}\right)}{\left|g_{\zeta}(x)-y^{\prime}\right|^{3}} d y^{\prime} \cdot \xi(x) \frac{x}{|x|^{2}} \\
= & -\int_{B_{2}} \rho_{0}\left(g_{\zeta}^{-1}\left(y^{\prime}\right)\right) \nabla_{y^{\prime}}\left(\frac{1}{\left|g_{\zeta}(x)-y^{\prime}\right|}\right) d y^{\prime} \cdot \xi(x) \frac{x}{|x|^{2}} \\
= & \sum_{j} \int_{B_{2}}\left(\nabla \rho_{0}\left(g_{\zeta}^{-1}\left(y^{\prime}\right)\right) \partial_{j} g_{\zeta}^{-1}\left(y^{\prime}\right)\right) \frac{1}{\left|g_{\zeta}(x)-y^{\prime}\right|} d y^{\prime} \xi(x) \frac{x_{j}}{|x|^{2}}
\end{aligned}
$$

Now we take the $\partial_{i}$ derivative using the cutoff method of Lemma 5.2. The last two lines of (5.35) follow. The calculations of the other terms are straightforward.

Lemma 5.4. With $F(x, s)$ defined in (5.15), the mixed derivatives are

$$
\partial_{s} \partial_{i} F(x, s)=\partial_{i} \partial_{s} F(x, s)=\partial_{i}\left[\mathcal{F}^{\prime}(\zeta+s \xi, \kappa) \xi\right](x)
$$

for every $s$ in a neighborhood of 0 .

Proof. The proof of Lemma 5.2 actually shows

$$
\partial_{s} F(x, s)=\left[\mathcal{F}^{\prime}(\zeta+s \xi, \omega) \xi\right](x),
$$

from which the second equality of (5.39) follows immediately. The other equality is certainly true if $F$ is $C^{2}$, but such a regularity is not yet established. Instead, we directly compute

$$
\begin{aligned}
\partial_{i} F(x, s) & =\partial_{i} \mathcal{F}(\zeta+s \xi, \omega)(x) \\
& =\mathcal{M}(\zeta+s \xi) \partial_{i} \mathcal{F}_{1}(\zeta+s \xi)(x)+\partial_{i} \mathcal{F}_{2}(\zeta+s \xi, \omega)(x)+\partial_{i} \mathcal{F}_{3}(\zeta+s \xi)(x)
\end{aligned}
$$

Thus

$$
\begin{aligned}
\partial_{s} \partial_{i} F(x, s)= & {\left[\partial_{s} \mathcal{M}(\zeta+s \xi)\right] \partial_{i} \mathcal{F}_{1}(\zeta+s \xi)(x)+\mathcal{M}(\zeta+s \xi)\left[\partial_{s} \partial_{i} \mathcal{F}_{1}(\zeta+s \xi)(x)\right] } \\
& +\partial_{s} \partial_{i} \mathcal{F}_{2}(\zeta+s \xi, \omega)(x)+\partial_{s} \partial_{i} \mathcal{F}_{3}(\zeta+s \xi)(x)
\end{aligned}
$$

We now compute $\partial_{s} \partial_{i} \mathcal{F}_{1}(\zeta+s \xi)(x)$. By (5.8),

$$
\partial_{i} \mathcal{F}_{1}(\zeta+s \xi)(x)=\left(\int_{B_{2}} \rho_{0}\left(g_{\zeta+s \xi}^{-1}\left(y^{\prime}\right)\right) \frac{-\left(g_{\zeta+s \xi}(x)-y^{\prime}\right)}{\left|g_{\zeta+s \xi}(x)-y^{\prime}\right|^{3}} d y^{\prime}\right) \cdot \partial_{i} g_{\zeta+s \xi}(x)
$$


We again use the cutoff function $\chi$ defined in Lemma 5.2 and write for every $x$ and component $j$ :

$$
I(s)=\int_{B_{2}} \rho_{0}\left(g_{\zeta+s \xi}^{-1}\left(y^{\prime}\right)\right) \frac{-\left(g_{\zeta+s \xi}(x)-y^{\prime}\right)_{j}}{\left|g_{\zeta+s \xi}(x)-y^{\prime}\right|^{3}} d y^{\prime}
$$

and

$$
I_{\epsilon}(s)=\int_{B_{2}} \rho_{0}\left(g_{\zeta+s \xi}^{-1}\left(y^{\prime}\right)\right) \frac{-\left(g_{\zeta+s \xi}(x)-y^{\prime}\right)_{j}}{\left|g_{\zeta+s \xi}(x)-y^{\prime}\right|^{3}}\left[1-\chi\left(\frac{\left|g_{\zeta+s \xi}(x)-y^{\prime}\right|}{\epsilon}\right)\right] d y^{\prime} .
$$

For $s$ in suitable neighborhood of $0, I_{\epsilon}(s)$ converges uniformly to $I(s)$, whereas $I_{\epsilon}^{\prime}(s)=$

$$
\begin{aligned}
& \int_{B_{2}}\left(\nabla \rho_{0}\left(g_{\zeta+s \xi}^{-1}\left(y^{\prime}\right)\right) \partial_{s} g_{\zeta+s \xi}^{-1}\left(y^{\prime}\right)\right) \frac{-\left(g_{\zeta+s \xi}(x)-y^{\prime}\right)_{j}}{\left|g_{\zeta+s \xi}(x)-y^{\prime}\right|^{3}}\left[1-\chi\left(\frac{\left|g_{\zeta+s \xi}(x)-y^{\prime}\right|}{\epsilon}\right)\right] d y^{\prime} \\
& -\int_{B_{2}} \rho_{0}\left(g_{\zeta+s \xi}^{-1}\left(y^{\prime}\right)\right)\left(\partial_{j} \partial_{s} \frac{1}{\left|g_{\zeta+s \xi}(x)-y^{\prime}\right|}\right)\left[1-\chi\left(\frac{\left|g_{\zeta+s \xi}(x)-y^{\prime}\right|}{\epsilon}\right)\right] d y^{\prime}
\end{aligned}
$$

$$
-\int_{B_{2}} \rho_{0}\left(g_{\zeta+s \xi}^{-1}\left(y^{\prime}\right)\right)\left(\partial_{j} \frac{1}{\left|g_{\zeta+s \xi}(x)-y^{\prime}\right|}\right) \chi^{\prime}\left(\frac{\left|g_{\zeta+s \xi}(x)-y^{\prime}\right|}{\epsilon}\right) \frac{1}{\epsilon} \partial_{s}\left|g_{\zeta+s \xi}(x)-y^{\prime}\right| d y^{\prime} .
$$

After integration by parts, the second term in (5.46) equals

$$
\begin{aligned}
& \int_{B_{2}}\left(\nabla \rho_{0}\left(g_{\zeta+s \xi}^{-1}\left(y^{\prime}\right)\right) \partial_{j} g_{\zeta+s \xi}^{-1}\left(y^{\prime}\right)\right)\left(\partial_{s} \frac{1}{\left|g_{\zeta+s \xi}(x)-y^{\prime}\right|}\right)\left[1-\chi\left(\frac{\left|g_{\zeta+s \xi}(x)-y^{\prime}\right|}{\epsilon}\right)\right] d y^{\prime} \\
& +\int_{B_{2}} \rho_{0}\left(g_{\zeta+s \xi}^{-1}\left(y^{\prime}\right)\right)\left(\partial_{s} \frac{1}{\left|g_{\zeta+s \xi}(x)-y^{\prime}\right|}\right) \chi^{\prime}\left(\frac{\left|g_{\zeta+s \xi}(x)-y^{\prime}\right|}{\epsilon}\right) \frac{1}{\epsilon} \partial_{j}\left|g_{\zeta+s \xi}(x)-y^{\prime}\right| d y^{\prime} .
\end{aligned}
$$

Noticing that

$$
\left(\partial_{s} \frac{1}{\left|g_{\zeta+s \xi}(x)-y^{\prime}\right|}\right) \partial_{j}\left|g_{\zeta+s \xi}(x)-y^{\prime}\right|=\left(\partial_{j} \frac{1}{\left|g_{\zeta+s \xi}(x)-y^{\prime}\right|}\right) \partial_{s}\left|g_{\zeta+s \xi}(x)-y^{\prime}\right|,
$$

we see that the last term in (5.47) cancels the last term in (5.46). Therefore

$$
\begin{aligned}
& I_{\epsilon}^{\prime}(s) \\
= & \int_{B_{2}}\left(\nabla \rho_{0}\left(g_{\zeta+s \xi}^{-1}\left(y^{\prime}\right)\right) \partial_{s} g_{\zeta+s \xi}^{-1}\left(y^{\prime}\right)\right) \frac{-\left(g_{\zeta+s \xi}(x)-y^{\prime}\right)_{j}}{\left|g_{\zeta+s \xi}(x)-y^{\prime}\right|^{3}}\left[1-\chi\left(\frac{\left|g_{\zeta+s \xi}(x)-y^{\prime}\right|}{\epsilon}\right)\right] d y^{\prime} \\
& (5.49) \\
& +\int_{B_{2}}\left(\nabla \rho_{0}\left(g_{\zeta+s \xi}^{-1}\left(y^{\prime}\right)\right) \partial_{j} g_{\zeta+s \xi}^{-1}\left(y^{\prime}\right)\right)\left(\partial_{s} \frac{1}{\left|g_{\zeta+s \xi}(x)-y^{\prime}\right|}\right)\left[1-\chi\left(\frac{\left|g_{\zeta+s \xi}(x)-y^{\prime}\right|}{\epsilon}\right)\right] d y^{\prime} .
\end{aligned}
$$

By (5.49) $I_{\epsilon}^{\prime}(s)$ converges uniformly to

$$
\begin{aligned}
& \int_{B_{2}}\left(\nabla \rho_{0}\left(g_{\zeta+s \xi}^{-1}\left(y^{\prime}\right)\right) \partial_{s} g_{\zeta+s \xi}^{-1}\left(y^{\prime}\right)\right) \frac{-\left(g_{\zeta+s \xi}(x)-y^{\prime}\right)_{j}}{\left|g_{\zeta+s \xi}(x)-y^{\prime}\right|^{3}} d y^{\prime} \\
& +\int_{B_{2}}\left(\nabla \rho_{0}\left(g_{\zeta+s \xi}^{-1}\left(y^{\prime}\right)\right) \partial_{j} g_{\zeta+s \xi}^{-1}\left(y^{\prime}\right)\right)\left(\partial_{s} \frac{1}{\left|g_{\zeta+s \xi}(x)-y^{\prime}\right|}\right) d y^{\prime}
\end{aligned}
$$


as $\epsilon \rightarrow 0$. Therefore $I(s)$ is $C^{1}$ and $I^{\prime}(s)$ is given by (5.50). (5.43), (5.44), and (5.50) now give

$$
\begin{aligned}
& \partial_{s} \partial_{i} \mathcal{F}_{1}(\zeta+s \xi)(x) \\
& =\int_{B_{2}}\left(\nabla \rho_{0}\left(g_{\zeta+s \xi}^{-1}\left(y^{\prime}\right)\right) \partial_{s} g_{\zeta+s \xi}^{-1}\left(y^{\prime}\right)\right) \frac{-\left(g_{\zeta+s \xi}(x)-y^{\prime}\right)}{\left|g_{\zeta+s \xi}(x)-y^{\prime}\right|^{3}} d y^{\prime} \cdot \partial_{i} g_{\zeta+s \xi} \\
& +\sum_{j} \int_{B_{2}}\left(\nabla \rho_{0}\left(g_{\zeta+s \xi}^{-1}\left(y^{\prime}\right)\right) \partial_{j} g_{\zeta+s \xi}^{-1}\left(y^{\prime}\right)\right)\left(\frac{-\left(g_{\zeta+s \xi}(x)-y^{\prime}\right)}{\left|g_{\zeta+s \xi}(x)-y^{\prime}\right|^{3}} \cdot \partial_{i} g_{\zeta+s \xi}(x)\right) d y^{\prime} \xi(x) \frac{x_{j}}{|x|^{2}} \\
& +\left(\int_{B_{2}} \rho_{0}\left(g_{\zeta+s \xi}^{-1}\left(y^{\prime}\right)\right) \frac{-\left(g_{\zeta+s \xi}(x)-y^{\prime}\right)}{\left|g_{\zeta+s \xi}(x)-y^{\prime}\right|^{3}} d y^{\prime}\right) \cdot \partial_{i}\left(\xi(x) \frac{x}{|x|^{2}}\right) \\
& =\partial_{i}\left[\mathcal{F}_{1}^{\prime}(\zeta+s \xi) \xi\right](x) .
\end{aligned}
$$

The calculations related to the other terms are straightforward and therefore omitted.

Next we show that the formal derivative is indeed a bounded linear map on $X$.

Lemma 5.5. If $\zeta \in B_{\epsilon}(X)$ and $\epsilon$ is small enough, there is a constant $C$ such that

$$
\left\|\mathcal{F}^{\prime}(\zeta, \kappa) \xi\right\|_{X} \leq C(1+\kappa)\|\xi\|_{X} .
$$

Proof. We estimate the terms in (5.34) one by one. First of all,

$$
\left|\mathcal{M}^{\prime}(\zeta) \xi\right| \leq C\left\|\rho_{0}\right\|_{1}\|\xi\|_{X}
$$

by (3.18) and (3.17). $\partial_{i}\left[\mathcal{F}_{1}(\zeta)\right](x)$ was already estimated in (5.9). Next we estimate $\partial_{i}\left[\mathcal{F}_{1}^{\prime}(\zeta) \xi\right](x)$ from (5.35). We call the three lines in (5.35) $I_{1}, I_{2}$ and $I_{3}$ respectively. We apply Lemma 3.6 to $I_{1}$ with

$$
f\left(y^{\prime}\right)=f_{1}\left(y^{\prime}\right)=\left(\nabla \rho_{0}\left(g_{\zeta}^{-1}\left(y^{\prime}\right)\right) D g_{\zeta}^{-1}\left(y^{\prime}\right) \frac{g_{\zeta}^{-1}\left(y^{\prime}\right)}{\left|g_{\zeta}^{-1}\left(y^{\prime}\right)\right|^{2}}\right) \xi\left(g_{\zeta}^{-1}\left(y^{\prime}\right)\right) .
$$

Note that $f_{1}$ is continuous and $\left|f_{1}\left(y^{\prime}\right)\right| \leq C\left\|\nabla \rho_{0}\right\|_{\infty}\|\xi\|_{X}\left|g_{\zeta}^{-1}\left(y^{\prime}\right)\right| \leq C\|\xi\|_{X}\left|y^{\prime}\right|$. Thus $\left|I_{1}\right| \leq$ $C\|\xi\|_{X}|x|$ for all $x \in \overline{B_{1}}$. Next we estimate the integral in $I_{2}$ directly as

$$
\begin{aligned}
& \left|\int_{B_{2}}\left(\nabla \rho_{0}\left(g_{\zeta}^{-1}\left(y^{\prime}\right)\right) \partial_{j} g_{\zeta}^{-1}\left(y^{\prime}\right)\right)\left(\frac{-\left(g_{\zeta}(x)-y^{\prime}\right)}{\left|g_{\zeta}(x)-y^{\prime}\right|^{3}} \cdot \partial_{i} g_{\zeta}(x)\right) d y^{\prime}\right| \\
\leq & C\left\|\nabla \rho_{0}\right\|_{\infty} \int_{B_{2}} \frac{1}{\left|g_{\zeta}(x)-y^{\prime}\right|^{2}} d y^{\prime} \leq C\left\|\nabla \rho_{0}\right\|_{\infty} \int_{B_{4}} \frac{1}{\left|y^{\prime}\right|^{2}} d y^{\prime} \leq C_{1}
\end{aligned}
$$

if $x \in \overline{B_{1}}$. On the other hand, $\frac{\left|\xi(x) x_{j}\right|}{|x|^{2}} \leq \frac{|\xi(x)|}{|x|} \leq C\|\xi\|_{X}|x|$ so that $\left|I_{2}\right| \leq C\|\xi\|_{X}|x|$. Next we apply Lemma 3.6 to $I_{3}$ with $f\left(y^{\prime}\right)=f_{3}\left(y^{\prime}\right)=\rho_{0}\left(g_{\zeta}^{-1}\left(y^{\prime}\right)\right)$. Note that $\left|f_{3}\left(y^{\prime}\right)-f_{3}(0)\right| \leq$ $\left\|\nabla \rho_{0}\right\|_{\infty}\left|g_{\zeta}^{-1}\left(y^{\prime}\right)\right| \leq C\left|y^{\prime}\right|$. Therefore

$$
\left|\int_{B_{2}} \rho_{0}\left(g_{\zeta}^{-1}\left(y^{\prime}\right)\right) \frac{-\left(g_{\zeta}(x)-y^{\prime}\right)}{\left|g_{\zeta}(x)-y^{\prime}\right|^{3}} d y^{\prime}\right| \leq C|x|
$$

for all $x \in \overline{B_{1}}$. Together with the fact that $\left|\partial_{i}\left(\xi(x) \frac{x}{|x|^{2}}\right)\right| \leq C\|\xi\|_{X}$, this implies $\left|I_{3}\right| \leq$ $C\|\xi\|_{X}|x|$. Combining the estimates on $I_{1}, I_{2}$ and $I_{3}$, we get

$$
\left|\partial_{i}\left[\mathcal{F}_{1}^{\prime}(\zeta) \xi\right](x)\right| \leq C\|\xi\|_{X}|x| .
$$

We next estimate $\partial_{i}\left[\mathcal{F}_{2}^{\prime}(\zeta) \xi\right](x)$ from (5.36). Due to (5.11) and (5.12), the first line of (5.36) is bounded by $C\|\xi\|_{X}|x|$ since $\left(\omega^{2}\right)^{\prime}$ is locally bounded. The second and third lines are bounded by $C\|\xi\|_{X}|x|$ since $\omega^{2}$ is locally bounded. $\partial_{i}\left[\mathcal{F}_{3}^{\prime}(\zeta, \kappa) \xi\right](x)$ is given in (5.37). It can be rewritten as

$$
-\left(\mathcal{M}^{\prime}(\zeta) \xi\right)\left[\mathcal{M} \frac{h^{\prime \prime}\left(\mathcal{M} \rho_{0}\right) \rho_{0}}{h^{\prime}\left(\rho_{0}\right)}+\frac{h^{\prime}\left(\mathcal{M} \rho_{0}\right)}{h^{\prime}\left(\rho_{0}\right)}\right] \partial_{i} u_{0} .
$$


By (3.3),

$$
\lim _{s \rightarrow 0^{+}} \frac{h^{\prime \prime}(\mathcal{M} s) s}{h^{\prime}(s)}=(\gamma-2) \mathcal{M}^{\gamma-3} .
$$

(5.59) and (5.14) imply that (5.58) is continuous on $\overline{B_{1}}$. Again using the fact that $\partial_{i} u_{0}(0)=0$ we get

$$
\left|\partial_{i}\left[\mathcal{F}_{3}^{\prime}(\zeta, \kappa) \xi\right](x)\right| \leq C\left|\mathcal{M}^{\prime}(\zeta) \xi\right||x| \leq C\|\xi\|_{X}|x|
$$

This completes all the estimates.

The next proposition asserts that the formal derivative depends continuously on $\zeta$.

Lemma 5.6. Let $\alpha=\min \left\{\frac{2-\gamma}{\gamma-1}, \beta(\omega)\right\}$, where $\beta(\omega)$ is given in Assumption (2.7). If $\zeta_{1}, \zeta_{2} \in$ $B_{\epsilon}(X)$ and $\epsilon$ is small enough, there is a constant $C$ such that

$$
\left\|\left(\mathcal{F}^{\prime}\left(\zeta_{1}, \kappa\right)-\mathcal{F}^{\prime}\left(\zeta_{2}, \kappa\right)\right) \xi\right\|_{X} \leq C(1+\kappa)\left\|\zeta_{1}-\zeta_{2}\right\|_{X}^{\alpha}\|\xi\|_{X} .
$$

Proof.

$$
\begin{gathered}
\mathcal{F}^{\prime}\left(\zeta_{1}, \kappa\right)-\mathcal{F}\left(\zeta_{2}, \kappa\right)=I_{1}+I_{2}+I_{3}+I_{4}+I_{5}+I_{6}, \\
I_{1}=\left[\mathcal{M}^{\prime}\left(\zeta_{1}\right) \xi-\mathcal{M}^{\prime}\left(\zeta_{2}\right) \xi\right] \mathcal{F}_{1}\left(\zeta_{1}\right), \quad I_{2}=\left[\mathcal{M}^{\prime}\left(\zeta_{2}\right) \xi\right]\left(\mathcal{F}_{1}\left(\zeta_{1}\right)-\mathcal{F}_{1}\left(\zeta_{2}\right)\right), \\
I_{3}=\left(\mathcal{M}\left(\zeta_{1}\right)-\mathcal{M}\left(\zeta_{2}\right)\right) \mathcal{F}_{1}^{\prime}\left(\zeta_{1}\right) \xi, \quad I_{4}=\mathcal{M}\left(\zeta_{2}\right)\left[\left(\mathcal{F}_{1}^{\prime}\left(\zeta_{1}\right)-\mathcal{F}_{1}^{\prime}\left(\zeta_{2}\right)\right) \xi\right], \\
I_{5}=\kappa\left(\mathcal{F}_{2}^{\prime}\left(\zeta_{1}\right)-\mathcal{F}_{2}^{\prime}\left(\zeta_{2}\right)\right) \xi, \quad I_{6}=\left(\mathcal{F}_{3}^{\prime}\left(\zeta_{1}\right)-\mathcal{F}_{3}^{\prime}\left(\zeta_{2}\right)\right) \xi .
\end{gathered}
$$

We estimate the terms $I_{1}$ through $I_{6}$ one by one. Starting with $I_{1}$, the $X$ norm of $\mathcal{F}_{1}\left(\zeta_{1}\right)$ was already estimated in Lemma 5.1. We just need to estimate the size of $\mathcal{M}^{\prime}\left(\zeta_{1}\right) \xi-\mathcal{M}^{\prime}\left(\zeta_{2}\right) \xi$. In (5.18) the function $\zeta$ appears in three different places. Here and in the following discussion we take the difference of the occurrences of $\zeta$ one at a time. For $\mathcal{M}^{\prime}(\zeta) \xi$ the key differences to estimate are $\operatorname{det} D g_{\zeta_{1}}(x)-\operatorname{det} D g_{\zeta_{2}}(x), D g_{\zeta_{1}}^{-1}(x)-D g_{\zeta_{2}}^{-1}(x)$ and

$$
\left(\int_{B_{1}} \rho_{0}(x) \operatorname{det} D g_{\zeta_{1}}(x) d x\right)^{-2}-\left(\int_{B_{1}} \rho_{0}(x) \operatorname{det} D g_{\zeta_{2}}(x) d x\right)^{-2} .
$$

We observe that all three differences are bounded by $C\left\|\zeta_{1}-\zeta_{2}\right\|_{X}$, either by directly using (3.34), (3.35), or by combining them with a simple application of the mean value theorem (in the case of (5.62)). Thus

$$
\left\|I_{1}\right\|_{X} \leq C\left\|\zeta_{1}-\zeta_{2}\right\|_{X}\|\xi\|_{X}
$$

We now turn our attention to $I_{2}$. Again $\mathcal{M}^{\prime}\left(\zeta_{2}\right) \xi$ was already estimated in Lemma 5.5 and satisfies $\left\|\mathcal{M}^{\prime}\left(\zeta_{2}\right) \xi\right\|_{X} \leq C\|\xi\|_{X}$. In order to estimate the $X$ norm of $\mathcal{F}_{1}\left(\zeta_{1}\right)-\mathcal{F}_{1}\left(\zeta_{2}\right)$, we use (5.8), which we break up into the three parts

$$
\begin{gathered}
I_{21}=\left(\int_{B_{2}}\left(\rho_{0}\left(g_{\zeta_{1}}^{-1}\left(y^{\prime}\right)\right)-\rho_{0}\left(g_{\zeta_{2}}^{-1}\left(y^{\prime}\right)\right)\right) \frac{-\left(g_{\zeta_{j}}(x)-y^{\prime}\right)}{\left|g_{\zeta_{j}}(x)-y^{\prime}\right|^{3}} d y^{\prime}\right) \cdot \partial_{i} g_{\zeta_{k}}(x), \\
I_{22}=\left(\int_{B_{2}} \rho_{0}\left(g_{\zeta_{j}}^{-1}\left(y^{\prime}\right)\right)\left(\frac{-\left(g_{\zeta_{1}}(x)-y^{\prime}\right)}{\left|g_{\zeta_{1}}(x)-y^{\prime}\right|^{3}}-\frac{-\left(g_{\zeta_{2}}(x)-y^{\prime}\right)}{\left|g_{\zeta_{2}}(x)-y^{\prime}\right|^{3}}\right) d y^{\prime}\right) \cdot \partial_{i} g_{\zeta_{k}}(x)
\end{gathered}
$$

and

$$
I_{23}=\left(\int_{B_{2}} \rho_{0}\left(g_{\zeta_{j}}^{-1}\left(y^{\prime}\right)\right) \frac{-\left(g_{\zeta_{k}}(x)-y^{\prime}\right)}{\left|g_{\zeta_{k}}(x)-y^{\prime}\right|^{3}} d y^{\prime}\right) \cdot \partial_{i}\left(g_{\zeta_{1}}(x)-g_{\zeta_{2}}(x)\right) .
$$

Here $j, k$ can be 1 or 2 . To estimate $I_{21}$, we apply Lemma 3.6 to $f\left(y^{\prime}\right)=f_{1}\left(y^{\prime}\right)=$ $\rho_{0}\left(g_{\zeta_{1}}^{-1}\left(y^{\prime}\right)\right)-\rho_{0}\left(g_{\zeta_{2}}^{-1}\left(y^{\prime}\right)\right)$. Note that $f_{1}$ is continuous, $f_{1}(0)=0$ and

$$
\left|f_{1}(x)\right| \leq\left\|\nabla \rho_{0}\right\|_{\infty}\left|g_{\zeta_{1}}^{-1}\left(y^{\prime}\right)-g_{\zeta_{2}}^{-1}\left(y^{\prime}\right)\right| \leq C\left\|\zeta_{1}-\zeta_{2}\right\|_{X}\left|y^{\prime}\right|
$$

by (3.37). Therefore $\left|I_{21}\right| \leq C\left\|\zeta_{1}-\zeta_{2}\right\|_{X}|x|$. To estimate $I_{22}$ we apply Lemma 3.6 to $f\left(y^{\prime}\right)=$ $f_{2}\left(y^{\prime}\right)=\rho_{0}\left(g_{\zeta_{j}}^{-1}\left(y^{\prime}\right)\right)$, which is a continuous function that satisfies

$$
\left|f_{2}\left(y^{\prime}\right)-f_{2}(0)\right| \leq\left\|\nabla \rho_{0}\right\|_{\infty}\left|g_{\zeta_{j}}^{-1}\left(y^{\prime}\right)\right| \leq C\left|y^{\prime}\right| .
$$


Therefore $\left|I_{22}\right| \leq C\left\|\zeta_{1}-\zeta_{2}\right\|_{X}^{\beta}|x|$. Finally, $\left|I_{23}\right| \leq C\left\|\zeta_{1}-\zeta_{2}\right\|_{X}|x|$ by a similar application of Lemma 3.6 and (3.36). Thus $\left\|I_{2}\right\|_{X} \leq C\left\|\zeta_{1}-\zeta_{2}\right\|_{X}^{\beta}\|\xi\|_{X}$. The estimation of $I_{3}$ is similar to that of $I_{1}$ and is omitted.

We focus our attention now on $I_{4}=\mathcal{M}\left(\zeta_{2}\right)\left[\left(\mathcal{F}_{1}^{\prime}\left(\zeta_{1}\right)-\mathcal{F}_{1}^{\prime}\left(\zeta_{2}\right)\right) \xi\right]$. We need to estimate $\left\|\left(\mathcal{F}_{1}^{\prime}\left(\zeta_{1}\right)-\mathcal{F}_{1}^{\prime}\left(\zeta_{2}\right)\right) \xi\right\|_{X}$ starting from (5.35). This is a long equation with three lines, hence $\partial_{i}\left[\left(\mathcal{F}_{1}^{\prime}\left(\zeta_{1}\right)-\mathcal{F}_{1}^{\prime}\left(\zeta_{2}\right)\right) \xi\right](x)$ can also be broken into three lines, which we denote by $I_{41}, I_{42}$ and $I_{43}$ respectively. For the first line we must estimate

$$
\begin{gathered}
I_{41 a}=\int_{B_{2}}\left(\mathcal{G}\left(\zeta_{1}\right)-\mathcal{G}\left(\zeta_{2}\right)\right) \frac{-\left(g_{\zeta_{j}}(x)-y^{\prime}\right)}{\left|g_{\zeta_{j}}(x)-y^{\prime}\right|^{3}} d y^{\prime} \cdot \partial_{i} g_{\zeta_{k}}(x), \\
I_{41 b}=\int_{B_{2}} \mathcal{G}\left(\zeta_{j}\right)\left(\frac{-\left(g_{\zeta_{1}}(x)-y^{\prime}\right)}{\left|g_{\zeta_{1}}(x)-y^{\prime}\right|^{3}}-\frac{-\left(g_{\zeta_{2}}(x)-y^{\prime}\right)}{\left|g_{\zeta_{2}}(x)-y^{\prime}\right|^{3}}\right) d y^{\prime} \cdot \partial_{i} g_{\zeta_{k}}(x), \\
I_{41 c}=\int_{B_{2}} \mathcal{G}\left(\zeta_{j}\right) \frac{-\left(g_{\zeta_{k}}(x)-y^{\prime}\right)}{\left|g_{\zeta_{k}}(x)-y^{\prime}\right|^{3}} d y^{\prime} \cdot \partial_{i}\left(g_{\zeta_{1}}(x)-g_{\zeta_{2}}(x)\right),
\end{gathered}
$$

$(j, k=1,2)$, where for brevity we denote

$$
\mathcal{G}(\zeta)=\nabla \rho_{0}\left(g_{\zeta}^{-1}\left(y^{\prime}\right)\right) D g_{\zeta}^{-1}\left(y^{\prime}\right) \xi\left(g_{\zeta}^{-1}\left(y^{\prime}\right)\right) \frac{g_{\zeta}^{-1}\left(y^{\prime}\right)}{\left|g_{\zeta}^{-1}\left(y^{\prime}\right)\right|^{2}}
$$

We again apply Lemma 3.6 to these integrals. For $I_{41 a}$, the function $f\left(y^{\prime}\right)=\mathcal{G}\left(\zeta_{1}\right)-\mathcal{G}\left(\zeta_{2}\right)$ is continuous and satisfies

$$
\begin{aligned}
& \quad\left|f\left(y^{\prime}\right)\right| \leq C\left\|\rho_{0}\right\|_{C^{1, \alpha}}\left|g_{\zeta_{1}}^{-1}\left(y^{\prime}\right)-g_{\zeta_{2}}^{-1}\left(y^{\prime}\right)\right|^{\alpha}\|\xi\|_{X}\left|y^{\prime}\right| \\
& \quad+C\left\|\nabla \rho_{0}\right\|_{\infty}\left|D g_{\zeta_{1}}^{-1}\left(y^{\prime}\right)-D g_{\zeta_{2}}^{-1}\left(y^{\prime}\right)\right|\|\xi\|_{X}\left|y^{\prime}\right|+C\left\|\nabla \rho_{0}\right\|_{\infty}\|\nabla \xi\|_{\infty}\left|g_{\zeta_{1}}^{-1}\left(y^{\prime}\right)-g_{\zeta_{2}}^{-1}\left(y^{\prime}\right)\right| \frac{1}{\left|g_{\zeta_{j}}\left(y^{\prime}\right)\right|} \\
& \quad+C\left\|\nabla \rho_{0}\right\|_{\infty}\|\xi\|_{X}\left|y^{\prime}\right|^{2} \frac{1}{\left|\theta g_{\zeta_{1}}^{-1}\left(y^{\prime}\right)+(1-\theta) g_{\zeta_{2}}^{-1}\left(y^{\prime}\right)\right|^{2}}\left|g_{\zeta_{1}}^{-1}\left(y^{\prime}\right)-g_{\zeta_{2}}^{-1}\left(y^{\prime}\right)\right| \\
& \leq C\left\|\rho_{0}\right\|_{C^{1, \alpha}}\left\|\zeta_{1}-\zeta_{2}\right\|_{X}^{\alpha}\|\xi\|_{X}\left|y^{\prime}\right| .
\end{aligned}
$$

Lemma 3.6 now implies $\left|I_{41 a}\right| \leq C\left\|\zeta_{1}-\zeta_{2}\right\|_{X}^{\alpha}\|\xi\|_{X}|x|$. The terms $I_{41 b}$ and $I_{41 c}$ are estimated similarly. As for $I_{42}$, we write

$$
\begin{gathered}
I_{42 a}=\int_{B_{2}}\left(\mathcal{G}\left(\zeta_{1}\right)-\mathcal{G}\left(\zeta_{2}\right)\right)\left(\frac{-\left(g_{\zeta_{k}}(x)-y^{\prime}\right)}{\left|g_{\zeta_{k}}(x)-y^{\prime}\right|^{3}} \cdot \partial_{i} g_{\zeta_{l}}(x)\right) d y^{\prime}, \\
I_{42 b}=\int_{B_{2}} \mathcal{G}\left(\zeta_{k}\right)\left(\frac{-\left(g_{\zeta_{1}}(x)-y^{\prime}\right)}{\left|g_{\zeta_{1}}(x)-y^{\prime}\right|^{3}}-\frac{-\left(g_{\zeta_{2}}(x)-y^{\prime}\right)}{\left|g_{\zeta_{2}}(x)-y^{\prime}\right|^{3}}\right) \cdot \partial_{i} g_{\zeta_{l}}(x) d y^{\prime}, \\
I_{42 c}=\int_{B_{2}} \mathcal{G}\left(\zeta_{k}\right) \frac{-\left(g_{\zeta_{l}}(x)-y^{\prime}\right)}{\left|g_{\zeta_{l}}(x)-y^{\prime}\right|^{3}} \cdot\left(\partial_{i} g_{\zeta_{1}}(x)-\partial_{i} g_{\zeta_{2}}(x)\right) d y
\end{gathered}
$$

where now $\mathcal{G}(\zeta)=\nabla \rho_{0}\left(g_{\zeta}^{-1}\left(y^{\prime}\right)\right) \partial_{j} g_{\zeta}^{-1}\left(y^{\prime}\right)$. These terms are estimated in a similar fashion, as are the estimates on $I_{43}$. Thus we get

$$
\left\|I_{4}\right\|_{X} \leq C\left\|\zeta_{1}-\zeta_{2}\right\|_{X}^{\alpha}\|\xi\|_{X}
$$

To estimate $I_{5}$, we use (5.36). We only need

$$
\left(\omega^{2}\right)^{\prime}\left[r\left(g_{\zeta_{1}}(x)\right)\right]-\left(\omega^{2}\right)^{\prime}\left[r\left(g_{\zeta_{2}}(x)\right)\right] \quad \text { and } \quad\left(\omega^{2}\right)\left[r\left(g_{\zeta_{1}}(x)\right)\right]-\left(\omega^{2}\right)\left[r\left(g_{\zeta_{2}}(x)\right)\right]
$$

to be bounded by $C\left\|\zeta_{1}-\zeta_{2}\right\|_{X}^{\beta}$, which is true since $\omega^{2}$ is locally $C^{1, \beta}$ on $[0, \infty)$. Thus

$$
\left|\partial_{i}\left[\mathcal{F}_{2}^{\prime}\left(\zeta_{1}\right) \xi-\mathcal{F}_{2}^{\prime}\left(\zeta_{2}\right) \xi\right](x)\right| \leq C\left\|\zeta_{1}-\zeta_{2}\right\|_{X}^{\alpha}\|\xi\|_{X}|x|
$$

so that $\left\|I_{5}\right\|_{X} \leq C \kappa\left\|\zeta_{1}-\zeta_{2}\right\|_{X}^{\alpha}\|\xi\|_{X}$. 
Finally to estimate $I_{6}$, we calculate $\partial_{i}\left[\left(\mathcal{F}_{3}^{\prime}\left(\zeta_{1}\right)-\mathcal{F}_{3}^{\prime}\left(\zeta_{2}\right)\right) \xi\right]$ using (5.58) and splitting it into several pieces each involving a single difference as before. For instance, one piece is

$$
I_{63}=\left[\left(\mathcal{M}_{j}^{\prime}\right) \xi\right]\left[\mathcal{M}_{k} \frac{\left[h^{\prime \prime}\left(\mathcal{M}_{1} \rho_{0}\right)-h^{\prime \prime}\left(\mathcal{M}_{2} \rho_{0}\right)\right] \rho_{0}}{h^{\prime}\left(\rho_{0}\right)}\right] \partial_{i} u_{0},
$$

where we use the shorthand $\mathcal{M}_{j}=\mathcal{M}\left(\zeta_{j}\right)$ with $j, k, l=1,2$. We write

$$
\frac{\left[h^{\prime \prime}\left(\mathcal{M}_{1} \rho_{0}\right)-h^{\prime \prime}\left(\mathcal{M}_{2} \rho_{0}\right)\right] \rho_{0}}{h^{\prime}\left(\rho_{0}\right)}=\left(M_{1}-M_{2}\right) \frac{h^{\prime \prime \prime}\left(\overline{\mathcal{M}} \rho_{0}\right) \rho_{0}^{2}}{h^{\prime}\left(\rho_{0}\right)}
$$

where $\overline{\mathcal{M}}$ is between $\mathcal{M}_{1}$ and $\mathcal{M}_{2}$. By $(3.3), \frac{h^{\prime \prime \prime}(\overline{\mathcal{M}} s) s^{2}}{h^{\prime}(s)}$ is bounded if $\overline{\mathcal{M}}$ is close to 1 and $s$ is close to zero. So the expression in (5.79) is bounded by $C\left|\mathcal{M}_{1}-\mathcal{M}_{2}\right| \leq C\left\|\zeta_{1}-\zeta_{2}\right\|_{X}$ and we get $\left|I_{63}\right| \leq C\left\|\zeta_{1}-\zeta_{2}\right\|_{X}\|\xi\|_{X}|x|$. Therefore $\left\|I_{6}\right\|_{X} \leq C\left\|\zeta_{1}-\zeta_{2}\right\|_{X}\|\xi\|_{X}$. This completes all the estimates needed to establish (5.61).

We are finally ready to show that the formal derivative is a genuine Fréchet derivative.

Lemma 5.7. Let $\zeta \in B_{\epsilon}(X)$, and $\xi$ be such that $\zeta+s \xi \in B_{\epsilon}(X)$ for all $s \in[-1,1]$. Then

$$
\left\|\mathcal{F}(\zeta+\xi, \kappa)-\mathcal{F}(\zeta, \kappa)-\mathcal{F}^{\prime}(\zeta, \kappa) \xi\right\|_{X} \leq C(1+\kappa)\|\xi\|_{X}^{1+\alpha},
$$

where $\mathcal{F}^{\prime}(\zeta, \kappa) \xi$ denotes the formal derivative defined in (5.17).

Proof. To estimate the $X$ norm in (5.80), we write

$$
\begin{aligned}
& \partial_{i}(\mathcal{F}(\zeta+\xi, \kappa)-\mathcal{F}(\zeta, \kappa))(x)=\partial_{i} F(x, 1)-\partial_{i} F(x, 0) \\
& =\partial_{s} \partial_{i} F(x, \theta)=\partial_{i}\left[\mathcal{F}^{\prime}(\zeta+\theta(x) \xi, \kappa) \xi\right](x)
\end{aligned}
$$

for some $\theta(x) \in(0,1)$ by Lemma [5.4. Thus

$$
\begin{aligned}
& \left|\partial_{i}(\mathcal{F}(\zeta+\xi, \kappa)-\mathcal{F}(\zeta, \kappa))(x)-\partial_{i}\left[\mathcal{F}^{\prime}(\zeta, \kappa) \xi\right](x)\right| \\
= & \left.\left|\partial_{i}\left[\mathcal{F}^{\prime}(\zeta+s \xi, \kappa) \xi\right](x)-\partial_{i}\left[\mathcal{F}^{\prime}(\zeta, \kappa) \xi\right](x)\right|\right|_{s=\theta(x)} \leq C(1+\kappa)\|\xi\|_{X}^{1+\alpha}|x|
\end{aligned}
$$

by Lemma 5.6, as desired.

Lemma 5.7 means that $\zeta \mapsto \mathcal{F}(\zeta, \kappa)$ is $C^{1}$ Fréchet differentiable with Fréchet derivative given by $\mathcal{F}^{\prime}(\zeta, \kappa)$. In fact, $\mathcal{F}$ is jointly Fréchet differentiable in both variables as the derivative with respect to $\kappa$ is very simple.

Lemma 5.8. $\mathcal{F}: B_{\epsilon}(X) \times \mathbb{R} \rightarrow X$ is continuously Fréchet differentiable with Fréchet derivative at $(\zeta, \kappa)$ given by

$$
(\xi, v) \mapsto \mathcal{F}^{\prime}(\zeta, \kappa) \xi+v \mathcal{F}_{2}(\zeta)
$$

Proof. By Lemma 5.6 and Lemma 5.7.

$$
\begin{aligned}
& \left\|\mathcal{F}(\zeta+\xi, \kappa+v)-\mathcal{F}(\zeta, \kappa)-\mathcal{F}^{\prime}(\zeta, \kappa) \xi-v \mathcal{F}_{2}(\zeta)\right\|_{X} \\
\leq & \left\|\mathcal{F}(\zeta+\xi, \kappa+v)-\mathcal{F}(\zeta+\xi, \kappa)-v \mathcal{F}_{2}(\zeta)\right\|_{X}+\left\|\mathcal{F}(\zeta+\xi, \kappa)-\mathcal{F}(\zeta, \kappa)-\mathcal{F}^{\prime}(\zeta, \kappa) \xi\right\|_{X} \\
\leq & |v|\left\|\mathcal{F}_{2}(\zeta+\xi)-\mathcal{F}_{2}(\zeta)\right\|_{X}+\left\|\mathcal{F}(\zeta+\xi, \kappa)-\mathcal{F}(\zeta, \kappa)-\mathcal{F}^{\prime}(\zeta, \kappa) \xi\right\|_{X} \\
\leq & C|v|\|\xi\|_{X}+C(1+\kappa)\|\xi\|_{X}^{1+\alpha}
\end{aligned}
$$

if $\|\xi\|_{X}$ and $|v|$ are small enough, and $\zeta \in B_{\epsilon}(X)$. Hence $\mathcal{F}$ is Fréchet differentiable with Fréchet derivative given in (5.83). The continuity of the mapping in (5.83) is easily obtained in a similar fashion using Lemma 5.6.

This completes the proof of Theorem 5.1 . 


\section{VLASOV MODEL}

6.1. Main theorem. The Vlasov-Poisson system (VP) is

$$
\begin{gathered}
\partial_{t} f+v \cdot \nabla_{x} f+\nabla_{x} U \cdot \nabla_{v} f=0, \quad f \geq 0, \\
-\Delta U=4 \pi \rho, \quad \rho=\int_{\mathbb{R}^{3}} f d v
\end{gathered}
$$

for $x, v \in \mathbb{R}^{3}$. Any function of the energy $\frac{1}{2} v^{2}-U$ and the angular momentum $x \times v$ automatically satisfies the steady Vlasov equation (6.1) with $\partial_{t} f=0$. Therefore we specialize to the following form for the microscopic density $f(x, v)$. Let

$$
f(x, v)=\frac{M}{D(\kappa, U)} \phi\left(\frac{1}{2} v^{2}-U(x), \kappa\left(x_{1} v_{2}-x_{2} v_{1}\right)\right), \quad x, v \in \mathbb{R}^{3},
$$

where

$$
D(\kappa, U)=\iint_{\mathbb{R}^{6}} \phi\left(\frac{1}{2} v^{2}-U(x), \kappa\left(x_{1} v_{2}-x_{2} v_{1}\right)\right) d v d x
$$

where we denote $v^{2}=|v|^{2}$ for brevity. Division by the integral $D(\kappa, U)$ assures us that the mass $\iint_{\mathbb{R}^{6}} f d v d x=M$ is a constant independent of the perturbation. The constant parameter $\kappa$ quantifies the smallness of the rotation. If $\kappa=0$, there is no rotation. Such an $f$ automatically satisfies (6.1). Of course, the potential $U$ must still be chosen to satisfy the Poisson equation (6.2).

The following assumptions, which imply that the integrals are finite, are made on $\phi$.

$$
\begin{aligned}
& \phi(E, L)> 0 \text { for } E<0, \quad \phi(E, L)=0 \text { for } E>0, \\
& \phi \in C^{1}((-\infty, 0) \times(-\infty, \infty)) \text { and } \partial_{L}^{2} \phi \in C((-\infty, 0) \times(-\infty, \infty)), \\
& \lim _{E \rightarrow 0^{-}}(-E)^{\mu} \phi(E, L)= \lim _{E \rightarrow 0^{-}}(-E)^{1 / 2} \partial_{L}^{2} \phi(E, L)=0 \quad \text { for some } 0<\mu<1 / 2, \\
& \lim _{E \rightarrow-\infty}(-E)^{1 / 2} \phi(E, L)=\infty, \\
& \lim _{E \rightarrow-\infty}(-E)^{-7 / 2} \phi(E, L)=0, \\
& \partial_{L} \phi(E, 0)=0 \text { for } E<0 .
\end{aligned}
$$

Each of the limits is assumed to be uniform for bounded $L$. We remark that the only place that $\mu \neq 1 / 2$ is needed is in the proof of (8.6) below. As mentioned in the introduction, our prime example is $\phi(E, L)=(\max (-E, 0))^{-\alpha} \psi(L)$, where $-\frac{7}{2}<\alpha<\frac{1}{2}$ and $\psi$ is any function such that $\psi^{\prime}(0)=0$.

The existence of radial (spherically symmetric) solutions with $\kappa=0$, as we now state, is well-known in the kinetic literature. By a radial solution we mean that $\kappa=0$ and $U$ is a function of $|x|$ only.

Proposition 6.1. Let $\phi$ satisfy the assumptions above. Given $R>0$, there exists a solution $\left(f_{0}, U_{0}\right)$ of (6.2), (6.3) with $\kappa=0$ that is radial, for which

- $U_{0}>0$ in $B_{R}, U_{0}=0$ on $\partial B_{R}, U_{0}<0$ in $\mathbb{R}^{3} \backslash B_{R}$,

- $U_{0}^{\prime}(|x|)<0$ for all $|x|>0$,

- $\rho_{0}>0$ in $B_{R}, \rho_{0}=0$ in $\mathbb{R}^{3} \backslash B_{R}$,

- $\rho_{0} \in C^{1, \nu}\left(\mathbb{R}^{3}\right)$ and $U_{0} \in C^{3, \nu}\left(\mathbb{R}^{3}\right)$ where $\nu=\frac{1}{2}-\mu$ and $\mu$ is given in (6.7).

We shall prove this proposition later in this section. The mass of the radial solution is defined as $M=\int_{\mathbb{R}^{3}} \rho_{0} d x=\iint_{\mathbb{R}^{6}} f_{0} d v d x=D\left(0, U_{0}\right)$ by (6.3). This is the constant $M$ that appears in (6.3). Our main theorem regarding non-radial solutions of the Vlasov model is as follows. 
Theorem 6.1. There exists $\bar{\kappa}>0$ such that for all $|\kappa|<\bar{\kappa}$ there exists a solution $\left(f_{\kappa}, U_{\kappa}\right)$ of VP of the form (6.3) with $f_{\kappa}$ axisymmetric and even in $x_{3}, U_{\kappa} \in C^{3}\left(\mathbb{R}^{3}\right)$,

$$
\iint_{\mathbb{R}^{6}} f_{\kappa}(x, v) d v d x=\int_{\mathbb{R}^{3}} \rho_{\kappa}(x) d x=M
$$

and the support of $\rho_{\kappa}$ is a compact set close to $\bar{B}_{1}$. The mapping $\kappa \rightarrow U_{\kappa}$ is continuous from $(-\bar{\kappa}, \bar{\kappa})$ to $C^{3}\left(\mathbb{R}^{3}\right)$.

6.2. Construction. As is explained above, with the given ansatz (6.3), the unknown is $U$ and the equation to be solved is the Poisson equation, $-\Delta U=4 \pi \rho$. A change of variables in (6.3) leads to the formula

$$
\rho(x)=\frac{M}{D(\kappa, U)} w(\kappa, r(x), U(x)),
$$

where

and

$$
D(\kappa, U)=\int_{\mathbb{R}^{3}} w(\kappa, r, U) d y,
$$

$$
\begin{aligned}
w(\kappa, r, u) & =\int_{\mathbb{R}^{3}} \phi\left(\frac{1}{2} v^{2}-u, \kappa\left(x_{1} v_{2}-x_{2} v_{1}\right)\right) d v \\
& =2 \pi \int_{-u}^{0} \int_{-\sqrt{2(E+u)}}^{\sqrt{2(E+u)}} \phi(E, \kappa r s) d s d E .
\end{aligned}
$$

Here $r=r(x)=\sqrt{x_{1}^{2}+x_{2}^{2}}$. Note that, due to (6.5), $w(\kappa, r, u)>0$ for $u>0$, while $w(\kappa, r, u)=0$ for $u<0$. Thus $w$ is supported essentially where $u$ is positive.

Indeed, we justify formula (6.12) as follows. Because we may choose coordinates $v_{1}=$ $\sigma \cos \theta, v_{2}=s, v_{3}=\sigma \sin \theta$ and $x_{2}=0, x_{1}=r$, the $v$-integral equals

$$
w(\kappa, r, u)=2 \pi \int_{\mathbb{R}} \int_{0}^{\infty} \phi\left(\frac{1}{2} s^{2}+\frac{1}{2} \sigma^{2}-u, \kappa r s\right) \sigma d \sigma d s,
$$

after which we introduce the variable $E=\frac{1}{2} s^{2}+\frac{1}{2} \sigma^{2}-u$ to obtain the double integral in (6.12).

With this notation the Poisson equation to be solved takes the form

$$
-\Delta U=\frac{4 \pi M}{D(\kappa, U)} w(\kappa, r, U) \quad \text { in } \mathbb{R}^{3},
$$

or equivalently,

$$
-U(z)+\frac{M}{D(\kappa, U)} \int_{\mathbb{R}^{3}} w(\kappa, r(y), U(y)) \frac{1}{|z-y|} d y=\text { constant }
$$

for $z \in \mathbb{R}^{3}$.

As with the Euler model, the solutions will be perturbations of the radial solution given in Proposition 6.1, as we shall now describe. As in Section 2 we can assume without loss of generality that $R=1$. We deform the domain exactly as in the Euler model, namely, by the homeomorphism

$$
g_{\zeta}(x)=\left(1+\frac{\zeta(x)}{|x|^{2}}\right) x, \quad x \in \overline{B_{1}},
$$

for some axisymmetric functions $\zeta: \overline{B_{1}} \rightarrow \mathbb{R}$ in $X$ (and extend it when necessary to $\mathbb{R}^{3}$ by Lemma 3.5). The Banach space $X$ is the same as in the Euler case, namely (2.20), (2.21). Our construction differs from that of [17] in three respects.

(i) the rescaling factor in (6.3) keeps the total mass unchanged,

(ii) the axisymmetric scaling has an $|x|^{2}$ factor on the denominator, and

(iii) the form of $\phi$ is generalized. 
The difference (i) is our main improvement of the basic physical construction, (ii) has certain technical advantages, and (iii) allows more general axisymmetric solutions.

We look for solutions to (6.13) of the form $U=U_{0}\left(g_{\zeta}^{-1}(z)\right)$. An important observation is that, we essentially only need (6.14) to hold for $z \in g_{\zeta}\left(B_{1}\right)$.

Lemma 6.1. Suppose $\zeta \in X$ with $\|\zeta\|_{X}$ small, and such that

$$
\begin{aligned}
& U_{0}\left(g_{\zeta}^{-1}(z)\right) \\
= & U_{0}(0)+\frac{M}{D\left(\kappa, U_{0} \circ g_{\zeta}^{-1}\right)} \int_{\mathbb{R}^{3}} w\left(\kappa, r(y), U_{0}\left(g_{\zeta}^{-1}(y)\right)\right)\left[\frac{1}{|z-y|}-\frac{1}{|y|}\right] d y
\end{aligned}
$$

for all $z \in g_{\zeta}\left(\overline{B_{1}}\right)$, then

$$
U(z)=U_{0}(0)+\frac{M}{D\left(\kappa, U_{0} \circ g_{\zeta}^{-1}\right)} \int_{\mathbb{R}^{3}} w\left(\kappa, r(y), U_{0}\left(g_{\zeta}^{-1}(y)\right)\right)\left[\frac{1}{|z-y|}-\frac{1}{|y|}\right] d y
$$

solves (6.13) for all $z \in \mathbb{R}^{3}$. Here the function $w\left(\kappa, r(y), U_{0}\left(g_{\zeta}^{-1}(y)\right)\right)$ is supported on $g_{\zeta}\left(\overline{B_{1}}\right)$.

Proof. We may think of $g_{\zeta}$ as being extended to $\mathbb{R}^{3}$, so that $U_{0} \circ g_{\zeta}^{-1}$ is a globally defined function. The definition of $U$ does not depend on how $g_{\zeta}$ is extended outside $B_{1}$, because $w\left(\kappa, r, U_{0} \circ g_{\zeta}^{-1}\right)$ is supported on $g_{\zeta}\left(\overline{B_{1}}\right)$. That in turn follows from Proposition 6.1 and the property $w(\kappa, r, u)=0$ if $u \leq 0$ mentioned above. By the regularity of $w$ established in Lemma 8.1 below, we have $U \in C^{2}\left(\mathbb{R}^{3}\right)$ with

$$
-\Delta U=\frac{4 \pi M}{D\left(\kappa, U_{0} \circ g_{\zeta}^{-1}\right)} w\left(\kappa, r, U_{0} \circ g_{\zeta}^{-1}\right)
$$

It remains to show that $w\left(\kappa, r, U_{0} \circ g_{\zeta}^{-1}\right)=w(\kappa, r, U)$. In fact, this is obviously true on $g_{\zeta}\left(\overline{B_{1}}\right)$, since $U=U_{0} \circ g_{\zeta}^{-1}$ there by definition. To show that $w\left(\kappa, r, U_{0} \circ g_{\zeta}^{-1}\right)$ and $w(\kappa, r, U)$ also agree on $\mathbb{R}^{3} \backslash g_{\zeta}\left(\overline{B_{1}}\right)$, we just need $U<0$ on $\mathbb{R}^{3} \backslash g_{\zeta}\left(\overline{B_{1}}\right)$. That this is true follows immediately from the facts that $U$ is harmonic on $\mathbb{R}^{3} \backslash g_{\zeta}\left(\overline{B_{1}}\right)$, that $U=U_{0} \circ g_{\zeta}^{-1}=0$ on $g_{\zeta}\left(\partial B_{1}\right)$, and that $U$ tends to a negative constant at infinity, by an application of the maximum principle.

We have reduced the problem to solving (6.16). Let us substitute $z=g_{\zeta}(x)$. Define the operator

$\mathcal{F}(\zeta, \kappa)(x)=-U_{0}(x)+U_{0}(0)+\frac{M}{D\left(\kappa, U_{0} \circ g_{\zeta}^{-1}\right)} \int_{B_{2}} w\left(\kappa, r(y), U_{0}\left(g_{\zeta}^{-1}(y)\right)\right)\left[\frac{1}{\left|g_{\zeta}(x)-y\right|}-\frac{1}{|y|}\right] d y$

for $x$ in the unit ball $B_{1}$. Thus our goal reduces to solving $\mathcal{F}(\zeta, \kappa)=0$ for the function $\zeta \in X$ as a function of the parameter $\kappa$ for small $\kappa$. We have chosen the constant terms conveniently so that $\mathcal{F}(0,0)=0$, which corresponds to the radial solution.

6.3. Radial solutions. With $\kappa=0$ we have, from (6.2) and (6.12),

$$
-\Delta U_{0}=4 \pi \rho_{0}=4 \pi w\left(0,0, U_{0}\right)=16 \pi^{2} \sqrt{2} \int_{-U_{0}(x)}^{0} \phi(E, 0) \sqrt{U_{0}(x)+E} d E .
$$

Defining

$$
G(u)=w(0,0, u)=4 \pi \sqrt{2} \int_{-u}^{0} \phi(E, 0) \sqrt{u+E} d E,
$$

we can rewrite 6.20 as

$$
-\Delta U_{0}=4 \pi G\left(U_{0}\right)
$$


Due to (6.5), $G(u)$ vanishes for $u<0$ and is positive for $u>0$. We can convert the conditions on $\phi$ given above to some conditions of $G$ as follows.

Lemma 6.2. If $\phi$ satisfies the conditions (6.5) - then $G \in C^{1, \nu}(\mathbb{R}), G(u)=0$ for $u \leq 0$, $G(u)>0$ for $u>0$, and

$$
\begin{gathered}
\lim _{u \rightarrow 0^{+}} u^{-1} G(u)=0, \lim _{u \rightarrow \infty} u^{-1} G(u)=\infty, \\
\lim _{u \rightarrow \infty} u^{-5} G(u)=0,
\end{gathered}
$$

Proof. The regularity of $G$ follows from Lemma 8.1, since $G(u)=w(0,0, u)$. We have

$$
G(u)=4 \pi \sqrt{2} u^{3 / 2} \int_{0}^{1} \phi(-u \tau, 0) \sqrt{1-\tau} d \tau .
$$

By (6.7), $\forall \epsilon>0, \exists \delta>0$ such that for $u<\delta$ we have

$$
u^{-1} G(u)<4 \pi \sqrt{2} u^{1 / 2} \int_{0}^{1} \epsilon(u \tau)^{-1 / 2} \sqrt{1-\tau} d \tau=C \epsilon .
$$

This proves the first limit in (6.23). Similarly by (6.8), $\forall \epsilon>0, \exists \delta>0$ such that for $u>1 / \delta$ we have

$$
u^{-1} G(u)>C u^{1 / 2} \int_{1 / 2}^{1} \frac{1}{\epsilon}(u \tau)^{-1 / 2} \sqrt{1-\tau} d \tau=\frac{C}{\epsilon},
$$

which proves the second limit in (6.23). Finally, by (6.7) and (6.9), $\forall \epsilon>0, \exists \delta>0$ such that for $K>1 / \delta$ we have

$$
\begin{aligned}
u^{-5} G(u) & \leq C u^{-7 / 2} \int_{0}^{K / u} \phi(-u \tau, 0) \sqrt{1-\tau} d \tau+C u^{-7 / 2} \int_{K / u}^{1} \phi(-u \tau, 0) \sqrt{1-\tau} d \tau \\
& \leq C u^{-7 / 2} \sup _{-K \leq t \leq 0}|t|^{1 / 2} \phi(t, 0) \int_{0}^{K / u}(u \tau)^{-1 / 2} \sqrt{1-\tau} d \tau+C u^{-7 / 2} \int_{K / u}^{1} \epsilon(u \tau)^{7 / 2} \sqrt{1-\tau} d \tau .
\end{aligned}
$$

Now choose $u$ sufficiently large to make the above less than $C \epsilon$. This proves (6.24).

As in Lemma 3.2 conditions (6.23) and (6.24) are precisely what is needed for the existence of a positive radial solution $U_{0}$ to (6.22) with zero boundary condition defined on $B_{1}$ and satisfying $U_{0}^{\prime}(|x|)<0$ for $0<|x| \leq 1$. We extend $U_{0}(|x|)$ to be radial and harmonic in $\{|x|>1\}$ such that $U_{0} \in C^{1}\left(\mathbb{R}^{3}\right)$. That is, $U_{0}(|x|)=(1-1 /|x|) U_{0}^{\prime}(1)$ for $|x|>1$. This completes the proof of Proposition 6.1 since the regularity assertion follows easily.

\section{VLASOV MODEL: ANALYSIS OF THE LINEARIZED OPERATOR}

\subsection{Linearization.}

Theorem 7.1. The operator $\mathcal{F}: B_{\epsilon}(X) \times \mathbb{R} \rightarrow X$ with $\epsilon>0$ sufficiently small is continuously Fréchet differentiable with $\frac{\partial \mathcal{F}}{\partial \zeta}$ given by (8.13) and (8.14) below.

We postpone the proof of this theorem until Section 8 . The operator $\mathcal{F}(\zeta, \kappa)$ is re-described in (8.1)- (8.3). We now compute $\mathcal{L}=\frac{\partial \mathcal{F}}{\partial \zeta}(0,0)$ using (8.13) and (8.14). Noticing

$$
\mathcal{M}(0,0)=D\left(0, U_{0}\right)=M, \quad w(0,0, u)=G(u), \quad \rho_{0}=G\left(U_{0}\right),
$$

which are merely definitions, we soon get

$$
\begin{aligned}
& {[\mathcal{L} \xi](x)=\left[\frac{\partial \mathcal{F}}{\partial \zeta}(0,0) \xi\right](x) } \\
= & -\int_{B_{1}} \rho_{0}^{\prime}(y) \frac{\xi(y)}{|y|}\left(\frac{1}{|x-y|}-\frac{1}{|y|}\right) d y-\left\{\int_{B_{1}} \rho_{0}(|y|) \frac{x-y}{|x-y|^{3}} d y\right\} \cdot \frac{\xi(x) x}{|x|^{2}} \\
& +\frac{1}{M}\left\{\int_{B_{1}} \rho_{0}^{\prime}(y) \frac{\xi(y)}{|y|} d y\right\}\left\{\int_{B_{1}} \rho_{0}(y)\left(\frac{1}{|x-y|}-\frac{1}{|y|}\right) d y\right\} .
\end{aligned}
$$


In the above, prime $\left({ }^{\prime}\right)$ means radial derivative $\partial_{r}$, where $r=|x|$. Now using again the calculation (4.5) and the equivalent expression of $\mathcal{F}(0,0)=0$ :

$$
U_{0}(x)-U_{0}(0)=\int_{B_{1}} \rho_{0}(y)\left(\frac{1}{|x-y|}-\frac{1}{|y|}\right) d y,
$$

we get

$$
\begin{aligned}
& {[L \xi](x)=\left[\frac{\partial \mathcal{F}}{\partial \zeta}(0,0) \xi\right](x) } \\
= & \frac{U_{0}^{\prime}(x)}{|x|} \xi(x)-\int_{B_{1}} \rho_{0}^{\prime}(y) \frac{\xi(y)}{|y|}\left(\frac{1}{|x-y|}-\frac{1}{|y|}\right) d y \\
& +\frac{1}{M}\left(U_{0}(x)-U_{0}(0)\right) \int_{B_{1}} \rho_{0}^{\prime}(y) \frac{\xi(y)}{|y|} d y .
\end{aligned}
$$

Comparing with the linearized operator in the Euler model, we observe that the first two terms in (4.8) and (7.2) are the same. The difference in the last term results from the different ways the mass balancing factor appears in the Euler model and the Vlasov model. In the Euler model, the mass factor appears inside the enthalpy function $h$, whereas in the Vlasov model, it appears directly in front of the last term in the equation. The difference in the last term results in some crucial alteration in the analysis of the radial part of the kernel of $\mathcal{L}$. In particular, for the Vlasov model, the triviality of the kernel can be established in general and does not require a condition on the total mass as in the Euler model.

7.2. Analysis of the Vlasov kernel. In this section we prove that $\mathcal{L}$ is an isomorphism on $X$.

Theorem 7.2. The linearized operator $\mathcal{L}$ is injective.

By (7.2), $\xi \in X$ belongs to the kernel of $\mathcal{L}$ if and only if the expression in (7.2) vanishes. Defining $\alpha(x)=\frac{U_{0}^{\prime}(x)}{|x|} \xi(x)$ for convenience, we have

$$
0=\alpha(x)-\int_{B_{1}} \frac{\rho_{0}^{\prime}(y)}{U_{0}^{\prime}(y)} \alpha(y)\left(\frac{1}{|x-y|}-\frac{1}{|y|}\right) d y+\frac{1}{M}\left(U_{0}(x)-U_{0}(0)\right) \int_{B_{1}} \frac{\rho_{0}^{\prime}(y)}{U_{0}^{\prime}(y)} \xi(y) d y .
$$

Our goal is to prove that $\alpha \equiv 0$. Note that $\alpha(0)=0$. Taking the Laplacian of both sides, we get

$$
\Delta \alpha+4 \pi \frac{\rho_{0}^{\prime}}{U_{0}^{\prime}} \alpha-\frac{1}{M}\left(\int_{B_{1}} \frac{\rho_{0}^{\prime}}{U_{0}^{\prime}} \alpha d y\right) 4 \pi \rho_{0}=0 .
$$

Lemma 7.1. No radial function belongs to the nullspace of the linearized operator $\mathcal{L}$.

Proof. This is the most delicate and novel part of the isomorphism proof. Let $\alpha=\alpha(|x|)$ be a radial function satisfying (7.3). Note that $\alpha^{\prime}(0)=0$, where the prime denotes the radial derivative. Integrating (7.4) over $|x|<1$, the second and third terms exactly cancel each other and so we get $\alpha^{\prime}(1)=0$. Summarizing, we have (7.4) together with

$$
\alpha(0)=\alpha^{\prime}(0)=\alpha^{\prime}(1)=0 .
$$

We want to prove that $\alpha \equiv 0$.

By (6.20) and (6.22), we have

$$
\frac{\rho_{0}^{\prime}}{U_{0}^{\prime}}=\frac{\left(G\left(U_{0}\right)\right)^{\prime}}{U_{0}^{\prime}}=G^{\prime}\left(U_{0}\right)
$$

on $B_{1}$. So we can rewrite (7.4) as

$$
\Delta \alpha+4 \pi G^{\prime}\left(U_{0}\right) \alpha-\frac{1}{M}\left(\int_{B_{1}} G^{\prime}\left(U_{0}\right) \alpha d y\right) 4 \pi G\left(U_{0}\right)=0 .
$$


This is the basic equation for $\alpha$ from which, together with (7.5), we want to prove that it vanishes. Note that, because of (7.5), if $\int_{B_{1}} G^{\prime}\left(U_{0}\right) \alpha d y=0$, then $\alpha=0$.

On the contrary let us assume that $\int_{B_{1}} G^{\prime}\left(U_{0}\right) \alpha d y \neq 0$ and define $\beta=\frac{M}{\int_{B_{1}} G^{\prime}\left(U_{0}\right) \alpha d y} \alpha$, so that

$$
\Delta \beta+4 \pi G^{\prime}\left(U_{0}\right) \beta-4 \pi G\left(U_{0}\right)=0
$$

and

$$
\beta(0)=\beta^{\prime}(0)=\beta^{\prime}(1)=0 .
$$

To show that $\beta=0$, we consider the family of solutions to the following variation of (6.22):

$$
v^{\prime \prime}+\frac{2}{r} v^{\prime}+4 \pi S G(v)=0, \quad v(0)=a, v^{\prime}(0)=0 .
$$

Here $S$ and $a$ are parameters, $r=|x|$. We denote the unique solution to (7.10) by $v(r ; a, S)$. By (6.22),$v\left(r ; U_{0}(0), 1\right)=U_{0}(r)$. Differentiating (7.10) with respect to $S$, and setting $S=1$, $a=U_{0}(0)$, we get

$$
\Delta v_{S}+4 \pi G^{\prime}\left(U_{0}\right) v_{S}+4 \pi G\left(U_{0}\right)=0, \quad v_{S}(0)=0, v_{S}^{\prime}(0)=0,
$$

where $v_{S}(r)=v_{S}\left(r ; U_{0}(0), 1\right), v_{a}(r)=v_{a}\left(r ; U_{0}(0), 1\right)$. The subscripts in $v_{S}$ and $v_{a}$ denote partial derivatives. Since (7.8) and (7.11) are essentially linear ODEs with the same vanishing initial conditions and homogeneous terms and opposite nonhomogeneous terms, we must have

$$
\beta=-v_{S}
$$

Note that a simple rescaling yields us $v(R r ; a, S)=v\left(r ; a, R^{2} S\right)$. Differentiating with respect to $R$ and setting $R=S=1, a=u_{0}(0)$, we get

$$
r v^{\prime}=2 v_{S} .
$$

Taking the $r$ derivative and setting $r=1$, we get

$$
2 v_{S}^{\prime}(1)=v^{\prime}(1)+v^{\prime \prime}(1)=v^{\prime}(1)-2 v^{\prime}(1)-G(v(1))=-v^{\prime}(1) .
$$

Here we have used (7.10), $v(1)=U_{0}(1)=0$ and $G(0)=0$. The condition $\beta^{\prime}(1)=0$ in (7.9) and the relation (7.12) imply $v_{S}^{\prime}(1)=0$, which implies $v^{\prime}(1)=0$ by (7.14). But the boundary condition is $v(1)=0$, which means that the initial data for $v(r)$ at $r=1$ vanish. Thus $v \equiv 0$, which contradicts $v=U_{0}>0$.

Proof of Theorem 7.2. It remains to consider the non-radial part of the kernel of $\mathcal{L}$. But the argument is identical to the Euler case, because the first two terms in (4.8) and (7.2) are the same. The only thing we used from the last term of (4.8) is that it is radial and hence is orthogonal to any non-radial spherical harmonic. This is still the case for the last term in (7.2).

Now we prove the compactness.

Lemma 7.2. $\mathcal{L}: X \rightarrow X$ has the form $\mathcal{L}=J+K$ where $J$ is an isomorphism, and $K$ is a compact operator.

Proof. Recall that in the definition of $\mathcal{L}$ given in (7.2), only the last term differs from the Euler case. Since the last term is a rank one operator, it is compact.

By the standard implicit function theorem, Theorem 6.1 follows by combining the preceding results, as in the Euler case. 


\section{VLASOV MOdEL: FrÉCHET DIFFERENTIABILITY}

In this section we prove the Fréchet differentiability of the map

$\begin{aligned} & \mathcal{F}(\zeta, \kappa)(x) \\ (8.1)= & -U_{0}(x)+U_{0}(0)+\frac{M}{\mathcal{M}(\zeta, \kappa)} \int_{B_{2}} w\left(\kappa, r(y), U_{0}\left(g_{\zeta}^{-1}(y)\right)\right)\left[\frac{1}{\left|g_{\zeta}(x)-y\right|}-\frac{1}{|y|}\right] d y\end{aligned}$

where

$$
\mathcal{M}(\zeta, \kappa)=\int_{B_{2}} w\left(\kappa, r(y), U_{0}\left(g_{\zeta}^{-1}(y)\right)\right) d y=D\left(\kappa, U_{0} \circ g_{\zeta}^{-1}\right)
$$

and

$$
w(\kappa, r, u)=2 \pi \int_{-u}^{0} \int_{-\sqrt{2(E+u)}}^{\sqrt{2(E+u)}} \phi(E, \kappa r s) d s d E .
$$

This is a different map from the one in the Euler model but the space $X$ will be the same. We wish to prove Theorem 7.1 which states that the operator $\mathcal{F}$ is continuously Fréchet differentiable on $B_{\epsilon}(X) \times \mathbb{R}$. We first state a lemma that describes the regularity of $w(\kappa, r, u)$.

Lemma 8.1. $w(\kappa, r, u)$ defined by (8.3) is in $C^{1}(\mathbb{R} \times[0, \infty) \times \mathbb{R}), \partial_{r} w \in C^{1}(\mathbb{R} \times[0, \infty) \times \mathbb{R})$, and for every bounded set $B \subset \mathbb{R} \times[0, \infty) \times \mathbb{R}$ there exists a constant $C>0$ with

$$
\begin{gathered}
\left|\partial_{r} w(\kappa, r, u)\right| \leq C r \\
\left|w\left(\kappa_{1}, r, u_{1}\right)-w\left(\kappa_{2}, r, u_{2}\right)\right| \leq C\left(\left|\kappa_{1}-\kappa_{2}\right| r+\left|u_{1}-u_{2}\right|\right), \\
\left|\partial_{u} w\left(\kappa_{1}, r, u_{1}\right)-\partial_{u} w\left(\kappa_{2}, r, u_{2}\right)\right| \leq C\left(\left|\kappa_{1}-\kappa_{2}\right|+\left|u_{1}-u_{2}\right|^{\nu}\right)
\end{gathered}
$$

for all $\left(\kappa_{1}, r, u_{1}\right),\left(\kappa_{2}, r, u_{2}\right) \in B$. Here $\nu=\frac{1}{2}-\mu$, with $\mu$ given as in (6.7).

Proof. The proof generalizes that of Lemma 2.1 in [17]. By assumption (6.7) we have $0 \leq$ $\phi(E, L) \leq C|E|^{-1 / 2}$ for bounded $L$ and $E$. Thus

$$
|w(\kappa, r, u)| \leq C_{1} \int_{-u}^{0} \sqrt{\frac{E+u}{|E|}} d E=C_{1} \int_{0}^{u} \sqrt{\frac{u-s}{s}} d s \leq C_{2} .
$$

In a similar way, (8.5) follows. Now

$$
\partial_{r} w(\kappa, r, u)=2 \pi \kappa \int_{-u}^{0} \int_{-\sqrt{2(E+u)}}^{\sqrt{2(E+u)}} \partial_{L} \phi(E, \kappa r s) s d s d E .
$$

Because $\partial_{L} \phi(E, 0)=0$, we have $\left|\partial_{L} \phi(E, \kappa r s)\right| \leq\left|\partial_{L}^{2} \phi(E, \theta \kappa r s)\right| \kappa r s$ for some $\theta \in(0,1)$. Furthermore $\left|\partial_{L}^{2} \phi(E, L)\right| \leq C|E|^{-1 / 2}$ for bounded $E$ and $L$ by (6.7). Thus (8.4) follows.

Finally we have

$$
\partial_{u} w(\kappa, r, u)=\pi \sqrt{2} \int_{-u}^{0}\{\phi(E, \kappa r \sqrt{2(E+u)})+\phi(E,-\kappa r \sqrt{2(E+u)})\} \frac{1}{\sqrt{E+u}} d E .
$$


Without loss of generality we assume $u_{1} \geq u_{2}>0$. By (6.7) we have

$$
\begin{aligned}
& \leq C\left[\sup _{|E|,|L| \leq B_{0}}|E|^{\mu}|\phi(E, L)|\right] \int_{-u_{1}}^{-u_{2}} \frac{|E|^{-\mu}}{\sqrt{E+u_{1}}} d E \\
&+C\left[\sup _{|E|,|L| \leq B_{0}}|E|^{1 / 2}\left|\partial_{L} \phi(E, L)\right|\right] \int_{-u_{2}}^{0} \frac{1}{\sqrt{|E|\left(E+u_{1}\right)}}\left[\sqrt{E+u_{1}}-\sqrt{E+u_{2}}\right] d E \\
&+C\left[\sup _{|E|,|L| \leq B_{0}}|E|^{\mu}|\phi(E, L)|\right] \int_{-u_{2}}^{0}|E|^{-\mu}\left[\frac{1}{\sqrt{E+u_{2}}}-\frac{1}{\sqrt{E+u_{1}}}\right] d E \\
& \leq C\left|u_{1}-u_{2}\right|^{\frac{1}{2}-\mu}+C\left|u_{1}-u_{2}\right|^{\frac{1}{2}}+C\left|u_{1}-u_{2}\right|^{\frac{1}{2}-\mu} .
\end{aligned}
$$

The last inequality is justified as follows. In the first integral in (8.7) we change variables $F=-E$ and then $F=t u_{2}$ and $u_{1}=s u_{2}$ with $1 \leq s \leq 2$ to obtain

$$
\begin{aligned}
& \int_{u_{2}}^{u_{1}} \frac{1}{F^{\mu}\left(u_{1}-F\right)^{\frac{1}{2}}} d F=u_{2}^{\frac{1}{2}-\mu} \int_{1}^{s} \frac{1}{t^{\mu}(s-t)^{\frac{1}{2}}} d t \leq u_{2}^{\frac{1}{2}-\mu} \int_{1}^{s} \frac{1}{(s-t)^{\frac{1}{2}}} d t \\
& =2 u_{2}^{\frac{1}{2}-\mu}(s-1)^{\frac{1}{2}} \leq 2\left[u_{2}(s-1)\right]^{\frac{1}{2}-\mu}=2\left(u_{1}-u_{2}\right)^{\frac{1}{2}-\mu} .
\end{aligned}
$$

On the other hand, if $s>2$, the same integral is bounded by

$$
u_{2}^{\frac{1}{2}-\mu} \int_{0}^{s} \frac{1}{t^{\mu}(s-t)^{\frac{1}{2}}} d t=C u_{2}^{\frac{1}{2}-\mu} s^{\frac{1}{2}-\mu} \leq C\left(u_{2} 2(s-1)\right)^{\frac{1}{2}-\mu}=C\left[2\left(u_{1}-u_{2}\right)\right]^{\frac{1}{2}-\mu} .
$$

The other terms in (8.7) can be estimated in a similar fashion, which leads to (8.6).

To show that $\mathcal{F}(\zeta, \kappa) \in X$, we estimate its spatial derivatives. As for the Euler case, we can differentiate (8.1) under the integral sign. Consequently, there exists $\epsilon>0$, such that for all $\zeta \in B_{\epsilon}(X)$,

$$
\partial_{i} \mathcal{F}(\zeta, \kappa)(x)=-\partial_{i} U_{0}(x)+\frac{M}{\mathcal{M}(\zeta, \kappa)} \int_{B_{2}} w\left(\kappa, r(y), U_{0}\left(g_{\zeta}^{-1}(y)\right)\right) \frac{-\left(g_{\zeta}(x)-y\right)}{\left|g_{\zeta}(x)-y\right|^{3}} d y \cdot \partial_{i} g_{\zeta}(x)
$$

Next we estimate the second term in (8.9).

$$
\left|w\left(\kappa, r(y), U_{0}\left(g_{\zeta}^{-1}(y)\right)\right)-w\left(\kappa, 0, U_{0}\left(g_{\zeta}^{-1}(0)\right)\right)\right| \leq C\left(r(y)+\left|U_{0}\left(g_{\zeta}^{-1}(y)\right)-U_{0}\left(g_{\zeta}^{-1}(0)\right)\right|\right)
$$

$$
\leq C\left(r(y)+\left\|\nabla U_{0}\right\|_{\infty}\left|g_{\zeta}^{-1}(y)\right|\right) \leq C\left(1+\|\zeta\|_{X}\right)|y|
$$

Therefore if $|\kappa| \leq \bar{\kappa}$, and $\zeta \in B_{\epsilon}(X)$ for some $\epsilon$ small enough, there is a constant $C>0$ such that

$$
\|\mathcal{F}(\zeta, \kappa)\|_{X} \leq C\left(1+\|\zeta\|_{X}\right)
$$

Next we compute the formal derivative. As in the Euler model, we define $F(x, s)=$ $\mathcal{F}(\zeta+s \xi, \kappa)(x)$ and we define the formal derivative as

$$
\left[\mathcal{F}^{\prime}(\zeta, \kappa) \xi\right](x)=\partial_{s} F(x, 0)=\left.\partial_{s}\right|_{s=0} \mathcal{F}(\zeta+s \xi, \kappa)(x)
$$


Lemma 8.2. The formal derivative $\left[\mathcal{F}^{\prime}(\zeta, \kappa) \xi\right](x)$ exists and equals

$$
\begin{aligned}
& -\frac{M}{\mathcal{M}(\zeta, \kappa)} \int_{B_{2}} \partial_{u} w\left(\kappa, r(y), U_{0}\left(g_{\zeta}^{-1}(y)\right)\right) \\
& \quad \nabla U_{0}\left(g_{\zeta}^{-1}(y)\right) D\left(g_{\zeta}^{-1}\right)(y) \xi\left(g_{\zeta}^{-1}(y)\right) \frac{g_{\zeta}^{-1}(y)}{\left|g_{\zeta}^{-1}(y)\right|^{2}}\left[\frac{1}{\left|g_{\zeta}(x)-y\right|}-\frac{1}{|y|}\right] d y \\
& -\frac{M}{\mathcal{M}(\zeta, \kappa)} \int_{B_{2}} w\left(\kappa, r(y), U_{0}\left(g_{\zeta}^{-1}(y)\right)\right) \frac{g_{\zeta}(x)-y}{\left|g_{\zeta}(x)-y\right|^{3}} d y \cdot \frac{x \xi(x)}{|x|^{2}} \\
& -\frac{M\left[\mathcal{M}^{\prime}(\zeta, \kappa) \xi\right]}{\mathcal{M}^{2}(\kappa, \zeta)} \int_{B_{2}} w\left(\kappa, r(y), U_{0}\left(g_{\zeta}^{-1}(y)\right)\right)\left[\frac{1}{\left|g_{\zeta}(x)-y\right|}-\frac{1}{|y|}\right] d y,
\end{aligned}
$$

where

$$
\mathcal{M}^{\prime}(\zeta, \kappa) \xi=-\int_{B_{2}} \partial_{u} w\left(\kappa, r(y), U_{0}\left(g_{\zeta}^{-1}(y)\right)\right) \nabla U_{0}\left(g_{\zeta}^{-1}(y)\right) D g_{\zeta}^{-1}(y) \xi\left(g_{\zeta}^{-1}(y)\right) \frac{g_{\zeta}^{-1}(y)}{\left|g_{\zeta}^{-1}(y)\right|^{2}} d y
$$

Proof. We use the cutoff function method as before. As is the case for estimates (5.29) and (5.31), the key estimates

$$
\begin{gathered}
\left|w\left(\kappa, r(y), U_{0}\left(g_{\zeta+s \xi}^{-1}(y)\right)\right) \frac{1}{\left|g_{\zeta+s \xi}(x)-y\right|}\right| \leq \frac{C}{\left|g_{\zeta+s \xi}(x)-y\right|}, \\
\left|\partial_{s}\left[w\left(\kappa, r(y), U_{0}\left(g_{\zeta+s \xi}^{-1}(y)\right)\right) \frac{1}{\left|g_{\zeta+s \xi}(x)-y\right|}\right]\right| \leq \frac{C}{\left|g_{\zeta+s \xi}(x)-y\right|^{2}}
\end{gathered}
$$

are easily proven.

Lemma 8.3. The spatial derivatives of $\mathcal{F}^{\prime}(\zeta, \kappa) \xi$ are

$$
\begin{aligned}
& \partial_{i}\left[\mathcal{F}^{\prime}(\zeta, \kappa) \xi\right](x) \\
= & \frac{M}{\mathcal{M}(\zeta, \kappa)} \int_{B_{2}} \partial_{u} w\left(\kappa, r(y), U_{0}\left(g_{\zeta}^{-1}(y)\right)\right) \\
& \nabla U_{0}\left(g_{\zeta}^{-1}(y)\right) D g_{\zeta}^{-1}(y) \xi\left(g_{\zeta}^{-1}(y)\right) \frac{g_{\zeta}^{-1}(y)}{\left|g_{\zeta}^{-1}(y)\right|^{2}} \frac{g_{\zeta}(x)-y}{\left|g_{\zeta}(x)-y\right|^{3}} d y \cdot \partial_{i} g_{\zeta}(x) \\
& +\sum_{j} \frac{M}{\mathcal{M}(\zeta, \kappa)} \int_{B_{2}}\left[w_{r}\left(\kappa, r(y), U_{0}\left(g_{\zeta}^{-1}(y)\right)\right) \partial_{j} r(y)\right. \\
& \left.+w_{u}\left(\kappa, r(y), U_{0}\left(g_{\zeta}^{-1}(y)\right)\right) \cdot \nabla U_{0}\left(g_{\zeta}^{-1}(y)\right) \partial_{j} g_{\zeta}^{-1}(y)\right] \frac{g_{\zeta}(x)-y}{\left|g_{\zeta}(x)-y\right|^{3}} d y \cdot \partial_{i} g_{\zeta}(x) \frac{x_{j} \xi(x)}{|x|^{2}} \\
& -\frac{M}{\mathcal{M}(\zeta, \kappa)} \int_{B_{2}} w\left(\kappa, r(y), U_{0}\left(g_{\zeta}^{-1}(y)\right)\right) \frac{g_{\zeta}(x)-y}{\left|g_{\zeta}(x)-y\right|^{3}} d y \cdot \partial_{i}\left(\frac{x \xi(x)}{|x|^{2}}\right) \\
& +\frac{M\left[\mathcal{M}^{\prime}(\zeta, \kappa) \xi\right]}{\mathcal{M}^{2}(\zeta, \kappa)} \int_{B_{2}} w\left(\kappa, r(y), U_{0}\left(g_{\zeta}^{-1}(y)\right) \frac{g_{\zeta}(x)-y}{\left|g_{\zeta}(x)-y\right|^{3}} d y \cdot \partial_{i} g_{\zeta}(x) .\right.
\end{aligned}
$$

Proof. We apply the cutoff function method to (8.13). The second term of (8.13) is written as

$$
\sum_{j} \int_{B_{2}} w\left(\kappa, r(y), U_{0}\left(g_{\zeta}^{-1}(y)\right)\right) \partial_{y_{j}}\left(\frac{1}{\left|g_{\zeta}(x)-y\right|}\right) d y \frac{x_{j} \xi(x)}{|x|^{2}}
$$

and then integrated by parts to get

$$
-\sum_{j} \int_{B_{2}} \partial_{y_{j}}\left(w\left(\kappa, r(y), U_{0}\left(g_{\zeta}^{-1}(y)\right)\right)\right) \frac{1}{\left|g_{\zeta}(x)-y\right|} d y \frac{x_{j} \xi(x)}{|x|^{2}} .
$$

Details are omitted. 
Lemma 8.4. The mixed partials of $F$ are

$$
\partial_{s} \partial_{i} F(x, s)=\partial_{i} \partial_{s} F(x, s)=\partial_{i}\left[\mathcal{F}^{\prime}(\zeta+s \xi, \kappa)\right](x) .
$$

Proof. The second equality in (8.20) is the content of Lemma 8.3. To get the first equality, we note that by (8.9), we have

$\partial_{i} F(x, s)=-\partial_{i} U_{0}(x)$

$$
+\frac{M}{\mathcal{M}(\zeta+s \xi, \kappa)} \int_{B_{2}} w\left(\kappa, r(y), U_{0}\left(g_{\zeta+s \xi}^{-1}(y)\right)\right) \frac{-\left(g_{\zeta+s \xi}(x)-y\right)}{\left|g_{\zeta+s \xi}(x)-y\right|^{3}} d y \cdot \partial_{i} g_{\zeta+s \xi}(x) .
$$

To calculate the $s$ derivative of the integral in (8.21), we apply the cutoff function to get

$$
\int_{B_{2}} w\left(\kappa, r(y), U_{0}\left(g_{\zeta+s \xi}^{-1}(y)\right)\right) \frac{-\left(g_{\zeta+s \xi}(x)-y\right)}{\left|g_{\zeta+s \xi}(x)-y\right|^{3}}\left[1-\chi\left(\frac{\left|g_{\zeta+s \xi}(x)-y\right|}{\epsilon}\right)\right] d y
$$

and compute the derivative as we did for (5.44).

Lemma 8.5. If $|\kappa| \leq \bar{\kappa}$, and $\zeta \in B_{\epsilon}(X)$ for some $\epsilon$ small enough, there is a constant $C>0$ such that

$$
\left\|\mathcal{F}^{\prime}(\zeta, \kappa) \xi\right\|_{X} \leq C \|_{X}
$$

Proof. We apply Lemma 3.6 to (8.17). To work out the first term, we just need

$$
\partial_{u} w\left(\kappa, r(y), U_{0}\left(g_{\zeta}^{-1}(y)\right)\right) \nabla U_{0}\left(g_{\zeta}^{-1}(y)\right) D\left(g_{\zeta}^{-1}\right)(y) \xi\left(g_{\zeta}^{-1}(y)\right) \frac{g_{\zeta}^{-1}(y)}{\left|g_{\zeta}^{-1}(y)\right|^{2}}
$$

to be bounded by $C\|\xi\|_{X}|y|$, which is similar to our treatment of the first term of (5.35). This bound is indeed achieved by the properties of $w$. Since the second term in (8.17) has a factor $\frac{x_{j} \xi(x)}{|x|^{2}}$, which is already bounded by $C\|\xi\|_{X}|x|$, we just need

$$
w_{r}\left(\kappa, r(y), U_{0}\left(g_{\zeta}^{-1}(y)\right)\right) \partial_{j} r(y)+w_{u}\left(\kappa, r(y), U_{0}\left(g_{\zeta}^{-1}(y)\right)\right) \cdot \nabla U_{0}\left(g_{\zeta}^{-1}(y)\right) \partial_{j} g_{\zeta}^{-1}(y)
$$

to be bounded, which is again true. For the third term in (8.17), we need

$$
\left|w\left(\kappa, r(y), U_{0}\left(g_{\zeta}^{-1}(y)\right)\right)-w\left(\kappa, 0, U_{0}(0)\right)\right| \leq C|y|,
$$

which is also true since $w$ is $C^{1}$. The last term is similar.

Lemma 8.6. If $|\kappa| \leq \bar{\kappa}$, and $\zeta \in B_{\epsilon}(X)$ for some $\epsilon$ small enough, there is a constant $C>0$ such that

$$
\left\|\left(\mathcal{F}^{\prime}\left(\zeta_{1}, \kappa\right)-\mathcal{F}^{\prime}\left(\zeta_{2}, \kappa\right)\right) \xi\right\|_{X} \leq C\left\|\zeta_{1}-\zeta_{2}\right\|_{X}^{\nu}\|\xi\|_{X}
$$

Proof. We compute the spatial derivatives of $\left(\mathcal{F}^{\prime}\left(\zeta_{1}, \kappa\right)-\mathcal{F}^{\prime}\left(\zeta_{2}, \kappa\right)\right) \xi$ using (8.17) and we estimate as in Lemma 5.6. We first note that $\mid \mathcal{M}\left(\zeta_{1}, \kappa\right)-\mathcal{M}\left(\zeta_{2}, \kappa \mid \leq C\left\|\zeta_{1}-\zeta_{2}\right\|_{X}\right.$ because

$$
\left|w\left(\kappa, r(y), U_{0}\left(g_{\zeta_{1}}^{-1}(y)\right)\right)-w\left(\kappa, r(y), U_{0}\left(g_{\zeta_{2}}^{-1}(y)\right)\right)\right| \leq C\left\|\zeta_{1}-\zeta_{2}\right\|_{X} .
$$

For the first term in (8.17), the key estimate is

$$
\left|\partial_{u} w\left(\kappa, r(y), U_{0}\left(g_{\zeta_{1}}^{-1}(y)\right)\right)-\partial_{u} w\left(\kappa, r(y), U_{0}\left(g_{\zeta_{2}}^{-1}(y)\right)\right)\right| \leq C\left\|\zeta_{1}-\zeta_{2}\right\|_{X}^{\nu} .
$$

For the second term in (8.17), the key estimate is

$$
\left|\partial_{r} w\left(\kappa, r(y), U_{0}\left(g_{\zeta_{1}}^{-1}(y)\right)\right)-\partial_{r} w\left(\kappa, r(y), U_{0}\left(g_{\zeta_{2}}^{-1}(y)\right)\right)\right| \leq C\left\|\zeta_{1}-\zeta_{2}\right\|_{X}^{\nu},
$$

which is a consequence of the fact that

$$
\left|\partial_{r} w(\kappa, r, u)-\partial_{r} w\left(\kappa, r, u^{\prime}\right)\right| \leq C r\left|u-u^{\prime}\right|^{1 / 2}
$$

locally uniformly. For the third term in (8.17), the key estimate is

$$
\left|w\left(\kappa, r(y), U_{0}\left(g_{\zeta_{1}}^{-1}(y)\right)\right)-w\left(\kappa, r(y), U_{0}\left(g_{\zeta_{2}}^{-1}(y)\right)\right)\right| \leq C\left\|\zeta_{1}-\zeta_{2}\right\|_{X}|y| .
$$


For the fourth term, we need to estimate $\left(\mathcal{M}^{\prime}\left(\zeta_{1}, \kappa\right)-\mathcal{M}^{\prime}\left(\zeta_{2}, \kappa\right)\right) \xi$ using (8.14). The key estimate again is (8.29).

Lemma 8.7. If $|\kappa| \leq \bar{\kappa}$, and $\zeta \in B_{\epsilon}(X)$ for some $\epsilon$ small enough. Let $\xi$ be such that $\zeta+s \xi \in B_{\epsilon}(X)$ for all $s \in[-1,1]$. Then

$$
\left\|\mathcal{F}(\zeta+\xi, \kappa)-\mathcal{F}(\zeta, \kappa)-\mathcal{F}^{\prime}(\zeta, \kappa) \xi\right\|_{X} \leq C\|\xi\|_{X}^{1+\nu}
$$

Proof. The proof is basically identical to that of Lemma 5.7

This completes the proof of Theorem 7.1 .

\section{REFERENCES}

[1] Ambrosetti, A., And Rabinowitz, P. H. Dual variational methods in critical point theory and applications. Journal of functional Analysis 14, 4 (1973), 349-381.

[2] Auchmuty, G. The global branching of rotating stars. Archive for Rational Mechanics and Analysis 114, 2 (1991), 179-193.

[3] Auchmuty, J., And Beals, R. Variational solutions of some nonlinear free boundary problems. Archive for Rational Mechanics and Analysis 43, 4 (1971), 255-271.

[4] Caffarelli, L. A., and Friedman, A. The shape of axisymmetric rotating fluid. Journal of Functional Analysis 35, 1 (1980), 109-142.

[5] Chanillo, S., AND Li, Y. Y. On diameters of uniformly rotating stars. Communications in Mathematical Physics 166, 2 (1994), 417-430.

[6] De Figueiredo, D. G., Lions, P., and Nussbaum, R. A priori estimates and existence of positive solutions of semilinear elliptic equations. In Djairo G. de Figueiredo-Selected Papers. Springer, 1982, pp. $133-155$.

[7] Evans, L. C. Partial differential equations, vol. 19 of Graduate Studies in Mathematics. American Mathematical Society, Providence, RI,, 2010.

[8] Friedman, A., And Turkington, B. Existence and dimensions of a rotating white dwarf. Journal of Differential Equations 42, 3 (1981), 414-437.

[9] Gidas, B., Ni, W.-M., And Nirenberg, L. Symmetry and related properties via the maximum principle. Communications in Mathematical Physics 68, 3 (1979), 209-243.

[10] Heilig, U. On Lichtenstein's analysis of rotating Newtonian stars. In Annales de l'IHP Physique théorique (1994), vol. 60, pp. 457-487.

[11] JANG, J., AND MAKINO, T. On slowly rotating axisymmetric solutions of the Euler-Poisson equations. arXiv preprint arXiv:1611.02812 (2016).

[12] Jardetzky, W. S. Theories of figures of celestial bodies. Courier Corporation, 2013.

[13] LI, Y. On uniformly rotating stars. Archive for rational mechanics and analysis 115, 4 (1991), 367-393.

[14] Lichtenstein, L. Untersuchungen über die Gleichgewichtsfiguren rotierender Flüssigkeiten, deren Teilchen einander nach dem Newtonschen Gesetze anziehen. Mathematische Zeitschrift 36, 1 (1933), 481-562.

[15] Luo, T., ANd Smoller, J. Existence and non-linear stability of rotating star solutions of the compressible Euler-Poisson equations. Archive for Rational Mechanics and Analysis 191, 3 (2009), 447-496.

[16] Ni, W.-M., And Nussbaum, R. D. Uniqueness and nonuniqueness for positive radial solutions of $\Delta u+$ $f(u, r)=0$. Communications on Pure and Applied Mathematics 38, 1 (1985), 67-108.

[17] Rein, G. Stationary and static stellar dynamic models with axial symmetry. Nonlinear Analysis: Theory, Methods 83 Applications 41 (2000), 313-344.

[18] Wu, Y. On rotating star solutions to the non-isentropic Euler-Poisson equations. Journal of Differential Equations 259, 12 (2015), 7161-7198.

[19] Wu, Y. Existence of rotating planet solutions to the Euler-Poisson equations with an inner hard core. Archive for Rational Mechanics and Analysis 219, 1 (2016), 1-26.

Department of Mathematics, Brown University, Providence, Ri 02912

Department of Mathematics, Brown University, Providence, Ri 02912 